\title{
From Monte Carlo Integration to Lattice Quantum Chromo Dynamics An introduction
}

\author{
Massimo Di Pierro \\ Fermilab, Batavia, IL 60510, USA \\ Email: mdp@fnal.gov
}

September 1, 2000

\begin{abstract}
Lectures on Lattice Field Theory and Lattice QCD given at the Graduate Students Association (GSA) Summer School (Fermilab).

In these lectures we provide a short introduction to the Monte Carlo integration method and its applications. We show how the origin of ultraviolet divergences if Field Theories is in the undefined formal product of distributions and how one can define the Path Integral in terms of regularized distributions in order to cancel these divergences. This technique provides the only non perturbative regularization procedure of continuum Field Theories and, at the same time, provides a practical method to compute correlation (Green) functions (using Monte Carlo integration for the regularized path integrals). We then apply these tools to formulate QCD on a lattice. Some of the examples are accompanied by complete computer programs.
\end{abstract}

Freely download libraries and examples from:

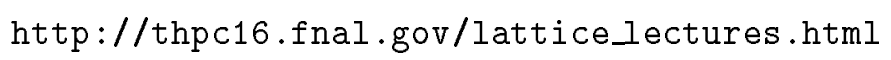




\section{Introduction}

Two are the main tasks a physicist has to confront with:

- [Induction]: given the symmetries of the measured observablesTbuild the underlying theory (i.e. write down an action).

- [Deduction]: given the action $\Gamma \mathcal{S} \Gamma$ compute correlation functions and $\Gamma$ from them $\Gamma$ physical observables (to test the theory and to make predictions).

In this notes we will focus the second task.

In the first section we wil see how the perturbative expansion of the Path Integral does not provide a satisfactory definition of the latter and a nonpertubartive regularization is necessary in order to define it properly.

For us the word "non-perturbative" means "exact up to a given precison that can be arbitrarily small".

In the second section we will show how to compute numerically $K$ dimensional integrals (for large integer $K$ ) using Monte Carlo techniques.

In the third section we will define the regularized Path Integral in terms of regularized distributions. This is equivalent to discretize the the spacetime on which the quantum fields are defined. The lattice spacing $a$ will play the role of an ultraviolet cut-off. We will then define the continuum Path Integral in terms of $K$-dimensional integrals in the limit $a \rightarrow 0$. We will show how this limit is the origin of ultraviolet divergences and how one can renormalize the theory by giving an $a$ dependence to the coupling constants that appear in the action.

Finally in the third section we will see how one can approximate a continuum Path Integral of QCD with a $K$-dimensional integral (for a finite $K$ ) and compute it numerically using Monte Carlo integration. We will discuss the sources of numerical errors and we will present $\Gamma$ as an example Cone full Lattice QCD application (the computation of $f_{B} \sqrt{m_{B}}$ ).

Some more examples of typical Lattice QCD computations are given in the Appendix.

The emphasis in these lectures will be given to three aspects that we consider crucial and make of lattice a privileged tool in respect to other model independent methods:

- The lattice regularization provides the only non-perturbative definition of Path Integral and $\Gamma$ therefore of Field Theories. 
- It is possible to quantify with precision the error committed in the numerical approximation of the integrals.

- It is $\Gamma$ in principle possible to reduce arbitrarily this error by approaching the continuum limit (reducing the lattice spacing) and increasing the statistical samples in the Monte Carlo integration.

This lectures are intended to be an introductory tutorial and they are not meant to be complete and/or exhaustive on the subject of lattice QCD.

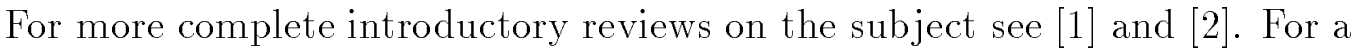
more complete and formal review see [i3i]

I wish to thank G. ChiodiniГB. DobrescuГE. EichtenГJ. JugeГ A. Kronfeld $\Gamma$ P. Mackenzie and J. Simone for helpful comments and suggestions regarding these notes.

This work was performed at FermilabГa U.S. Department of Energy Lab (operated by the University Research Association $\Gamma$ Inc.) $\Gamma$ under contract DEAC02-76CHO3000. 


\section{Contents}

$i^{-}$Corrélation functions, masses and matrix elements 1

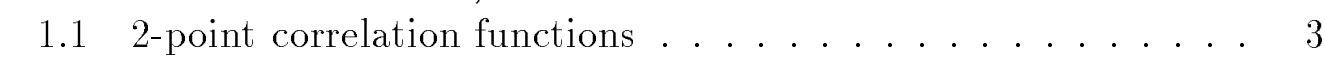

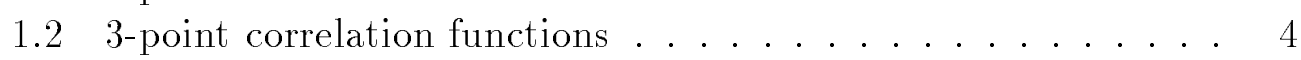

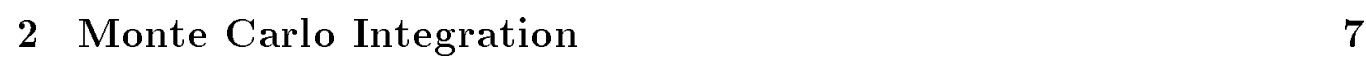

2.1 Riemann integrable functions . . . . . . . . . . . 7

Basic Monte-Carlo.................. 9

2.3 Metropolis Monte Carlo . . . . . . . . . . . . . . 10

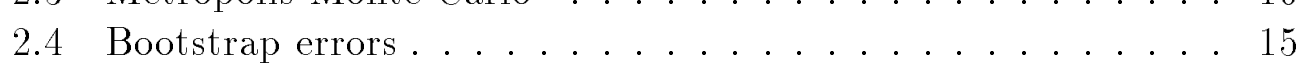

3. Definition of Path Integrals 18

3.1 Toy example: Regutarizing distributions . . . . . . . 19

Defining the Path Integrat . . . . . . . . . . . 22

Improving the convergence . . . . . . . . . . . . . . 26

Tattice regularization and momentum cut-off. . . . . . . . . . . 27

Remarkson the physics of Effective Theories . . . . . . . . 28

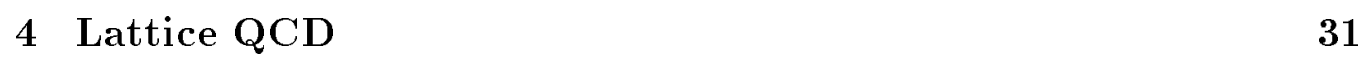

B. Basic degrees of freedom and action . . . . . . . . . 31

4.2 Simulation aspects and quenching . . . . . . . . . . . 36

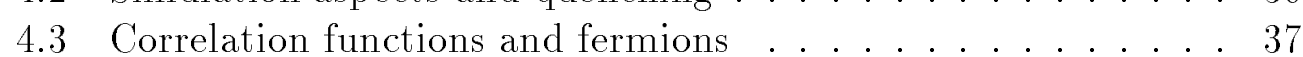

A.

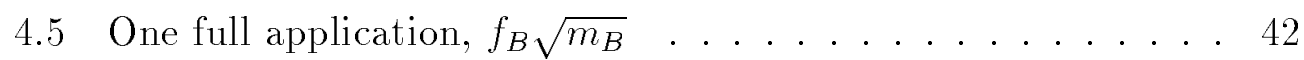

A' Euclidean Space-Time in $d=4$ dimensioñ 46

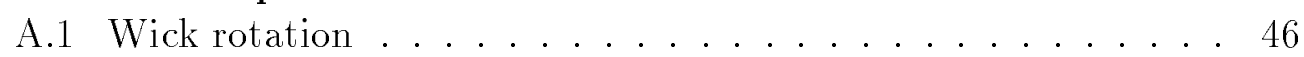

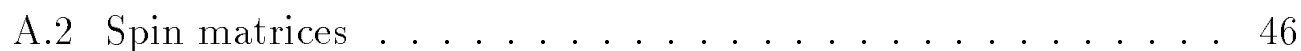

A Lattice discrete symmetries . . . . . . . . . . . . . . . . . . . . . . . . . . . 48

A.4 Theorems a bout correlation functions . . . . . . . . 48

B-Example programsi 49

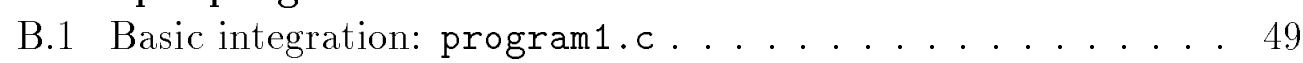

B. Monte Carlo integration: program2.c. . . . . . . . . 50

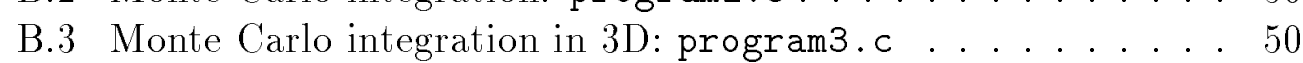

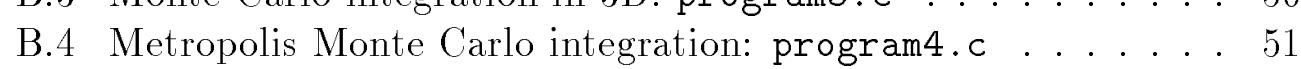

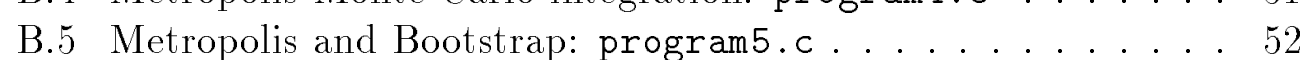

B. 


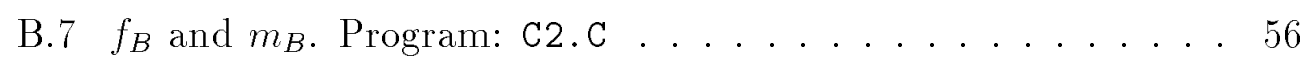

'Ć 


\section{Correlation functions, masses and matrix elements}

He who loves practice without theory is like the sailor who boards ship without a rudder and compass and never knows where he may cast

(Leonardo da Vinci)

The symbol of Path Integral [A]

$$
\left\langle 0\left|T\left\{\phi\left(x_{1}\right) \ldots \phi\left(x_{n}\right)\right\}\right| 0\right\rangle \stackrel{\text { def }}{=} \int[\mathrm{d} \phi] \phi\left(x_{1}\right) \ldots \phi\left(x_{n}\right) e^{-\mathcal{S}_{\mathrm{E}}[\phi]}
$$

provides a definition of the most general Euclidean correlation function (the left hand side) in terms of an infinite-dimensional integral (the path integral at the right hand side). $\mathcal{S}_{\mathrm{E}}[\phi]$ is the Euclidean action of the system and $\phi(x)$ represents the degrees of freedom of the system as function of the space-time Euclidean coordinates.

Without loss of generality we will only deal with Path Integrals in the Euclidean space since Minkowskian correlation functions can be obtained by analytic continuation of the Euclidean ones. In particular we are interested in extracting particle masses and matrix elements which do not have an explicit time dependence. Therefore $u p$ to a phase $\mathrm{Tthey}$ are the same in the Minkowskian and in the Euclidean formulation of the theory.

In the case of the $\phi^{4}$ scalar model the perturbative expansion Tafter renormalization $\Gamma$ is always convergent therefore it provides us with a definition of the Path Integral. Unfortunately...

In general, the perturbation expansion does not provide a satisfactory definition of the Path Integral.

Let's consider Tfor exampleГthe case of QCD. The perturbative expansion of Path Integrals of QCD is only convergent at high energy $(p>>200 \mathrm{MeV}) \Gamma$ 
i.e. at short distance $(a \equiv 1 / p<<1 \mathrm{fm})$. In fact the expansion parameter $\alpha_{s}=\frac{g(a)^{2}}{4 \pi}$ is scale dependent and it becomes big at low energy i.e. large

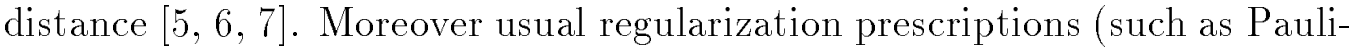
Villars and dimensional regularization) $\Gamma$ which are necessary to give sense to the Path IntegralГare only defined in a perturbative way.

One could argue that $\Gamma$ even if the perturbative expansion of eq.(否) is only valid at high energy continue the correlation functions from high to low energy and make somehow sense out of them. This is not correct!

First $\Gamma$ the perturbative series is an "asymptotic series" and it does not necessarily converge. Second (the most important point) The perturbative expansion is performed around the classical minimum of the action. The Euclidean action of QCD has many different minima that correspond to multiinstanton configurations. These are tunneling transitions between different vacua of QCD. These effects are not taken into account in the perturbative expansion but Tnevertheless They play a crucial role at low energy. They are believed to be responsible for the phenomenon of confinement [is].

The lattice discretization of the space-time provides us with the only non-perturbative regularization of the Path Integral and, therefore, with an EXACT definition of the latter.

For a small and finite cut-off (the lattice spacing a) the regularized theory can be seen as an effective theory of the continuum one (corresponding to the limit $a \rightarrow 0$ ). Such an effective theory is finite and free of ultraviolet divergences therefore it can be simulated numerically!

Moreover, in the case of QCD and gauge theories in general, lattice regularization has the nice feature to preserve gauge invariance even for every finite a.

We now introduce two examples in which we require a non-perturbative definition of the Path Integral to compute some important phenomenological quantities. 


\section{$1.1 \quad 2$-point correlation functions}

QCD is the theory of strong interactions Thence it must predict the masses of mesons and baryons from first principles. Let's consider hereГfor exampleГa $\mathrm{B}$ meson.

The following current

$$
J^{\mu}(x)=\bar{h}(x) \gamma^{\mu} \gamma^{5} q(x)
$$

(where $x_{\mu}=\left(x_{0} \equiv t_{x}, x_{1}, x_{2}, x_{3}\right) \Gamma t_{x}$ is the Wick rotated time and $h(q)$ is the fermionic field representing the heavy (light) quark) has the same flavor quantum numbers of a $\mathrm{B}$ meson. If we apply $J^{0}$ to the vacuum it must create some linear combination of a static B meson and its excited states

$$
J^{0}|0\rangle=\varepsilon_{0}|B\rangle+\varepsilon_{1}\left|B^{(1)}\right\rangle+\varepsilon_{2}\left|B^{(2)}\right\rangle+\ldots
$$

(in general there is a continuum of states but $\Gamma$ for notation $\Gamma$ we write them here as a sum.) From now on all our states are normalized as $\left\langle B^{(n)} \mid B^{(n)}\right\rangle=2 m_{B^{(n)}}$.

We define the following (zero momentum Fourier transform of the) two point correlation function

$$
\begin{aligned}
C_{2}\left(t_{x}\right) & =\int \mathrm{d}^{3} \mathrm{x}\left\langle 0\left|J^{0}(x) J^{0 \dagger}(0)\right| 0\right\rangle \\
& =\int\left[\mathrm{d} A_{\mu}\right]\left[\mathrm{d} q_{i}\right]\left[\mathrm{d} \bar{q}_{i}\right]\left(\int \mathrm{d}^{3} \mathrm{x} J^{0}(x) J^{0 \dagger}(0)\right) e^{-\mathcal{S}_{\mathrm{E}}^{\text {QCD }}}
\end{aligned}
$$

One can insert in the correlator a complete set of states...

$$
\begin{aligned}
\int \mathrm{d}^{3} \mathrm{x}\left\langle 0\left|J^{0}(x) J^{0 \dagger}(0)\right| 0\right\rangle & =\sum_{n} \int \mathrm{d}^{3} \mathbf{x}\left\langle 0\left|J^{0}(x) \frac{\left|B^{(n)}\right\rangle\left\langle B^{(n)}\right|}{2 m_{B^{(n)}}} J^{0 \dagger}(0)\right| 0\right\rangle \\
& =\sum_{n}\left\langle 0\left|J^{0}(0) e^{-H t_{x}} \frac{\left|B^{(n)}\right\rangle\left\langle B^{(n)}\right|}{2 m_{B^{(n)}}} J^{0 \dagger}(0)\right| 0\right\rangle \\
& =\sum_{n} \frac{\left|\left\langle 0\left|J^{0}(0)\right| B^{(n)}\right\rangle\right|^{2}}{2 m_{B^{(n)}}} e^{-E^{(n)} t_{x}} \\
& =\left|Z_{0}\right|^{2} e^{-E^{(0)} t_{x}}+\left|Z_{1}\right|^{2} e^{-E^{(1)} t_{x}}+\ldots
\end{aligned}
$$

where $e^{-H t_{x}}$ is the Euclidean translation operator and

$$
H\left|B^{(n)}\right\rangle=E^{(n)}\left|B^{(n)}\right\rangle
$$


The long distance behavior of $C_{2}\left(t_{x}\right)$ is dominated by the exponential associated with the lightest state $\left|B^{(0)}\right\rangle$ (with energy $E^{(0)}=m_{B}$ )

$$
C_{2}\left(t_{x}\right) \underset{t_{x} \rightarrow \infty}{\simeq}\left|Z_{0}\right|^{2} e^{-m_{B} t_{x}}
$$

Therefore by definition $n_{\mathbf{L}}^{\mathrm{T}_{1}}$

$$
Z_{0}=\frac{\left\langle 0\left|J^{0}(0)\right| B^{(0)}\right\rangle}{\sqrt{2 m_{B}}}=\frac{1}{\sqrt{2}} f_{B} \sqrt{m_{B}}
$$

Eq.(育) is not defined (yet) in the limit $t_{x} \rightarrow \infty$ because this limit is clearly large distance and therefore non-perturbative.

If we had a non perturbative definition of eq. (保), we could compute $C_{2}\left(t_{x}\right)$ and extract both $m_{B}$ and $f_{B}$ by fitting the result with eq. $\left(\begin{array}{l}1 \\ 1\end{array}\right)$.

An example of two-point correlation function computed on a lattice (using program C2.C in the Appendix) is shown in fig. II!r.

\subsection{3 -point correlation functions}

$V_{c b}$ is a very important parameter of the Standard Modele $\left.\right|_{-1} ^{2_{1}}$ It must be determined by comparing QCD contributions with experiment. By definition

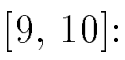

$$
\left|V_{c b}\right|^{2}=\frac{f_{B}^{2} m_{B}}{\left\langle B\left|\bar{h} \gamma^{\mu} L q \bar{h} \gamma^{\mu} L q\right| \bar{B}\right\rangle} \text { [perturbative factor][experiment] }
$$

From $C_{2}\left(t_{x}\right)$ we have the coefficients in the numerator but we also need a QCD prediction for the matrix element in the denominator. To reach this goal we define a three-point correlation function

$$
\begin{aligned}
C_{3}\left(t_{x}, t_{y}\right) & =\int \mathrm{d}^{3} \mathbf{x} \int \mathrm{d}^{3} \mathbf{y}\left\langle 0\left|J^{0}(x) \mathcal{O} J^{0}(-y)\right| 0\right\rangle \\
& =\int\left[\mathrm{d} A_{\mu}\right]\left[\mathrm{d} q_{i}\right]\left[\mathrm{d} \bar{q}_{i}\right]\left(\int \mathrm{d}^{3} \mathbf{x} \int \mathrm{d}^{3} \mathbf{y} J^{0}(x) \mathcal{O} J^{0}(-y)\right) e^{-\mathcal{S}_{\mathrm{E}}^{Q C D}}(10)
\end{aligned}
$$

\footnotetext{
${ }^{1}\left\langle 0\left|J^{\mu}\right| B\right\rangle=f_{B} p^{\mu}$

${ }^{2} V_{c b}$ is one of the elements of the Cabibbo-Kobayashi-Maskawa matrix $V$ and it is known with very poor precision. A non unitary CKM matrix would be a clear signal of physics beyond the Standard Model.
} 


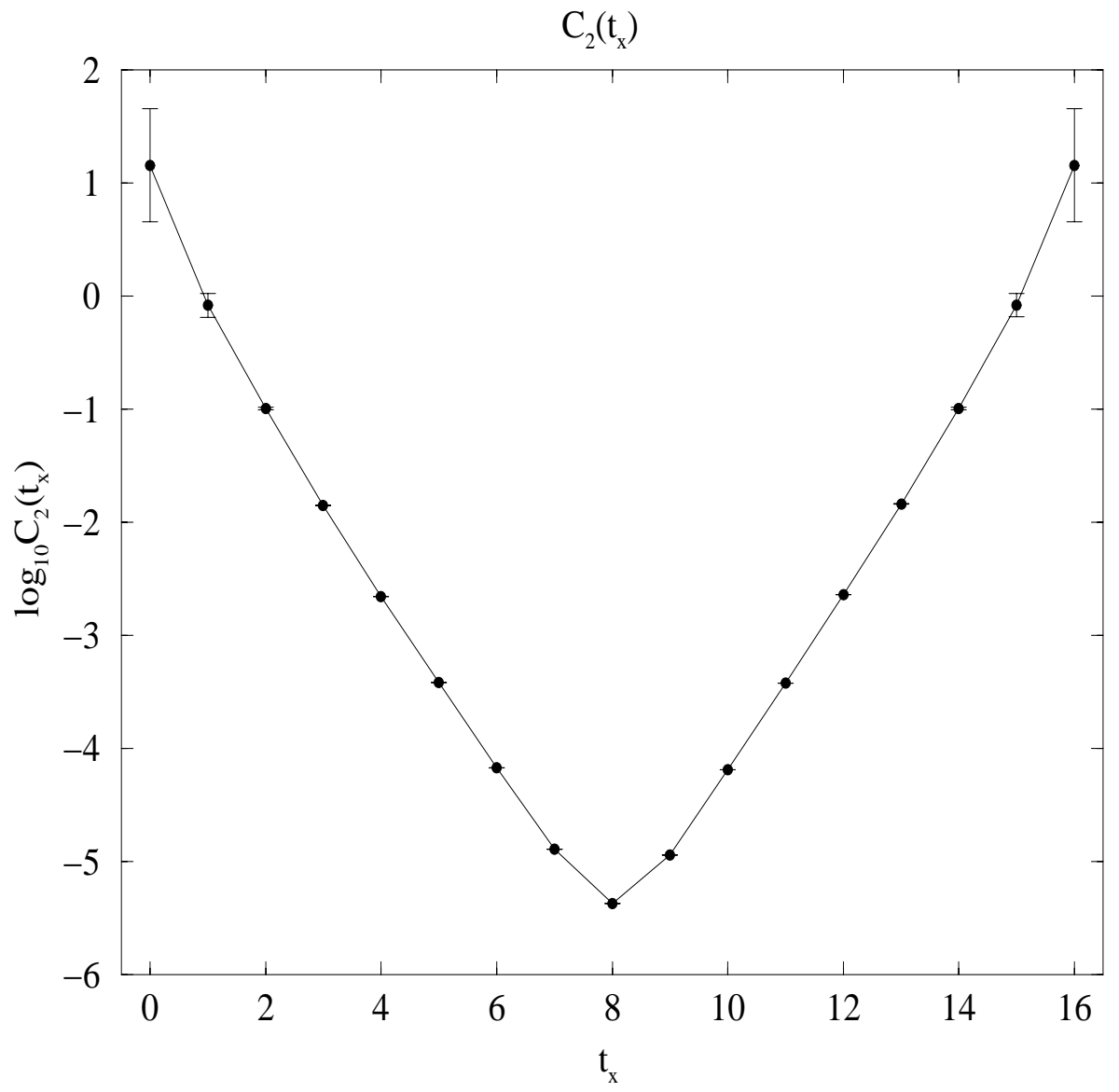

Figure 1: Example of a numerical two-point correlation function for a $B$ meson for some (arbitrary) input values of the light and heavy quark masses. The plot is symetric arout $t_{x}=8$ because of periodic boundary conditions. The curve (for $t_{x}<8$ ) can be fitted with $C_{2}(t)=\frac{1}{2} f_{B}^{2} m_{B} e^{-m_{B} t}$ to determine $m_{B}$ and $f_{B}$. 
where

$$
\mathcal{O}=\bar{h}(0) \gamma^{\mu} L q(0) \bar{h}(0) \gamma^{\mu} L q(0)
$$

(LCdefined in the appendix $\Gamma$ is the left-handed projector) We play the same trick as before (inserting two complete sets of states) and we obtain

$$
\begin{aligned}
C_{3}\left(t_{x}, t_{y}\right) & =\sum_{n, m} \int \mathrm{d}^{3} \mathbf{x} \int \mathrm{d}^{3} \mathbf{y}\left\langle 0\left|J^{0}(x) \frac{\left|B^{(n)}\right\rangle\left\langle B^{(n)}\right|}{2 m_{B^{(n)}}} \mathcal{O} \frac{\left|\bar{B}^{(m)}\right\rangle\left\langle\bar{B}^{(m)}\right|}{2 m_{B^{(m)}}} J^{0}(-y)\right| 0\right\rangle \\
& =\sum_{n, m} Z^{(n)} Z^{(m)} \frac{\left\langle B^{(n)}|\mathcal{O}| \bar{B}^{(m)}\right\rangle}{\sqrt{2 m_{B^{(n)}} 2 m_{B^{(m)}}}} e^{-E^{(n)} t_{x}} e^{-E^{(m)} t_{y}}
\end{aligned}
$$

which for $t_{x} \rightarrow \infty$ is dominated by

$$
C_{3}\left(t_{x}, t_{y}\right) \underset{t_{x}, t_{y} \rightarrow \infty}{\simeq}\left|Z_{0}\right|^{2} e^{-m_{B}\left(t_{x}+t_{y}\right)} \frac{\langle B|\mathcal{O}| \bar{B}\rangle}{2 m_{B}}
$$

Again...

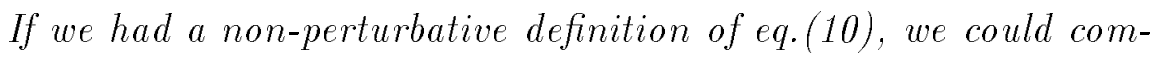
pute $C_{3}\left(t_{x}, t_{y}\right)$ and extract matrix elements (such as $\langle B|\mathcal{O}| \bar{B}\rangle$ ) by

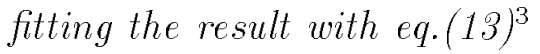

We will see in the next sections how the lattice provides both a definition

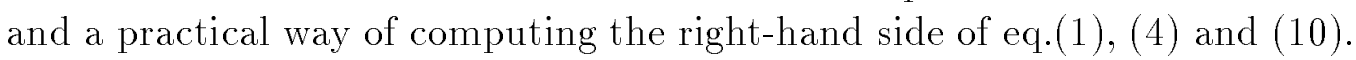

\footnotetext{
${ }^{3}$ This is what one actually does to determine $V_{c b}$. At present the main error on $V_{c b}$ is due to theoretical uncertanties.
} 


\section{Monte Carlo Integration}

Although this may seem a paradox, all exact science is dominated by the idea of approximation

(Bertrand Russell)

In this section it will be shown how to compute numerically multidimensional integrals using a statistical method (the Monte Carlo integration).

\subsection{Riemann integrable functions}

Let's consider the simplest of the integrals

$$
\int_{\alpha}^{\beta} f(x) \mathrm{d} x
$$

Riemann gave us a definition of this integral:

To check if the function $f(x)$ in the domain $\mathcal{D}=[\alpha, \beta]$ is Riemann integrable we divide the domain in $N$ small intervals

$$
\left[x_{0}=\alpha, x_{1}\right],\left[x_{1}, x_{2}\right], \ldots,\left[x_{N-2}, x_{N-1}\right],\left[x_{N-1}, x_{N}=\beta\right]
$$

and compute

$$
\varepsilon(N)=a \sum_{i=0}^{N-1} \max _{x \in\left[x_{i}, x_{i+1}\right]} f(x)-a \sum_{i=0}^{N-1} \min _{x \in\left[x_{i}, x_{i+1}\right]} f(x)
$$

where $a \stackrel{\text { def }}{=} \frac{\beta-\alpha}{N}$

$f(t)$ is Riemann integrable if $\varepsilon(N)$ goes to zero for $N \rightarrow \infty$, i.e. $a \rightarrow 0$. 


\section{Classical Integration Methods}

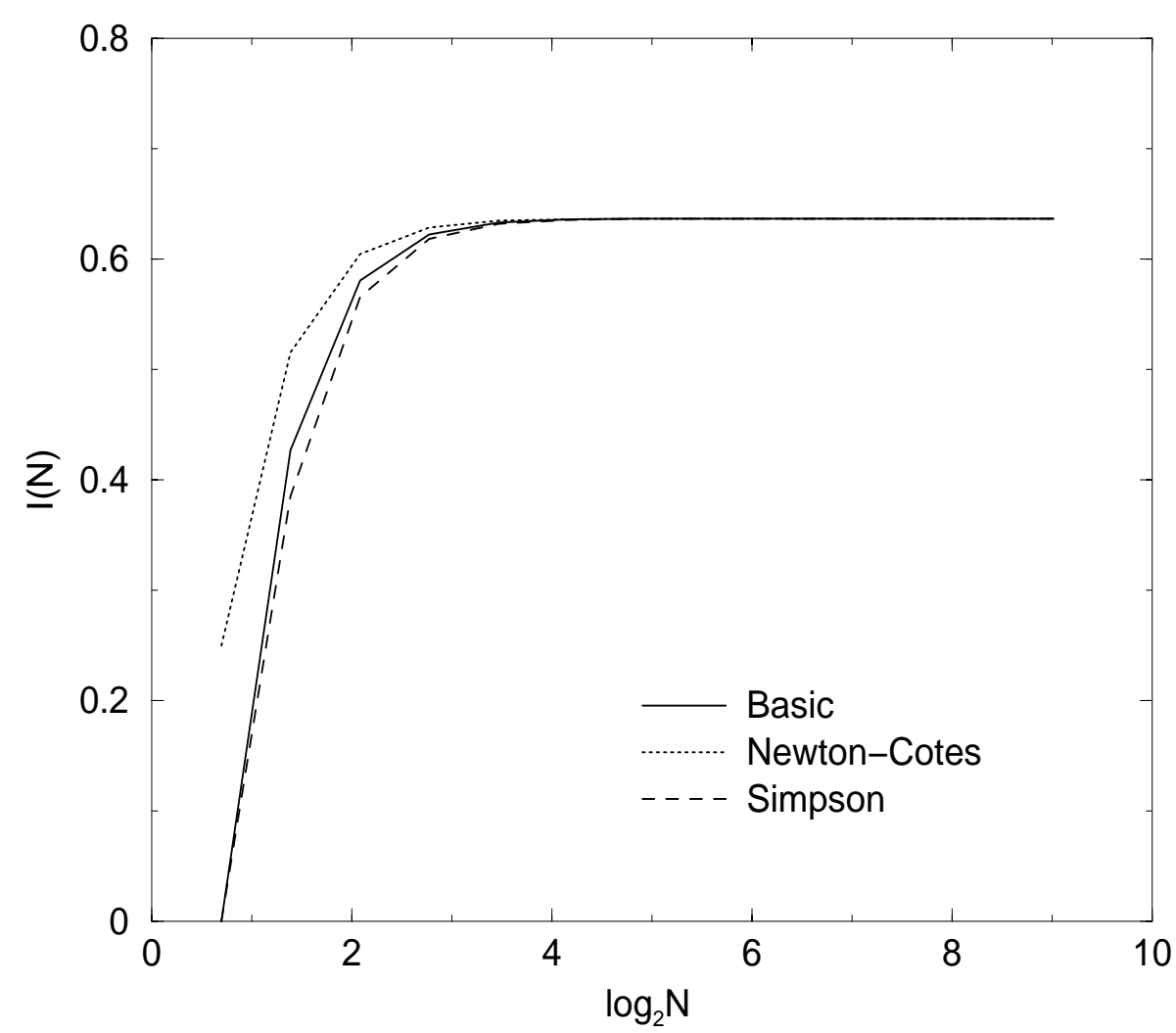

Figure 2: Convergent behavior of different numerical discretizations for the integral of eq.(1 (14 14. 
This definition of integration is also very practical because it gives a method of performing numerical integrations:

$$
\int_{\alpha}^{\beta} f(x) \mathrm{d} x=\sum_{i=0}^{N-1} a \cdot f\left(x_{i}\right)+O\left(f^{\prime} / N^{2}\right)
$$

There are other possible ways of approximating a continuum integral with a discrete sum $\mathbf{I}_{-1}^{\pi_{1}}$ and $\Gamma$ if they converge $\Gamma$ they all converge to the same number.

The difference between the different integration methods resides in the behavior of the numerical integral as function of $N$ : some methods converge faster than others. For practical purposes this is a crucial issue.

program $1 . c$ is a $\mathrm{C}$ program that computes the integral

$$
I=\int_{0}^{1} \sin (\pi x) \mathrm{d} x \simeq 0.63662
$$

using three different simple methods. The convergent behavior of the different methods is shown in fig.1. The error done when one stops at a finite $N$ is known as disctretization error.

For multidimensional integrals this method turns out to be too slow for practical applications. Therefore we need a new tool M Monte Carlo integration.

\subsection{Basic Monte Carlo}

Monte Carlo integration is another numerical method for integration and it has a statistical foundation. The algorithm is the following:

${ }^{4}$ for example using trapezoids instead of rectangles (Newton-Cotes method)

$$
\int_{\alpha}^{\beta} f(x) \mathrm{d} x=\sum_{i=0}^{N-1} a \cdot \frac{f\left(x_{i}\right)+f\left(x_{i+1}\right)}{2}+O\left(f^{\prime \prime} / N^{3}\right)
$$

or using interpolating polynomia (Simpson's method)

$$
\int_{\alpha}^{\beta} f(x) \mathrm{d} x=\sum_{i=0}^{N-2} a \cdot \frac{f\left(x_{i}\right)+4 f\left(x_{i+1}\right)+f\left(x_{i+2}\right)}{6}+O\left(f^{\prime \prime \prime \prime} / N^{5}\right)
$$

See the Numerical Recipes for more details. 
- Generate a set of $N$ random points $\left\{x^{[i]}\right\}$ with uniform distribution in the integration domain $\mathcal{D}$

- For each point $x^{[i]}$ compute $f\left(x^{[i]}\right)$

- Compute the average $I(N)=\frac{\beta-\alpha}{N} \sum_{i=0}^{N-1} f\left(x^{[i]}\right)$

If $f(x)$ is Riemann integrable, the function $I(N)$ converges to the integral of $f(x)$ when $N \rightarrow \infty$.

program 2. $\mathrm{c}$ is a $\mathrm{C}$ program that computes numerically Tusing Monte Carlo integration $\Gamma$ the same integral of eq. (1) $(\overline{1})$. Its convergent behavior is shown in fig. 2 and compared with the standard methods showed in section 2.1.

This algorithm can easily be extended to arbitrary dimensions. For example program3.c computes the following integral

$$
I=\int_{0}^{1} d x_{0} \int_{0}^{1} d x_{1} \int_{0}^{1} d x_{2}\left(3 x_{0}^{2} x_{1}+2 x_{2}^{3}\right)=1
$$

and its convergent behavior is shown in fig.3. Monte Carlo integration is not very efficient for $1 \mathrm{D}$ integrals but it becomes more and more efficient (when compared with conventional integration methods) for higher dimensional integrals.

From now on we will use the upper index $\mathrm{x}^{[i]}$ to label the Monte Carlo points and a lower index $x_{j}$ to label a particular coordinate of a given point. For example $x_{2}^{[3]}$ is the second coordinate of the 3 rd point.

\subsection{Metropolis Monte Carlo}

In the next section we will deal with $K$-dimensional integrals of the form

$$
\begin{gathered}
I_{k}=\int_{0}^{1} d x_{0} \int_{0}^{1} d x_{1} \ldots \int_{0}^{1} d x_{K-1} f(\mathbf{x}) P(\mathbf{x}) \\
\text { where } \mathbf{x}=\left(x_{0}, x_{1}, \ldots, x_{K-1}\right)
\end{gathered}
$$

and we have to compute them for different functions $f(\mathrm{x})$ but the same $P(\mathbf{x}){ }_{1}^{5}$. The clever trick we play is to modify our algorithm in the following way:

\footnotetext{
${ }^{5}$ think for example to the case of many Euclidean green functions $G_{k}$ defined as

$$
G_{k}=\int[\mathrm{d} \mathbf{x}] f_{k}(\mathbf{x}) P(\mathbf{x})
$$
}




\section{Monte Carlo Integration Method}

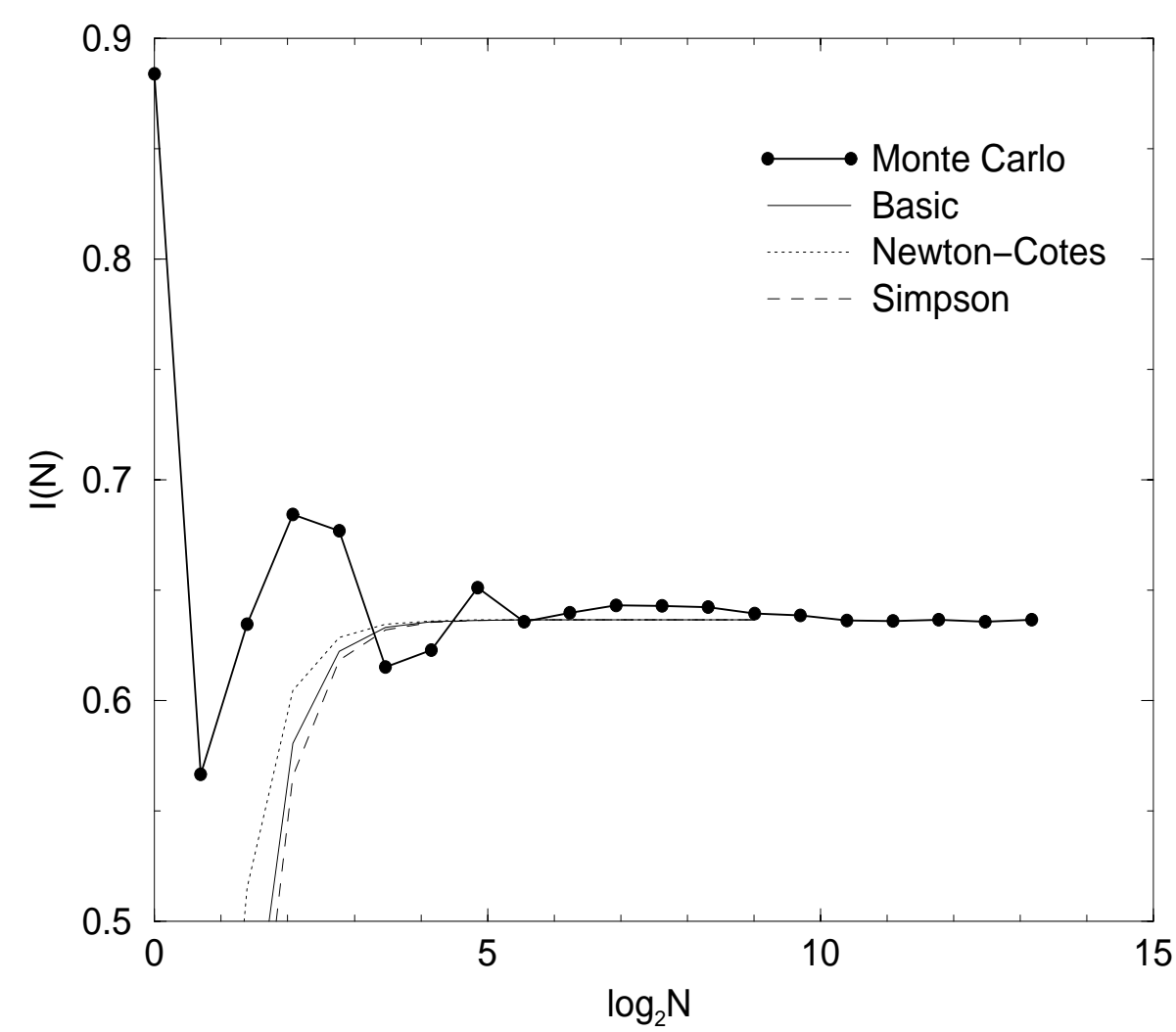

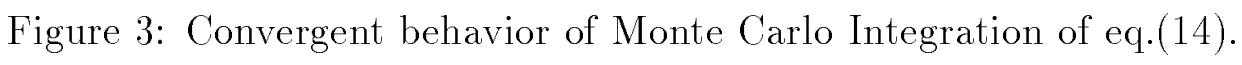




\section{Multidimensional Monte Carlo Integration}

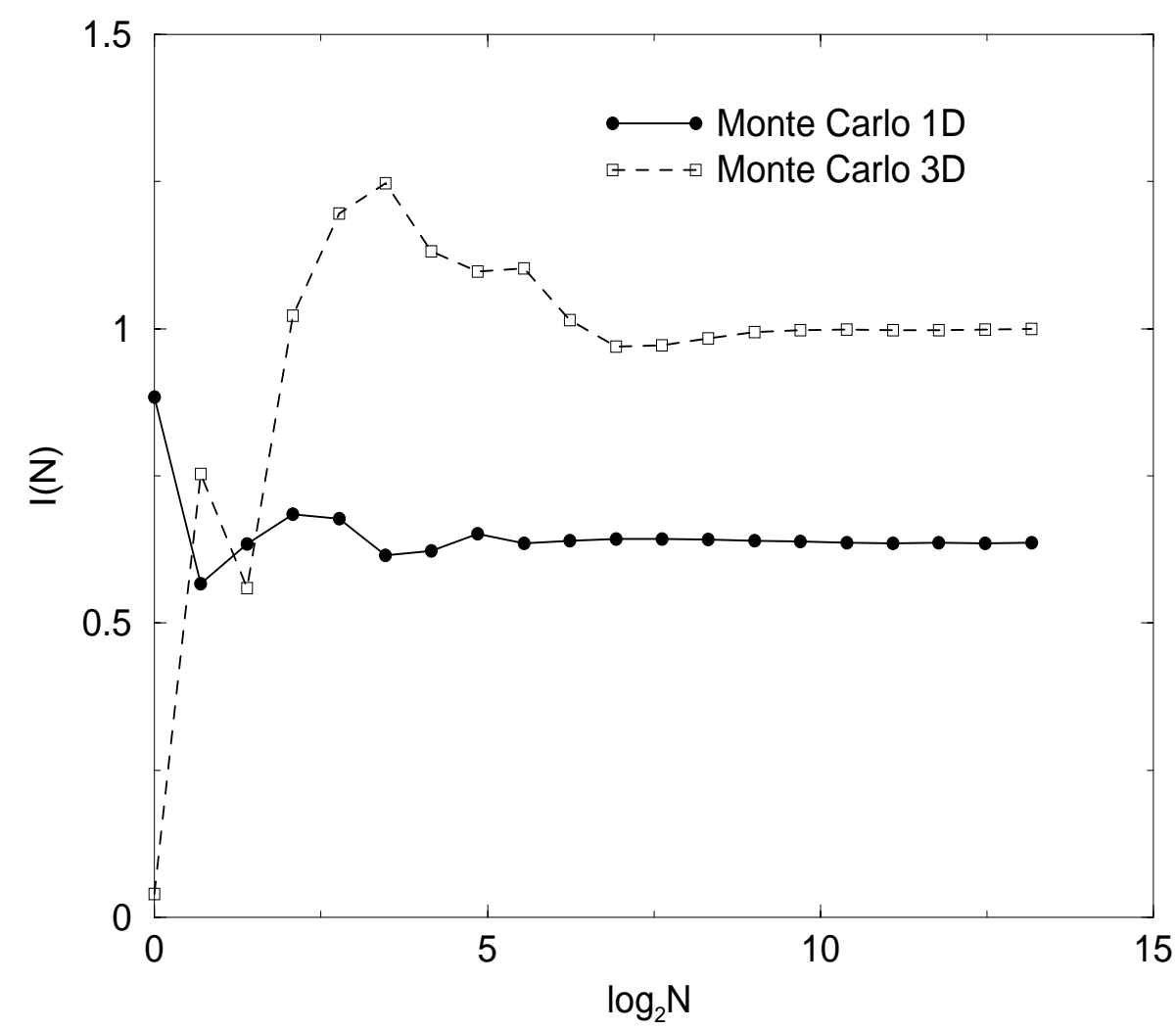

Figure 4: Convergent behavior Monte Carlo Integration of eq.(120in) 
- Generate a set of $N$ random points $\left\{\mathrm{x}^{[i]}\right\}$ in the integration domain $\mathcal{D}$ using $P\left(\mathrm{x}^{[i]}\right)$ for the probability distribution of the point $\mathrm{x}^{[i]}=$ $\left(x_{0}^{[i]}, x_{1}^{[i]}, \ldots, x_{K-1}^{[i]}\right)$

- For each point $\mathrm{x}^{[i]}$ compute $f\left(\mathrm{x}^{[i]}\right)$

- Compute the average

$$
I(N)=\frac{\operatorname{Vol}(\mathcal{D})}{N} \sum_{i=0}^{N-1} f\left(\mathrm{x}^{[i]}\right)
$$

where $\operatorname{Vol}(\mathcal{D})$ is the volume of the integration domain.

If $f(\mathrm{x}) P(\mathrm{x})$ is Riemann integrable then the function $I(N)$ converges to the integral eq. (商) when $N \rightarrow \infty$. The factor $P(\mathrm{x})$ in the integrand has been absorbed into the probability distribution of generating the random points.

We are now left with the problem of generating random points $\mathrm{x}^{[i]}$ with a given distribution $P\left(\mathrm{x}^{[i]}\right)$. There are many algorithms that do this job. The simplest one is the Metropolis algorithm [i]

1. Start with $i=0$ and a point $\mathrm{x}^{[i]}$ chosen at random in the integration domain.

2. Generate another random point $\mathbf{y}$ in the integration domain and a random number $\alpha$ in the interval $[0,1)$.

3. If $P(\mathbf{y}) / P\left(\mathrm{x}^{[i]}\right)>\alpha$ then $\mathrm{x}^{[i+1]}=\mathbf{y}$ else $\mathrm{x}^{[i+1]}=\mathrm{x}^{[i]}$.

4. Increase $i$ by 1 and repeat steps 2 I3R.

The succession of points $\left\{\mathrm{x}^{[i]}\right\}$ generated by this algorithm has the required probability $P\left(\mathrm{x}^{[i]}\right)$. This kind of succession is also known as Markov chain.

Each point of the Markov chain is called a configuration.

where $P(\mathbf{x}) \propto e^{-S_{E}(\mathbf{x})}$ and $S_{E}(\mathbf{x})$ is the Euclidean action of a system in the configuration $\mathbf{x}$. 


\section{Metropolis MC Algorithm}

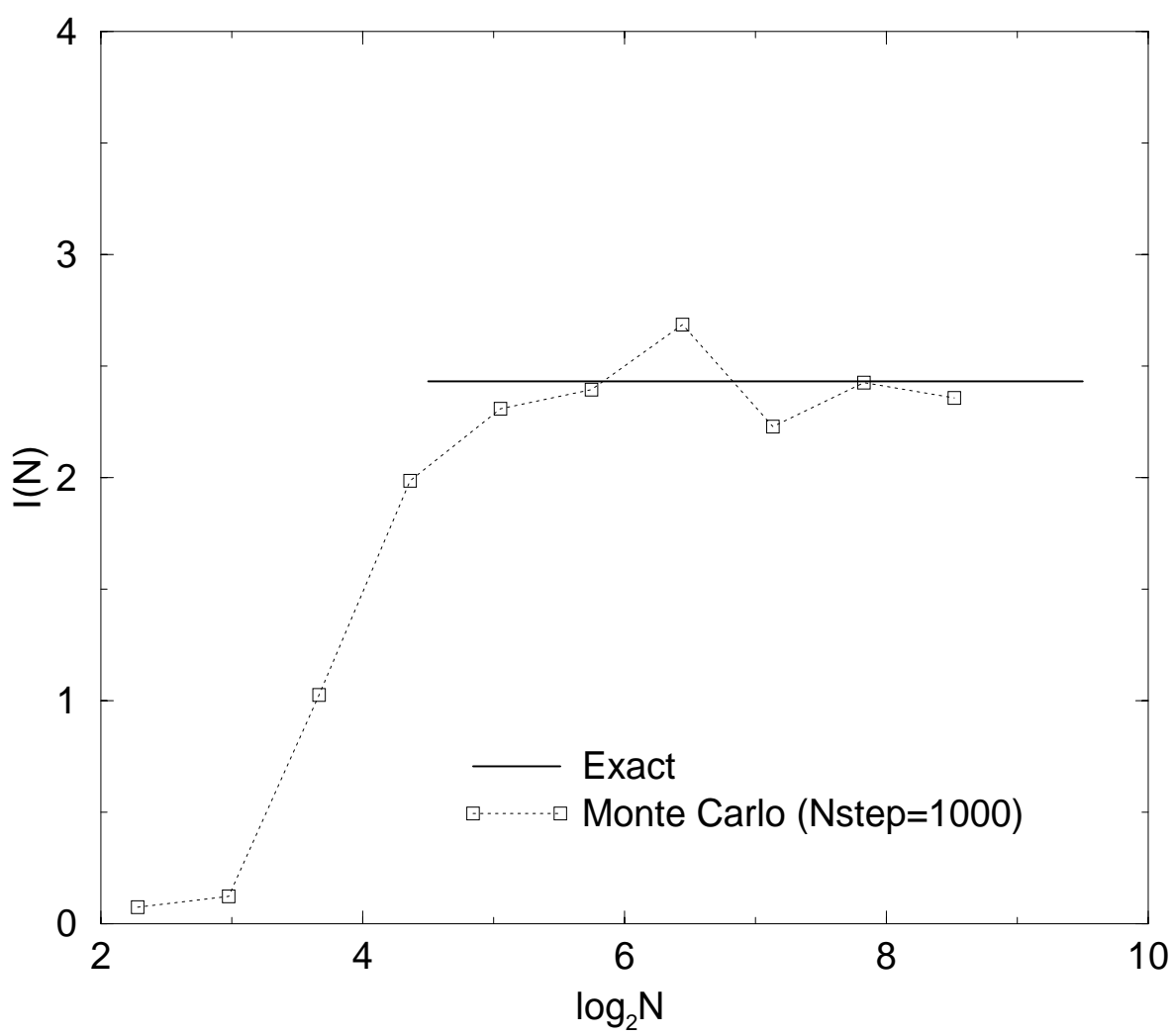

Figure 5: Convergent behavior of the Metropolis algorithm. 
The Metropolis Monte Carlo only works with a real probability function $\Gamma$ $P(x)$ Tbut the functions $f(x)$ Tand the integration variables $\mathrm{x}$ Гcan be complex $\Gamma$ vectors or tensors.

program4.c computes the following 3D integra $\left.\right|_{-1} ^{\sigma_{1}^{1}}$

$$
\begin{gathered}
I=\int_{0}^{1} d x_{0} \int_{0}^{1} d x_{1} \int_{0}^{1} d x_{2} f(\mathbf{x}) P(\mathbf{x}) \\
\text { where } P(\mathbf{x})=e^{-\left(x_{0}^{2}+x_{1}^{2}+x_{2}^{2}\right)} \\
\text { and } f(\mathbf{x})=128 x_{0}^{3} x_{1}^{2} x_{2}
\end{gathered}
$$

Its convergent behavior is shown in fig. 4 .

\subsection{Bootstrap errors}

Monte Carlo integration does not just provide us with a way to compute multidimensional integrals. There are two techniques $\Gamma$ known and Jackknife and Bootstrap [1:2i] That permit us to evaluate the statistical error we commit when we truncate the Markov chain and we compute the numerical integral on a finite number of configurations $\Gamma N$. We consider here the Bootstrap method.

Let's assume we have a finite number $\Gamma N$ Tof configurations that constitute the Markov chain $\left\{\mathrm{x}^{[i]}\right\}$ and the integral $I$ which is computed numerically as

$$
I \simeq I(N) \stackrel{\text { def }}{=} \frac{\operatorname{Vol}(\mathcal{D})}{N} \sum_{i=0}^{N-1} f\left(\mathrm{x}^{[i]}\right)
$$

Since in general the $f\left(\mathrm{x}^{[i]}\right)$ are not Gaussian distributed, standard error analysis is not applicable.

The Bootstrap algorithm consists of the following steps:

- Construct a table of $N \times M$ integer random numbers $k_{i j}$ where $i \in$ $\{0,1, \ldots, N-1\} \Gamma j \in\{0,1, \ldots, M-1\}$ and $k_{i j} \in\{0,1, \ldots, N-1\}$ for each couple $(i, j)$ ( $M$ is an input parameter that we choose to be equal to $100)$.

\footnotetext{
${ }^{6}$ it can easily be computed analytically by factorization and one finds $I=2.43131$
} 
- For each $j$ compute

$$
\bar{I}_{j} \stackrel{\text { def }}{=} \frac{\operatorname{Vol}(\mathcal{D})}{N} \sum_{i=0}^{N-1} f\left(\mathrm{x}^{\left[k_{i j}\right]}\right)
$$

- Reorder the set $\left\{\bar{I}_{j}\right\}$ so that $\Gamma$ for each $j \Gamma \bar{I}_{j}<\bar{I}_{j+1}$

The result for the integral I lies between $\bar{I}_{33}$ and $\bar{I}_{66}$ at $65 \%$ confidence level.

The idea behind the Bootstrap algorithm is that $\Gamma$ for each $j \Gamma$ and for $N \gg 1 \Gamma$ the $j$-th Markov chain $\left\{\mathbf{x}^{\left[k_{i j}\right]}\right\}$ (chain in $i$ ) has the same probability distribution of the original chain $\left\{\mathrm{x}^{[i]}\right\}$. Therefore the different $\bar{I}_{j}$ (the numerical integral computed using the derived chain $j$ ) have the same probability distribution as that of the derived chain $j$ associated to $\bar{I}_{j}$.

program5.c implements the Bootstrap algorithm as complement of the Metropolis algorithm. Fig.5 shows the same data of fig. 4 including the Bootstrap error. The kind of error one does when truncates the Markov chain is known as statistical error. 


\section{Metropolis MC with Boostrap Errors}

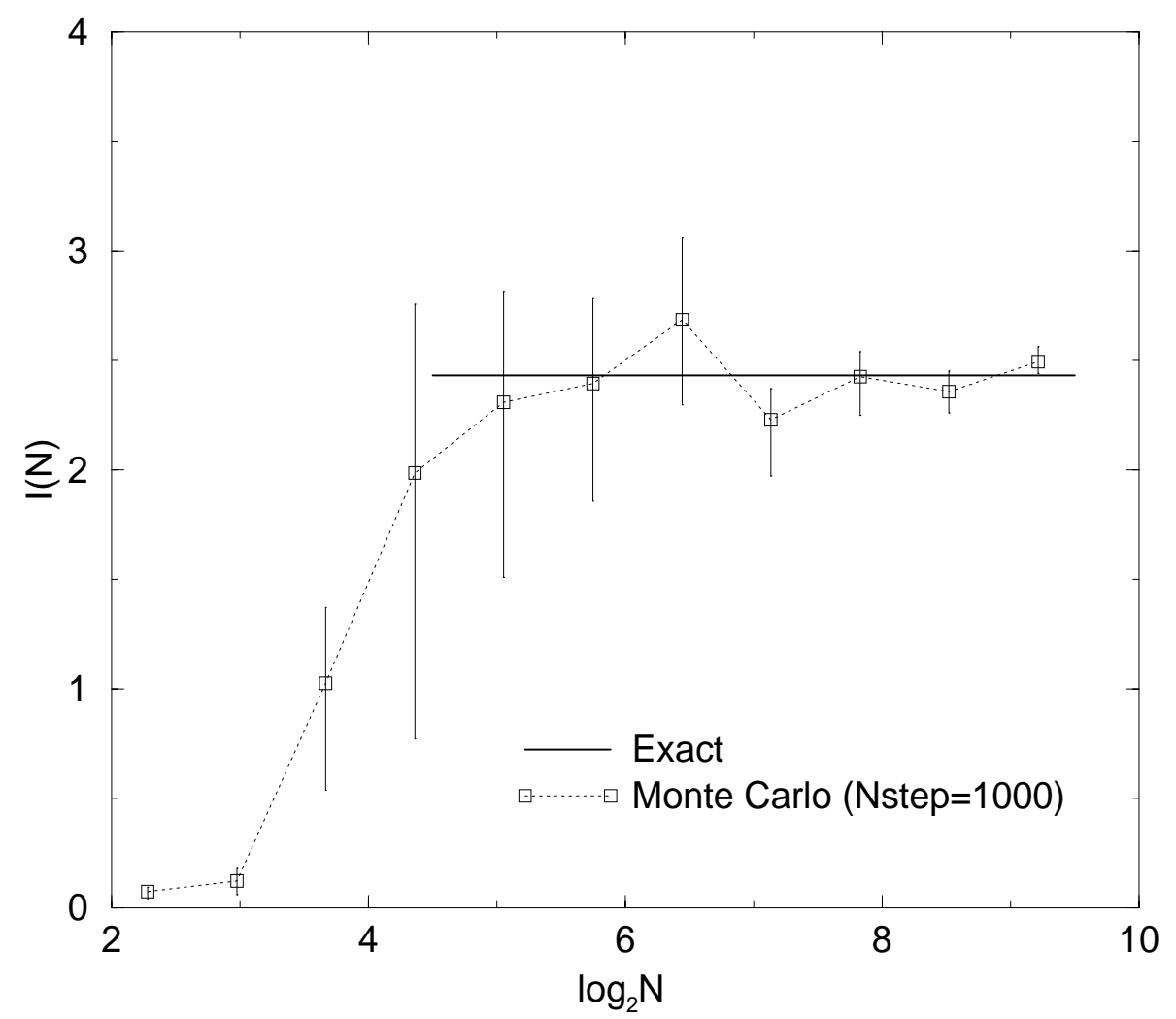

Figure 6: Convergent behavior of the Metropolis and Bootstrap algorithm. 


\section{Definition of Path Integrals}

Calculus required continuity, and continuity was supposed to require the infinitely little; but nobody could discover what the infinitely little might be

(Bertrand Russell)

The concepts of Regularization and Renormalization play a fundamental role in the definition of the Path Integral (and in physics in general)

There is a physical reason for it:

- One never measures the value of a field (associated to a particle) in every point in space-time but one measures its integral over the test function of the physical detectors which have a finite extension. Therefore there are mathematical reasons to require that the fields are defined in the space of distributions.

- One wants to model the unknown short distance physics by introducing a Lagrangian density which contains only local (contact) interactions.

If one tries to combine the previous statements in a Quantum Field Theory with them. The mathematical origin of these divergences is the presence of undefined formal products of distributions in the Lagrangian from which the path integral is computed. Regularization and Renormalization are in fact $\Gamma$ in mathematical terms Tthe solution to the problem of defining these products of distributionsit.

We analyze here $\Gamma$ as an explanatory example $\mathrm{T}$ the problem of defining the product of $\delta$ functions by regularizing them by a sequence of smooth functions that become more localized at zero. We then show how an arbitrary quantum field can be expanded and regularized using delta functions and

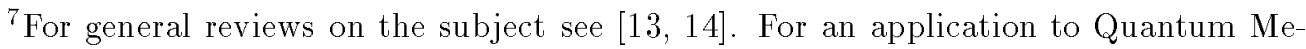
chanics see [1] 1 in
} 
how the presence of a finite spatial cut-off in the regularized distributions is equivalent to a finite cut-off in the momentum expansion of the field itself.

This is not intended to be an introduction to Renormalization Tbut it is presented as an alternative view of its meaning having in mind the lattice as typical regulator.

\subsection{Toy example: Regularizing distributions}

The $\delta$ function is a distribution which is defined as

$$
\int \delta(x-\bar{x}) F(x) \mathrm{d} x=F(\bar{x})
$$

for any smooth test function $F(x)$. The same $\delta$ function can be thought of as the limit of an ordinary function $\delta(a, x)$

$$
\delta(x)=\lim _{a \rightarrow 0} \delta(a, x)
$$

where $\delta(a, x)$ must be enough regular Tlocalized in $x$ within a precision $a$ and its integral normalized to one. This procedure is called regularization. Some possible regularization schemes are $\mathrm{L}_{-1}^{\nabla_{1}}$

$$
\begin{aligned}
\delta(a, x) & =\frac{1}{a}[\theta(x+a / 2)-\theta(x-a / 2)] \\
\delta(a, x) & =\left(\pi a^{2}\right)^{-\frac{1}{2}} \exp \left(-x^{2} / a^{2}\right) \\
\delta(a, x) & =\frac{\sin (\pi x / a)}{\pi x}
\end{aligned}
$$

They are sketched in figure sense that they give the same result for the following limit

$$
\lim _{a \rightarrow 0} \int \delta(a, x-\bar{x}) F(x) \mathrm{d} x=F(\bar{x})
$$

but they do not give a well defined limit in expressions of the form

$$
\lim _{a \rightarrow 0} \int[\delta(a, x-\bar{x})]^{n+1} F(x) \mathrm{d} x=?
$$

\footnotetext{
${ }^{8}$ The $\theta(x)$ function is defined to be 0 for $x<0$ and 1 for $x>0$. It is discontinuous in $x=0$.
} 

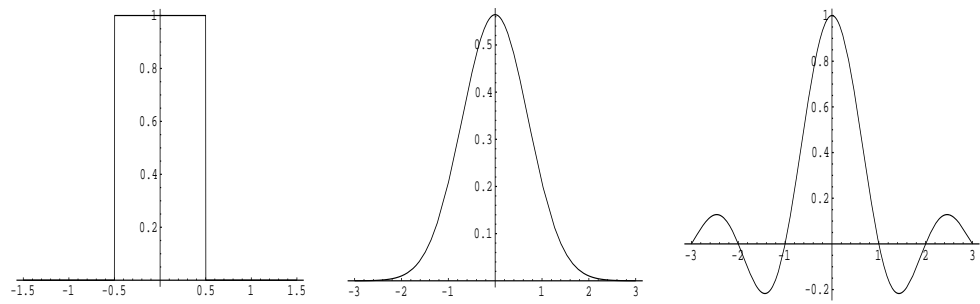

Figure 7: Examples of possible regularizations for a delta function. The $x$ axis is in units of $a$ The $y$ axis is in units of $a^{-1}$.

In fact eq. $\left(\overline{\bar{\beta}}_{\underline{3}} \overline{4}_{1}^{4}\right)$ is divergent as $a^{-n}$. Suppose one wants to give a well defined meaning to the limit in eq. (3) by removing somehow the divergence that occurs. One way of doing it is by making $F(x)$ dependent on $a$ Taccording with the prescription for $\delta(a, x) \Gamma$

$$
F(x) \rightarrow F_{R}(a, x)=Z_{R}^{-n}(a) F(x)
$$

where $Z_{R}^{-1}(a)=a+O\left(a^{2}\right)$. In other words the divergence of the integral is absorbed in the normalization of the function $F(x)$. This procedure is called renormalization and it depends on which regularization has been chosen. From now on the scheme of eq. $\left(\begin{array}{c}\overline{3} \\ 3 \\ 0\end{array}\right)$ will be considered in particular. After renormalization

$$
\int[\delta(a, x-\bar{x})]^{n+1} Z_{R}^{-n}(a) F(x) \mathrm{d} x=\text { const. }+O(a)
$$

and its limit for $a \rightarrow 0$ becomes well defined. Therefore $\mathrm{up}$ to order $a$ terms one can redefine the integral of eq. $\left(\overline{\overline{3}}_{\underline{3}} \bar{q}_{1}^{\prime}\right)$ in the following way

$$
\int[\delta(x-\bar{x})]^{n+1} F(x) \mathrm{d} x \stackrel{\text { def }}{=} \lim _{a \rightarrow 0} \int[\delta(a, x-\bar{x})]^{n+1} Z_{R}^{-n}(a) F(x) \mathrm{d} x
$$

The situation can be even more complicated if $F(x)$ itself is defined in terms of delta functions. For example one can consider the case when $F(x)=$ $\exp \left[g \delta^{2}(x)\right]$. In this case it is not sufficient to regularize $\delta$ and renormalize $F$ to get rid of the divergence 5 one is forced to renormalize $g$ as well.

It is a general statement that $\Gamma$ if the function $F(x, g)$ depends on some constant $g$ Tone has to renormalize the constant

$$
g \rightarrow g_{R}(a)
$$


by imposing a constraint

$$
\int[\delta(a, x-\bar{x})]^{n+1} Z_{R}^{-n}(a) F\left(x, g_{R}(a)\right) \mathrm{d} x=\text { const. }
$$

Eq. ( $(3 \overline{9})$ fixes the behavior of $g_{R}(a)$ as function of $a$. Its solution $\Gamma g_{R}(a) \Gamma$ can have a non-trivial behavior in $a$. Eq. ( $(39)$ is a particular case of what is generally known as the Renormalization Group Equation (RGE) [A- The behavior of $g_{R}(a)$ versus $a$ is called running. Another common way of writing the renormalization group equation is

$$
\frac{\mathrm{d}}{\mathrm{d} \log a} \int[\delta(a, x-\bar{x})]^{n+1} Z_{R}^{-n}(a) F\left(x, g_{R}(a)\right) \mathrm{d} x=0
$$

or explicitly

$$
\left(a \frac{\partial}{\partial a}-\beta\left(g_{R}\right) \frac{\partial}{\partial g_{R}}+n \gamma\left(g_{R}\right)\right) \int[\delta(a, x-\bar{x})]^{n+1} Z_{R}^{-n} F\left(x, g_{R}\right) \mathrm{d} x=0
$$

where

$$
\begin{aligned}
& \beta\left(g_{R}\right) \stackrel{\text { def }}{=}-\left.\frac{\partial}{\partial \log a} g_{R}(a)\right|_{g_{R}} \\
& \left.\gamma\left(g_{R}\right) \stackrel{\text { def }}{=} \frac{\partial}{\partial \log a} Z_{R}(a)\right|_{g_{R}}
\end{aligned}
$$

If the original constant $g$ is dimensionless $\Gamma g_{R}(a)$ must also depend on some other scale $\Gamma$ say $\Lambda \Gamma$ to cancel the dimension of $a$. In other words $g_{R}$ must be a function of $a \Lambda$ Tan adimensional quantity. This simple example shows how the renormalization procedure may force one to introduce a second scale $\Lambda$ of which the renormalized constant is a function. This phenomenon is called dimensional transmutation.

Dimensional transmutation is also a characteristic of QCD (and gauge theories in general). In typical physical problems $\Gamma a$ is a free parameter and it can be chosen (the physics does not depend on it providing it is small enough). $\Lambda$ Ton the other side characterizes the typical scale of the physics one is describing. If one measures $g_{R}(a \Lambda)$ at some arbitrary physical scale $a=\bar{a}$ one can uniquely determine the value of $\Lambda$. Once $\Lambda$ is known one can predict the value of $g_{R}$ at any other scale $a$. 

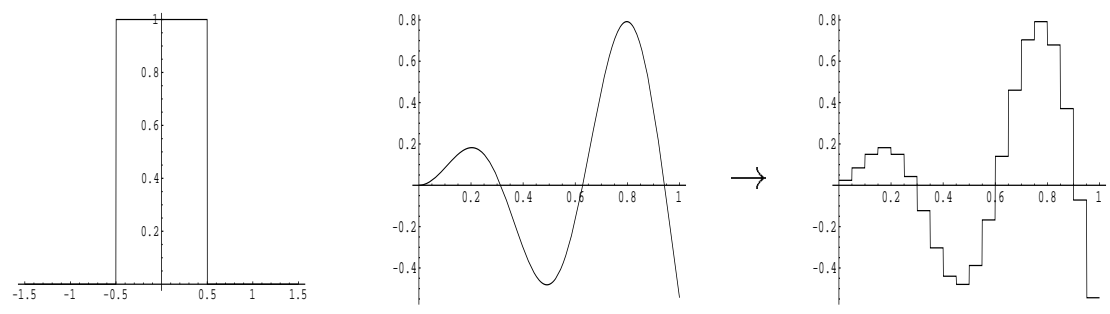

Figure 8: [left]:Example of a possible regularizations for the delta function. The $x$ axis is in units of $a$ Tthe $y$ axis is in units of $a^{-1}$. [center-right]:Example of a continuum field configuration $\phi$ and its approximation with a linear combinations of regularized delta functions.

\subsection{Defining the Path Integral}

We now go back to the most general correlation function $\Gamma$ defined in terms of the Path Integral. We rewrite it as

$$
\left\langle 0\left|T\left\{\phi\left(x_{1}\right) \ldots \phi\left(x_{n}\right)\right\}\right| 0\right\rangle \stackrel{\text { def }}{=} \int[\mathrm{d} \phi] F[\phi(x), g]
$$

where $F[\phi(x), g]$ is the integrand

$$
F[\phi(x), g] \stackrel{\text { def }}{=} \phi\left(x_{1}\right) \ldots \phi\left(x_{n}\right) e^{-\mathcal{S}_{\mathrm{E}}[\phi, g]}
$$

and $g$ is the coupling constant that appears in the action. For the moment we simply consider a one-dimensional scalar field theory $\phi(x)$ defined in the interval $[0, L]$. The specific form of the action $\Gamma S_{E}[\phi, g]$ is unimportant but we assume that the action contains an interaction term of the form

$$
g \int \phi^{n}(x) \mathrm{d} x
$$

with $n>2$. This makes $F[\phi, g]$ a non-trivial functional of $\phi(x)$.

"Lattice regularization" is the way to regularize the integral (14 by approximating the fields with sums of regularized distributions. This is equivalent to discretize the space-time on which the fields are defined. 
For $a=0.1(K=10)$ :

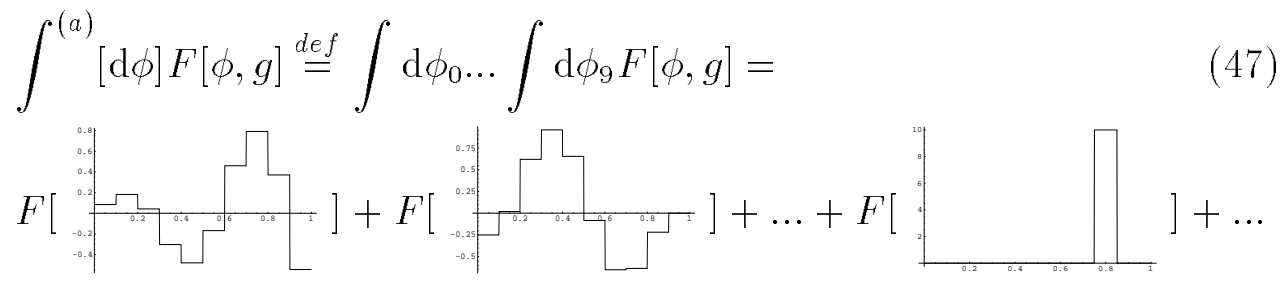

For $a=0.05(K=20)$ :

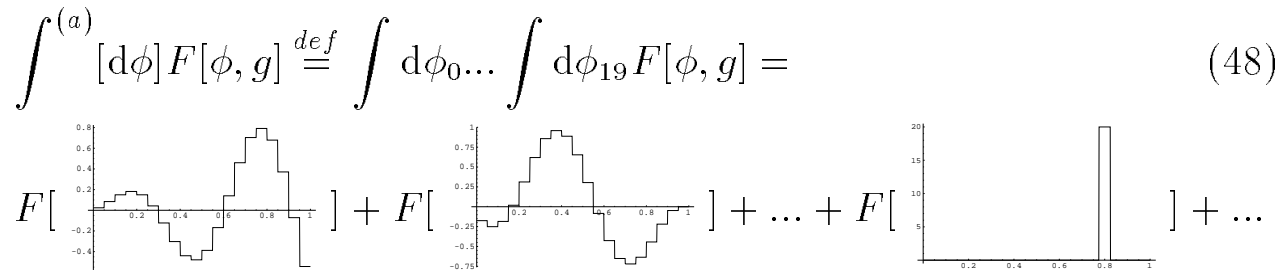

For $a=0.025(K=40)$ :

$\int^{(a)}[\mathrm{d} \phi] F[\phi, g] \stackrel{\text { def }}{=} \int \mathrm{d} \phi_{0} \ldots \int \mathrm{d} \phi_{39} F[\phi, g]=$

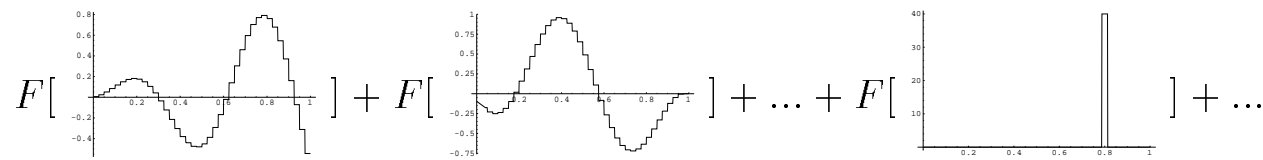

And at the continuum limit $\Gamma a \rightarrow 0(K \rightarrow \infty)$

$$
\int[\mathrm{d} \phi] F[\phi, g] \stackrel{\text { def }}{=} \lim _{a \rightarrow 0} \underbrace{\int \mathrm{d} \phi_{0} \ldots \int \mathrm{d} \phi_{K-1}}_{K \simeq L / a} F[\phi, g]=
$$

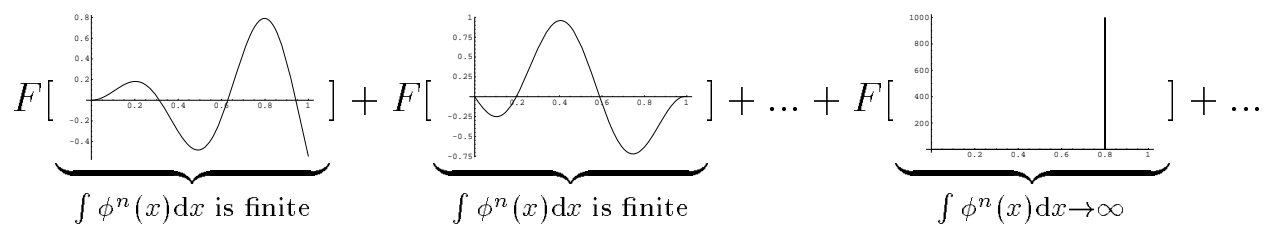

Figure 9: Example of lattice regularization of the Path Integral 
In practice one introduces a mimimum lenght scale the "lattice spacing" $a$ Tand expresses the most general path (field configuration) $\phi(x)$ in terms of regularized delta functions (as shown in fig. 条).

$$
\phi(x) \simeq \phi^{\mathrm{latt}}(a, x) \stackrel{\text { def }}{=} \sum_{k=0}^{K-1} \phi_{k} \delta(a, x-k a)
$$

where

$$
\phi_{k} \stackrel{\text { def }}{=} \frac{1}{a} \int_{k a}^{k a+a} \phi(x) \mathrm{d} x
$$

are ordinary integration variables and $\delta(a, x)$ is a regularized delta function (it is normalized to one and peaked at zero within the length scale $a \Gamma$ fig. 's

With this prescription $\Gamma$ for every finite $a=L / K$ Tone can define

$$
\int^{(a)}[\mathrm{d} \phi] F[\phi(x), g] \stackrel{\text { def }}{=} \underbrace{\int \mathrm{d} \phi_{0} \int \mathrm{d} \phi_{1} \ldots \int \mathrm{d} \phi_{K-1}}_{K \simeq L / a} F\left[\phi^{\mathrm{latt}}(a, x), g\right]+O(a)
$$

Note the upper index $(a)$ which identifies the regularized Path Integral with lattice spacing set to $a$.

Due to discretization The continuum variable $x$ has been replaced by the discrete index $k$.

For every finite $K$ Tthe right-hand side of eq. $(\overline{\bar{p}} \overline{3} \overline{3})$ can be computed using the Monte Carlo technique discussed in the preceding chapter. In this case the integration variables $\phi_{k}$ replace the $x_{k}$ of the last chapter.

The regularized Path Integral, eq. ( $\bar{B} \overline{3})$, is well defined for any finite $a$. The limit $K \rightarrow \infty$ is infested by divergences and we must give a prescription to make sense to this limit.

Divergences associated with limit $a \rightarrow 0, K \rightarrow \infty$ (at $L=K a=$ constant $)$ are called ultraviolet, while those associated with limit $K, L \rightarrow \infty$ (at $a=L / K=$ constant) are called infrared.

Fig. 19 shows in a schematic way ${ }_{-1}$ show the path integral $\Gamma$ eq. $(50)$ can can

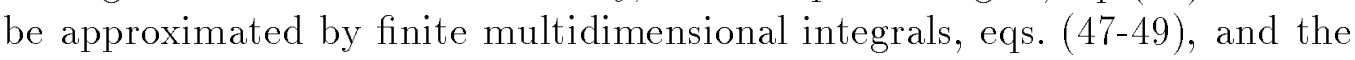


fields are defined on a lattice. For each finite $a$ Tthe "infinite sum on the path" (eqs. $(\overline{4} \overline{7}-\overline{4} \overline{4} \overline{9})$ ) is well defined since all the divergences that may appear can be absorbed in the normalization of the integration measure and $\Gamma$ for each path $\phi$ The functional $F[\phi, g]$ is finite. In the limit $a \rightarrow 0$ the regularized delta function $\delta(x, a)$ become more and more peaked and approaches a Dirac $\delta(x)$ function (eq. $(50,0)$ ). Since the integrand $F[\phi(x), g]$ is non-linear in the field and the product of delta functions is not defined $\Gamma F[\phi, g]$ diverges on those configurations $\phi(x) \simeq \delta(x)$. Therefore the Path Integral diverges.

These divergences are not physical since $\Gamma$ in any practical experiment $\Gamma$ one only has a finite resolution $\Gamma \bar{a}$ Tand one cannot discriminate a $\delta(x)$ form $\delta(x, a)$ if $a<\bar{a}$. This is why one is allowed to substract these divergences or ignore them by considering the regularized theory an "effective theory".

The way out is the following:

To have a well defined limit $a \rightarrow 0, K \rightarrow \infty$ (at $L=K a=$ constant $)$ one must impose that the result of the regularized path integral is independent from a. The only way to do it is to make the field normalization and the coupling constant (the g of eq. $(\dot{y} \bar{q})$ ) dependent on a

$$
g \rightarrow g_{R}(a, \Lambda)
$$

(the constant $\Lambda$ must be introduced because in general a and $g$ do not have the same dimensions). This makes the physics independent by the lattice scale a.

One does it by choosing a particular correlation function (identified by the functional integrand $F[\phi, g]$ and imposing the contraint

$$
\frac{\mathrm{d}}{\mathrm{d} \log a}[\underbrace{\int \mathrm{d} \phi_{0} \int \mathrm{d} \phi_{1} \ldots \int \mathrm{d} \phi_{K-1}}_{K \simeq L / a} F\left[\phi(x), g_{R}(a, \Lambda)\right]] \simeq 0
$$

This determines the behavior (the running) of $g_{R}(a)$. Eq. (5) is nothing else than a way to write the Renormalization Group Equation for a lattice regularized theory. The appearence of $\Lambda$ is called dimensional trasmutation.

The effects of very short distance physics (below the length scale $a$ ) only appear in the regularized correlation functions through corrections which are proportional to $a$ Tor through the renormalization of the coupling (and $\Gamma$ in general $\Gamma$ of the masses) [i] $\overline{1}]$. 
It should be remarked that this procedure of defining the limit $a \rightarrow 0$ cannot be carried out for an arbitrary theory since there may be more sources of divergences than coupling constants. If this limit can be defined the theory is said to be renormalizable.

Usually one distinguishes between the "bare" parameters that appear in the regularized Lagrangian (for a finite value of the cut-off $\Gamma$ ) and the "dressed" $\Gamma$ "physical" or "renormalized" parameters that are defined $\Gamma$ i.e. measured Cby actual experiments. If one takes the limit $a \rightarrow 0$ The bare parameters lose any physical meaning and one must carefully define the renormalized ones (one says to choose a prescription). If one is happy of keeping the cut-off small but finite one is allowed to identify the renormalized and the bare parameters Cbecause these can now be measured. This is the approach one uses on the lattice and it corresponds to the Kadanoff-Wilson approach to renormalization.

One can write a RGE both for the bare parameters (as function of the cut-off) or Tequivalently for the renormalized ones (as function of the renormalization scale $\mathrm{i}$ i.e. the scale at which one performs the measurements). The two equations are formally identical at first order in pertubation theory $y_{1}^{19}$. This is not surprising because one can always define the renormalized coupling at a scale $\bar{a}$ to be equivalent to the bare coupling with a cut-off $a=\bar{a}$.

In any practical lattice simulation one computes the regularized integrals $\Gamma$ eq. $(\overline{5} \overline{3}(\overline{3}) \Gamma$ for a finite $a \Gamma$ using the Monte Carlo technique. The coupling constant is an input parameter that fixes the scale of the problem. Relating the values of the coupling constants at different lattice spacings is $\Gamma$ in general $\Gamma$ a fine tuning problem.

\subsection{Improving the convergence}

It has been shown by Symanzik [i20] that it is possible to improve the convergence of the correlation functions of a regularized theory to its continuum limit $(a \rightarrow 0)$ from $O(a)$ to $O\left(a^{n+1}\right)$. In order to achieve this $\Gamma i t$ is necessary to add to the action $\mathcal{S}_{\mathrm{E}}$ terms which are proportional to $a, a^{2}, \ldots, a^{n}$ and adjust the corresponding coefficients. These tersm are called irrelevant operators

\footnotetext{
${ }^{9}$ Only at second order in perturbation theory the RGE for the renormalized parameters shows a dependence from their exact definition, the renormalization prescription.
} 
since their contribution vanishes in the limit $a \rightarrow 0$. This improvement technique is heavily used in lattice simulations where the minimum length scale $\Gamma$ the lattice spacing $a$ Ccannot be reduced arbitrarily dependence on $a$ of the correlation functions to be as small as possible.

\subsection{Lattice regularization and momentum cut-off}

We have shown as any given smooth field $\phi(x)$ defined in $[0, L]$ can be approximated with $\delta(a, x)$ functions

$$
\phi(x) \simeq \phi^{\mathrm{latt}}(a, x) \stackrel{\text { def }}{=} \sum_{k=0}^{K-1} \phi_{k} \delta(a, x-k a)
$$

with $K=L / a$.

The same field $\phi$ expanded in Fourier components

$$
\phi(x)=\sum_{n=0}^{\infty} b_{n} e^{i p_{n} x}
$$

where

$$
p_{n} \stackrel{\text { def }}{=} \frac{2 \pi n}{L} ; \quad b_{n} \stackrel{\text { def }}{=} \frac{1}{2 \pi} \int_{0}^{L} \phi(x) e^{-i p_{n} x} d x
$$

Also the right hand side of eq.( $(1-\overline{6})$ can be expanded in Fourier components and this can be written as

$$
\phi^{\text {latt }}(a, x)=\sum_{n=0}^{\infty} b_{n}^{\prime} e^{i p_{n} x}
$$

where

$$
b_{n}^{\prime}=\frac{1}{2 \pi} \sum_{k=0}^{K-1}\left[\phi_{k} \int_{0}^{L} \delta(a, k a-x) e^{-i p_{n} x} d x\right]
$$

It becomes evident that for $p_{n}>1 / a$ the integrand oscillates fast and the corresponding integral $\Gamma b_{n}^{\prime}$ Thas to be small; while for $p_{n}<1 / a$ the integral is almost constant and approximately equal to $e^{-i p_{n} k a} \Gamma$ therefore $b_{n}^{\prime} \simeq b_{n}$. The 

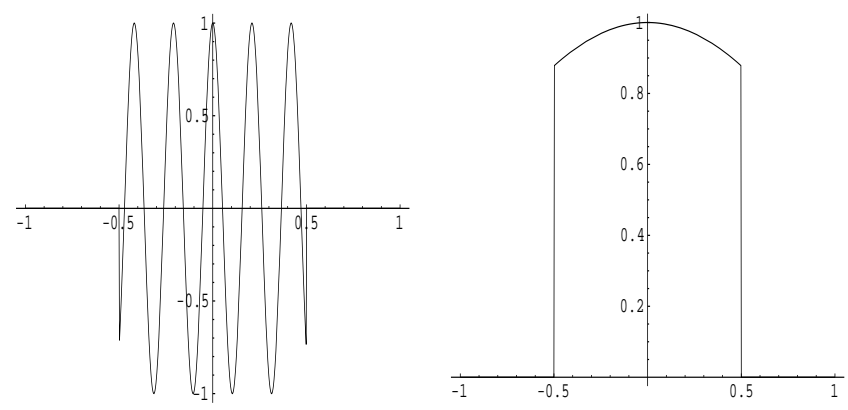

Figure 10: Different behavior of the integrand of $b_{n}^{\prime}$ for high frequency modes (left) and low frequency modes (right) respectively. The $x$ axis is in units of $x / L$.

different behavior of the integrand is shown in figure $1 \overline{0}$. This proves that eq. $(1-\overline{6} \overline{6}-1)$ can be written as

$$
\phi(x) \simeq \phi^{\mathrm{latt}}(a, x) \simeq \phi^{\mathrm{co}}(a, x) \stackrel{\text { def }}{=} \sum_{n=0}^{\infty} \theta\left(\frac{1}{a}-p_{n}\right) b_{n} e^{i p_{n} x}
$$

If $\phi(x)$ describes some physical quantity and one has a finite resolution in space $\bar{a} \Gamma$ then $\phi(x)$ can be replaced by its Fourier expansion with a cut-

off in momentum space $p_{\max }<\bar{a}^{-1}$. Therefore a momentum cut-off can be regarded as an alternative procedure to regularize the path integral.

Note that $b_{0}$ is the mean value of $\phi(x)$. Moreover $p_{1}=2 \pi / L$ represents the minimum energy/momentum mode that can propagate on a finite unidimensional volume of length $L$.

The superscripts "latt" and "co" Tused to identify the two different regularizations Tare abbreviations of lattice and cut-off respectively.

\subsection{Remarks on the physics of Effective Theories}

We have seen how Tin QFT Tone is forced to introduce three different length scales:

- $\bar{a}$ the higher spatial (temporal) resolution of current experiments.

- $a$ : the scale that corresponds to the precision of the mathematical descriptionTthe cut-off in the Kadanoff-Wilson approach to Renormalization Group. The theory is said to be renormalizable if this scale 
can be arbitrarily small. This does not mean that one can trust the predictions of the theory with arbitrary precision.

- $1 / \Lambda$ : the typical lenght scale of the physics being studied. This scale is in nature and there is no freedom to fix it. For QCD it is $\Lambda_{\mathrm{QCD}} \simeq$ $250 \mathrm{MeV}\left(1 / \Lambda_{\mathrm{QCD}} \simeq 1 \mathrm{fm}\right)$.

It is usually possible to model the same phenomenon using different QFTs $\Gamma$ which differ in the regularization-renormalization prescription and/or the renormalization scale. The predictions of these different theories must be compatible with each other apart from order $O(a)$ corrections.

Some QFTs have a finite number of coupling constants and it is possible to give a well defined meaning to the limit $a \rightarrow 0$ because all the possible divergencies can be absorbed in the renormalized constants. These QFTs are said to be renormalizable. Other QFTs are not renormalizable because it is not possible to absorb all the divergencies in a finite set of constants. The possible correlation functions $\Gamma$ at different orders in perturbation theory Texhibit an infinite variety of divergent behaviour. Originally it was believed that only renormalizable QFT made sense ${ }_{-}^{\text {IT. }}$. The modern picture is different: if the world is described by a continuous QFT Tit must be a renormalizable one. But the world could have a minimum length scale and the renormalization requirement is no longer a fundamental one. Perhaps the most important modern interpretation of these results is that $\Gamma$ if one wants to formulate a QFT to describe physics down to a finite resolution $\Gamma \bar{a} \Gamma$ and one does not pretend it to be the theory of everything Tit does not have to be renormalizable (because one does not pretend to send the scale $a$ to zero) Hi particular kind of theories are called effective theories: ${ }^{\prime 1} !$.

In the real world $\Gamma$ there might be new supersymmetric interactions or superstring $\Gamma$ or electrons and muons may have internal stucture $\Gamma$ none of which is incorporated in the Standard Model. Nevertheless the Standard Model $\Gamma$ as it is today explains the results of our experiments with a typical accuracy of $10 \%$. Renormalization saves us by saying that one does not need to know what happens at short distance (high momenta) in order to understand low energy experiments.

\footnotetext{
${ }^{10}$ This requirement led Weinberg, Glashow and Salam to formulate the Standard Model.

${ }^{11}$ E.g. the Fermi theory of electroweak interactions is not renormalizable but it is able to describe with good accuracy weak interactions at energy scales below $m_{W}$
} 
Since we will never be able to probe the physical world at every length scale every quantum field theory should be considered an effective theory. 


\section{Lattice QCD}

He who can properly define and divide is to be considered a god

(Plato)

At the typical hadronic scale while electroweak effects can be computed in the standard perturbative wayTQCD effects are not. Therefore it becomes necessary to formulate QCD in such a way to make it possible to perform a numerical computation of the correlation functions.

To reach this goal we need to regularize the theory on a lattice by introducing a finite lattice spacing $a$ and write down the QCD action in terms of the discretized fields.

In this section we will present one possible discretization of the action (due to Wilson) and the problems connected with the definition of chiral (massless) fermionic fields on the lattice.

In principle one can compute the correlation functions of QCD with arbitrary precision by reducing the lattice spacing $a$. In practice one only has a finite computational power therefore one cannot arbitrarily reduce $a$. Hence we will show how to "correct" the action to improve the correction of the correlation functions from order $O(a)$ to order $O\left(a^{2}\right)$.

We will then discussed the errors associated with the typical lattice approximations.

In the end we will present $\Gamma$ as an example $\Gamma$ a full program to compute $f_{B} \sqrt{m_{B}}$ and some recent lattice results for this quantity.

\subsection{Basic degrees of freedom and action}

The Aharonov-Bohm experiment revealed that the gauge field $A_{\mu}=t^{a} A_{\mu}^{a}$ is not observable because it is gauge dependent Tbut the phase of a particle in a gauge field background $\Gamma$ moving from $x$ to $y$ Tis an observable

$$
\mathcal{P} e^{i g \int_{x}^{y} A_{\mu} \mathrm{d} x^{\mu}}
$$

$(\mathcal{P}$ indicates a path-ordered exponential). 
For a finite lattice spacing $a$ Tit becomes convenient to write the action of QCD in terms of the shortest paths on the lattice

$$
\begin{aligned}
U_{\mu}(x) & \stackrel{\text { def }}{=} e^{i g \int_{x}^{x+a \mu} A_{\mu} \mathrm{d} x^{\mu}} \simeq 1+i g a A_{\mu}(x+a \widehat{\mu} / 2) \\
U_{-\mu}(x) & \stackrel{\text { def }}{=} e^{i g \int_{x}^{x-a \mu} A_{\mu} \mathrm{d} x^{\mu}}=U_{\mu}^{\dagger}(x-a \widehat{\mu}) \simeq 1-i g a A_{\mu}(x-a \widehat{\mu} / 2)
\end{aligned}
$$

(which are associated to the straight path connecting two consecutive lattice sites). In QCD $U$ are $S U(3)$ matrices $\Gamma$ called links.

The fermionic degrees of freedom instead $\Gamma$ the quarks $\Gamma$ can naively be defined as $q_{\alpha}^{i}(x)$ fields associated to the lattice sites $x$ (where $\alpha$ is the spin index and $i$ is the color index).

The basic discretized operators which appear in the Lagrangian $\Gamma$ are:

- Ordinary derivative $\mathbf{1}_{1:}^{\mathrm{i} 2}$

$$
\partial_{\mu} q(x) \stackrel{\text { def }}{=} \frac{1}{2 a}[q(x+a \widehat{\mu})-q(x-a \widehat{\mu})]
$$

- Covariant derivative:

$$
D_{\mu} q(x) \stackrel{\text { def }}{=} \frac{1}{2 a}\left[U_{\mu}(x) q(x+a \widehat{\mu})-U_{-\mu}(x) q(x-a \widehat{\mu})\right]
$$

It is trivial to check that in the continuum limit it is equivalent to the usual covariant derivative of QCD. In fact $\Gamma$ up to order $a$ corrections $\Gamma$

$$
\begin{aligned}
D_{\mu} q(x)= & \frac{1}{2 a}\left[\left(1+i g a A_{\mu}(x)+\ldots\right)\left(1+a \partial_{\mu}+\ldots\right) q(x)-\right. \\
& \left.\left(1-i g a A_{\mu}(x)+\ldots\right)\left(1-a \partial_{\mu}+\ldots\right) q(x)\right] \\
= & \left(\partial_{\mu}+i g A_{\mu}(x)+\ldots\right) q(x)
\end{aligned}
$$

For practical purposes That will be examined below $T$ one is usually interested in the Euclidean formulation of Lattice QCD. This is achieved by performing the Wick rotation! $n_{!}^{13 !}$

$$
x_{0} \rightarrow i x_{0} ; \quad x_{i} \rightarrow x_{i}
$$

\footnotetext{
${ }^{12}$ We remark that a different choice could be made as long as in the limit $a \rightarrow 0$ one recovers the ordinary derivative.

${ }^{13}$ The way how different quantities transform under the Wick rotation is reported in table $(\underline{1}-101 i)$
} 
Under this rotation the exponential term in the action becomes real

$$
e^{i \mathcal{S}}=e^{i a^{4} \sum_{x} \mathcal{L}} \rightarrow e^{-\mathcal{S}_{E}}=e^{-a^{4} \sum_{x} \mathcal{L}_{E}}
$$

From now on all the quoted quantities (including $\gamma$ matrices) will be Euclidean. In terms of the links Tany correlation function in QCD can be written as

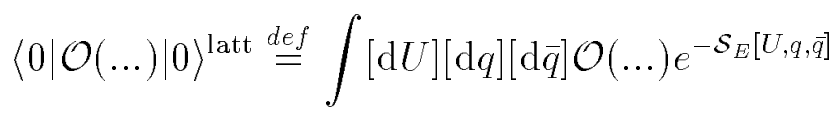

where [2521]

$$
\mathcal{S}_{\mathrm{E}}=\mathcal{S}_{\mathrm{E}}^{\text {gauge }}+\mathcal{S}_{\mathrm{E}}^{\text {quark }}
$$

and the two contributions are the gauge (pure Yang-Mills) and quark respectively. We write them in terms of the links as

$$
\begin{aligned}
\mathcal{S}_{\mathrm{E}}^{\text {gauge }} & \stackrel{\text { def }}{=} \beta \sum_{x, \mu, \nu}\left[1-\frac{1}{3} \operatorname{Re} \operatorname{tr} P_{\mu \nu}(x)\right] \\
& =a^{4} \sum_{x} \frac{1}{4} \operatorname{tr} G_{\mu \nu}^{a}(x) G^{a \mu \nu}(x)+O\left(a^{2}\right)
\end{aligned}
$$

with

$$
P_{\mu \nu} \stackrel{\text { def }}{=} U_{\mu}(x) U_{\nu}(x+a \hat{\mu}) U_{-\mu}(x+a \hat{\mu}+a \hat{\nu}) U_{-\nu}(x+a \hat{\nu})
$$

and

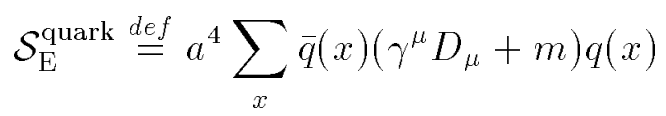

The variable $\beta=6 / g^{2}(a)$ has been introduced to conform to the standard notation of Lattice QCD. In the gauge part of the action there are no order $a$ corrections and the first corrections arise at the order $a^{2}$. On the other side $\Gamma$ in the quark part of the Lagrangian $\Gamma$ order $a$ corrections play very important role for the following two reasons:

- If one neglects order $a$ corrections and naively uses the lattice covariant derivative to implement $\mathcal{S}_{E}^{\text {quark }}$ Tone obtains a free quark propagator of the form

$$
S(p)=\frac{a}{i \gamma^{\mu} \sin \left(p_{\mu} a\right)+a m}
$$


which has 16 zeros in the Brillouin zone in the limit $m \rightarrow 0$ to be confronted with the single zero of the continuum propagator

$$
S(p)=\frac{1}{i \gamma^{\mu} p_{\mu}+m}
$$

This problem is known as doubling. To get rid of this proliferation of zero modes Wilson proposed to add to the action a term of order $a$ of the form

$$
-a^{5} \frac{r}{2} \sum_{x, \mu} \bar{q}(x) \frac{1}{a^{2}}\left[U_{\mu}(x) q(x+a \widehat{\mu})-2 q(x)+U_{-\mu}(x) q(x-a \widehat{\mu})\right]
$$

In practical simulations $r$ is fixed to be 1 nnevertheless it is convenient to show its dependence. Note that eq. $\left(\begin{array}{l}\overline{1} \\ \overline{1} \\ \overline{1}\end{array}\right)$ is a mass term therefore it explicitly breaks chiral symmery.

- When computing correlation functions one is always interested in the limit $a \rightarrow 0$ and one would like to improve the convergence of correlation functions from order $a$ to order $a^{2}$. This can be done by adding to the Action terms of order $a$ that compensate for the discretization errors up to the same order [24]. In particular one can choose a term of the form

$$
-a^{5} \frac{c_{S W}}{4} \sum_{x, \mu>\nu} \bar{q}(x) \gamma^{\mu} \gamma^{\nu} G_{\mu \nu}(x) q(x)
$$

where $G_{\mu \nu}$ is a discretized version of the chromo-electro-magntic tensor and the constant $c_{S W}$ must be fixed somehow. This term is usually referred to as clover term. It is important to observe that $c_{S W}$ is not a new free parameter of the theory $\mathrm{Cit}$ is uniquely determined by the value of $\beta$ (i.e. by the lattice spacing).

Including these $O(a)$ corrections $\Gamma$ the quark part of the action can be re-written as

$$
\mathcal{S}_{E}^{\text {quark }}[U, q, \bar{q}]=\frac{a^{3}}{2 \kappa} \sum_{y} \bar{q}(x) Q_{x y}[U] q(y)
$$


where

$$
\begin{aligned}
Q_{x y}[U]=\delta_{x y} & -\kappa \sum_{\mu}\left[\left(r-\gamma^{\mu}\right) U_{\mu}(x) \delta_{x, y-\mu}+\left(r+\gamma^{\mu}\right) U_{-\mu}(x) \delta_{x, y+\mu}\right] \\
& -\frac{\kappa c_{S W}}{2} \sum_{\mu>\nu} \gamma^{\mu} \gamma^{\nu} G_{\mu \nu}(x) \delta_{x y}
\end{aligned}
$$

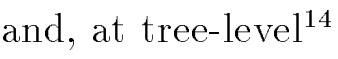

$$
\beta=\frac{6}{g^{2}(a)} ; \quad \kappa=\frac{1}{2 m a+8 r} ; \quad c_{S W}=1
$$

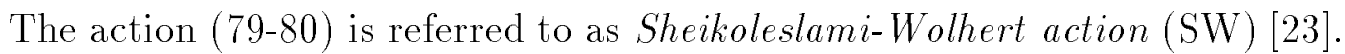

The coefficient $a^{3} / 2 \kappa$ can be absorbed in the definition of the fermionic fields $\Gamma q$ and $\bar{q}$.

In practice $\Gamma$ in lattice simulations $\Gamma a$ is not an input parameter $\Gamma$ since it is dimensionfull. Therefore one fixes the lattice spacing $\Gamma$ using dimensional transmutation $\Gamma$ by setting a value for $\beta=6 / g^{2}(a)$. One procedes in the following way:

One measures on the lattice some dimensionfull quantity $\mathrm{Tm}$ (for example the $K$ mass or the charmonium $1 P-1 S$ splitting) $\Gamma$ in adimensional units am and compares it with the experimental value $m^{\exp }$. From the comparison one determines the value of $a$ corresponding to the arbitrary input value of $\beta$. So that one adjust $a$ by fine tuning the bare $\beta$.

It is not surprising that the only way to fix the physical parameters of the theory is by comparing them with physical experiments.

On the other size $\Gamma$ the improvement coefficients $\Gamma c_{S W}$ for example $\Gamma$ and $\kappa_{\text {crit }}$ (the value of $\kappa$ associated to chiral fermions) are uniquely associated with the value of $\beta$ and can be determined theoretically.

\footnotetext{
${ }^{14}$ The fact that Lattice QCD with the action of eq. $\left({ }^{18} 0_{1}^{\prime}\right)$ is $O(a)$ improved for on-shell quantities does not simply appear form a Taylor expansion. To show the improvement it is necessary to list all dimension 5 operators, use the equations of motion to reduce some of them and absorb the contribution of the remaining two operators in the coupling constant and mass renormalization
}

$$
g^{2} \rightarrow g^{2}\left(1+b_{g} m a\right) ; \quad m \rightarrow m\left(1+b_{m} m a\right)
$$




\subsection{Simulation aspects and quenching}

First of all one can consider correlation functions that do not depend on quark fields and integrate out the quarks

$$
\begin{aligned}
\langle 0|\mathcal{O}(\ldots)| 0\rangle^{\text {latt }} & =\int[\mathrm{d} U][\mathrm{d} q][\mathrm{d} \bar{q}] \mathcal{O}(\ldots[U]) e^{-\mathcal{S}_{E}[U, q, \bar{q}]} \\
& =\int[\mathrm{d} U] \mathcal{O}(\ldots[U]) \operatorname{det} Q[U] e^{-\mathcal{S}_{E}^{\text {gauge }}[U]} \\
& =\int[\mathrm{d} U] \mathcal{O}(\ldots[U]) P[U]
\end{aligned}
$$

where

$$
P[U]=e^{-\mathcal{S}_{E}^{\text {gauge }}[U]+\ln \operatorname{det} Q[U]}
$$

In practice one neglects the contribution of $\ln \operatorname{det} Q[U]$ in the probability $P[U] \Gamma$ eq. (184i). This is called the quenched approximation. This is a very crude approximation and it breaks the unitarity of the theory. Its only motivation is the limitation in present computer power. It introduces a systematic error in the computations that has to be quantified.

The probability $P[U]$ is real (this is why Lattice computations are performed in Euclidean space) and resembles a Boltzman weight factor. Therefore standard statistical mechanics techniques can be applied.

We have seen how $\Gamma$ for a finite lattice spacing $\Gamma$ the Path Integral reduces to a well defined $K$-dimensional integral which can be computed numerically using Monte Carlo integration.

In a typical Lattice QCD simulation one discretizes the space-time on grid of about 48 sites in the temporal direction and 24 sites in each spatial direction. Therefore the computation of each correlation function correspond to the computation of a $K$-dimensional integral with $K=48 \times 24^{3}=663552$. This is quite a big integral!

Any standard lattice simulation begins with the creation of an ensemble of gauge configurations $\left\{U^{[i]}\right\}$. It is created through a Markov process $[2] \Gamma$ i.e. each configuration $U^{[i]}$ is generated from the preceding one $\Gamma U^{[i-1]} \Gamma u \operatorname{sing}$ a Monte Carlo algorithm satisfying the condition

$$
P\left(U^{[i-1]} \rightarrow U^{[i]}\right) P\left[U^{[i-1]}\right]=P\left(U^{[i]} \rightarrow U^{[i-1]}\right) P\left[U^{[i]}\right]
$$




\begin{tabular}{|llll|}
\hline State & $I^{G}$ & $J^{P C}$ & Operator $J$ \\
\hline scalar & $1^{-}$ & $0^{++}$ & $\bar{q} q^{\prime}$ \\
pseudoscalar & $1^{-}$ & $0^{++}$ & $\bar{q} \gamma^{0} q^{\prime}$ \\
& $1^{-}$ & $0^{-+}$ & $\bar{q} \gamma^{5} q^{\prime}$ \\
vector & $1^{-}$ & $0^{-+}$ & $\bar{q} \gamma^{0} \gamma^{5} q^{\prime}$ \\
& $1^{+}$ & $1^{--}$ & $\bar{q} \gamma^{\mu} q^{\prime}$ \\
axial & $1^{+}$ & $1^{--}$ & $\bar{q} \gamma^{\mu} \gamma^{0} q^{\prime}$ \\
tensor & $1^{-}$ & $1^{++}$ & $\bar{q} \gamma^{\mu} \gamma^{5} q^{\prime}$ \\
octet & $1^{+}$ & $1^{+-}$ & $\bar{q} \gamma^{\mu} \gamma^{j} q^{\prime}$ \\
& $\frac{1}{2}$ & $\frac{1}{2}^{-}$ & $\left(q^{T i} \gamma^{2} \gamma^{0} q^{j}\right)\left(\gamma^{5} q^{\prime \prime k}\right) \varepsilon_{i j k}$ \\
decuplet & $\frac{1}{2}$ & $\frac{1}{2}^{-}$ & $\left(q^{T i} \gamma^{2} \gamma^{0} \gamma^{5} q^{\prime j}\right)\left(q^{\prime \prime k}\right) \varepsilon_{i j k}$ \\
\hline
\end{tabular}

Table 1: Example of currents used on lattice and their relative quantum numbers. $q, q^{\prime}$ and $q^{\prime \prime}$ are different flavours. The superscripts $i \Gamma j$ and $k$ are color labels.

where $P\left(U \rightarrow U^{\prime}\right)$ is the probability of generating the configurations $U^{\prime}$ from the configuration $U$. An example of such an algorithm is the Metropolis Algorithm. A more sofisticate one is the heatbath algorithm.

Note that $P[U]$ depends on $\beta=6 / g^{2}(a)$ which is the parameter that fixes the lattice scale. The initial configuration $U^{[0]}$ is usually chosen to be "cold" $\Gamma$ i.e. when all its links are the identity Tor "hot" $\Gamma$ when each link is a random

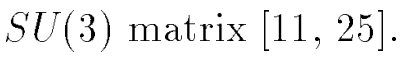

The computation of any correlation function $\Gamma$ as defined in eq. ( $(1 \overline{3} \overline{3}) \Gamma$ can be approximated $\Gamma$ in analogy with eq. $\left(\underline{2}_{2} \overline{6}_{1}^{\prime}\right) \Gamma$ as an average over the ensemble of gauge configurations

$$
\langle 0|\mathcal{O}(\ldots)| 0\rangle^{\text {latt }} \simeq \frac{1}{N} \sum_{i} \mathcal{O}\left(\ldots\left[U^{[i]}\right]\right)
$$

where $N$ is the number of generated configurations.

\subsection{Correlation functions and fermions}

The typical quantities that are measured on the lattice are the two and threepoint correlation functions between currents and their (discrete) Fourier 
transforms at zero momentum

$$
\begin{aligned}
C_{2}\left(t_{x}\right) & =\sum_{\mathbf{x}}\left\langle 0\left|J^{0}(x) J^{0 \dagger}(0)\right| 0\right\rangle \\
C_{3 \mathcal{O}}\left(t_{x}, t_{y}\right) & =\sum_{\mathbf{x}, \mathbf{y}}\left\langle 0\left|J^{0}(x) \mathcal{O}(0) J^{0 \dagger}(-y)\right| 0\right\rangle
\end{aligned}
$$

Since the lattice metric is Euclidean $\Gamma$ the asymptotic behaviour of the spatial Fourier transform of the two point correlation function $\Gamma$ in the limit $t_{x} \rightarrow \infty$ Tis given by

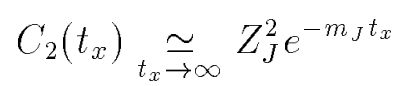

where $m_{J}$ is the mass of the lightest state $\left|1_{J}\right\rangle$ created by the current $J^{\dagger}$ and

$$
Z_{J}=\frac{\left|\left\langle 0\left|J^{0}(0)\right| 1_{J}\right\rangle\right|}{\sqrt{2 m_{J}}}
$$

From the measurement of $C_{2}\left(t_{x}\right)$ and its fit to $\left(\begin{array}{l}89 \\ 1\end{array}\right)$ it is possible to extract masses of particles $\Gamma m_{J}$. In the same fashion from the asymptotic behaviour of the ratio between the three and two-point correlation functions it is possible to extract matrix elements [i2 $\overline{6}$ i]

$$
\frac{C_{3 \mathcal{O}}\left(t_{x}, t_{y}\right)}{C_{2}\left(t_{x}\right) C_{2}\left(t_{x}\right)} \underset{t_{x}, t_{y} \rightarrow \infty}{\simeq} \frac{1}{Z_{J}^{2}} \frac{\left\langle 1_{J}|\mathcal{O}| 1_{J}\right\rangle}{2 m_{J}}
$$

The most general current $J^{\mu}(x)$ is expressed in terms of fundamental fermionic fields $q_{\alpha}^{i}(x)$ (the quark fields). A list of some interesting currents

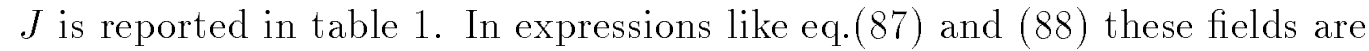
Wick contracted

$$
S_{a b}^{i j}(x, y) \stackrel{\text { def }}{=}\left\langle 0\left|\left\{q_{a}^{i}(x), \bar{q}_{b}^{j}(y)\right\}\right| 0\right\rangle
$$

Despite the fact that fermions are neglected when gauge configurations are created $\Gamma$ they are re-introduced at a later stage as particles propagating in the gluonic background field. Therefore the two and three point correlation functions can be written as appropriate traces of propagators $\Gamma S_{a b}^{i j}(x, y,[U]) \Gamma$ in the backgroud gluonic field $U$. For example the propagator of a heavy-light 
pseudoscalar meson (associated to the current $J^{\mu}=\bar{h}^{i} \gamma^{\mu} \gamma^{5} q^{i} \Gamma$ where $h \Gamma q$ are the heavy and the light quark respectively) Ifrom 0 to $x$ can be computed as

$$
\begin{aligned}
\left\langle 0\left|J^{0}(x) J^{0 \dagger}(0)\right| 0\right\rangle & =\left\langle 0\left|\bar{h}_{c}^{i}(x)\left(\gamma^{0} \gamma^{5}\right)^{c a} q_{a}^{i}(x) \bar{q}_{b}^{j}(0)\left(\gamma^{0} \gamma^{5}\right)^{b d} h_{d}^{j}(0)\right| 0\right\rangle \\
& =\left\langle 0\left|S_{q a b}^{i j}(x, 0)\left(\gamma^{0} \gamma^{5}\right)^{c a} S_{h d c}^{j i}(0, x)\left(\gamma^{0} \gamma^{5}\right)^{b d}\right| 0\right\rangle \\
& =\frac{1}{N} \sum_{\{U\}} S_{q a b}^{i j}(x, 0,[U])\left(\gamma^{0} \gamma^{5}\right)^{c a} S_{h d c}^{j i}(0, x,[U])\left(\gamma^{0} \gamma^{5}\right)^{b d}
\end{aligned}
$$

( $i \Gamma j$ are color indices and $a \Gamma b \Gamma c \Gamma d$ are spin indices). Then by making use of the $\mathrm{H}$ discrete symmetry (see Appendix A) and properties of the $\gamma$ matrices one obtains

$$
\left\langle 0\left|J^{0}(x) J^{0 \dagger}(0)\right| 0\right\rangle=\frac{1}{N} \sum_{\{U\}} \operatorname{tr}\left\{S_{q a b}(x, 0,[U]) S_{h a b}^{\dagger}(x, 0,[U])\right\}
$$

(the trace is only on the color indices $i$ and $j$ ).

On each gauge configuration $U \Gamma$ the fermion propagator $S$ is computed by inverting numericaly the fermionic matrix

$$
S(x, y,[U])=(Q[U])_{x y}^{-1}
$$

This is the most expensive part of any lattice calculations. In the computation of the propagator $\kappa$ and $c_{S W}$ are input parameters. The former is in one to one correspondence with the fermion mass (the pole-mass)

$$
m=\frac{1}{a} \ln \left(1+\frac{1}{2}\left(\frac{1}{\kappa}-\frac{1}{\kappa_{\text {crit }}}\right)\right)
$$

and $\kappa_{\text {crit }}$ is a parameter depending on $\beta$. The chiral limit corresponds to the limit $\kappa \rightarrow \kappa_{\text {crit }} \Gamma$ when the quark becames massless. In practice any inversion algorithm for eq. $\left(\overline{0} \overline{p_{1}^{\prime}}\right)$ converges slower and slower as the chiral limit is approached and this can never be reached.

There are two standard ways of computing the fermion propagator: exact and stochastic. The former is very time expensive therefore it is usual normal practice to compute propagators ending in one single point of the lattice. The latter is less precise but allows one to compute propagators from each point to each point of the lattice in a feasible time. 


\subsection{Lattice errors}

Numerical simulations of Lattice QCD are characterized by a number of statistical and systematical errors which will have to be taken into account when quoting lattice results. What follows is a list of the most common errors one has to consider $\Gamma$ possibly reduce and $\mathrm{Thopefully \Gamma quantify:}$

- Discretization errors $a$. Physical results are extracted from lattice in the limit of $a \rightarrow 0$. This limit cannot be reached in real lattice simulation and in practice one performs simulations with a finite (as small as possible) lattice spacing. The discrepancy between the computed correlation functions and their continuum limit is usually of the order of $a$ (or $a^{2}$ for improved lattice actions). In typical simulations $1 / a=1 \div 3 \mathrm{GeV}$.

- Statistical errors. All Monte Carlo simulations are based on statistical sampling therefore they introduce a statistical error that is expected to decrease with $1 / \sqrt{N}$ where $N$ is the number of independent measurements (in case of one measurement for gauge configuration $N$ is the number of uncorrelated gauge configurations). Since the gauge configurations are created using a Markov chain based on small changes to link variables $\Gamma$ one may be worried about correlations among the different configurations. Because of modern day computational power it is possible to generate reasonable statistical samples and this is no more a major problem.

- Finite volume. Because of the finite volumeTperiodic or anti-periodic boundary conditions are imposed for the field. Therefore every observable which is computed on the lattice suffers from an unphysical contri-

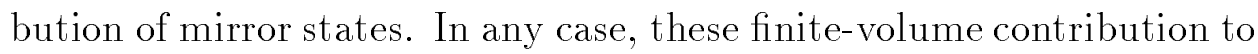
the correlation functions falls off exponentially with the lattice length and they are usually negligible for a lattice size $L$ bigger than $5 / m_{\pi}$. On the other side the effects of mirror states are crucial in preventing

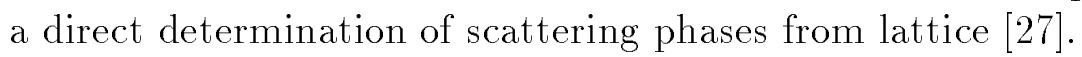

- Quenching. This approximation is the hardest to justify. Its only reason is the present limitation in computational power. As a consolation one can argue that present exploratory unquenched simulations suggest that the effects of the quark loops in the mass spectrum are 
small $\mathrm{but}$ the error introduced by quenching in the determination of $a^{-1}$ can be as big as $10 \%-20 \%$.

- Chiral extrapolation. It has been shown that because of the finite volume effects Tan infrared cut-off is naturally associated with the lattice $\Gamma$ therefore the $u$ and $d$ quarks are too light to be simulated Teven by modern day computers. Therefore one usually performs lattice simulations for values of $m_{u}=m_{d}$ much bigger of the physical values (typically bigger than $50 \mathrm{MeV}$ ) $\Gamma$ then performs an extrapolation of the results to the limit $m_{u}=m_{d}=0$. This extrapolation is called the chiral extrapolation. It corresponds to the limit $\kappa \rightarrow \kappa_{\text {crit }}$ For the masses of light particles (the pseudo-Goldstone boson) this extrapolation is guided by predictions of the Chiral Lagrangian such as the Gell-MannOkubo formula.

- Heavy quarks. The $c$ and $b$ quarks are very heavy therefore not all of their modes can propagate on a typical lattice. To solve the problem there are three common approaches. One possibility is to simulate these quarks with a mass smaller than the physical one and then to perform an extrapolation to the physical mass (guided by the Heavy Quark Effective Theory). The second possibility is to implement the HQET on lattice. This implies that one considers the heavy quark as static (non-relativistic) and $\Gamma$ in principle $\Gamma$ systematically computes corrections to this approximation in the $1 / m_{h}$ expansion. The third approach is also based on the HQET and is explained in ref. [i2 $-\overline{8} \overline{1}$.

- Matching between lattice and continuum scheme. Experimental data are analyzed using some continuum renormalization scheme $\Gamma$ usually dimensional regularization with the $\overline{\mathrm{MS}}$ prescription. To confront Lattice QCD results with phenomenology it is therefore necessary to match the matrix elements between the two different schemes. In general

$$
\begin{aligned}
\left\langle 0\left|\mathcal{O}_{i}(\ldots)\right| 0\right\rangle^{\overline{\mathrm{MS}}} & =Z_{i j}\left\langle 0\left|\mathcal{O}_{j}(\ldots)\right| 0\right\rangle^{\text {latt }} \\
& =\left(\delta_{i j}+O\left(\alpha_{s}\right)\right)\left\langle 0\left|\mathcal{O}_{j}(\ldots)\right| 0\right\rangle^{\text {latt }}
\end{aligned}
$$

where $Z_{i j}$ are called matching coefficients and have a perturbative origin. The matching coefficients can be computed in perturbation theory and usually they are known only at 1-loop. Since $\alpha_{s}$ is big at the typical 
lattice energy scale $\Gamma$ corrections of higher order in $\alpha_{s}$ can contribute to an error in the matching of as much as $10 \%$. Moreover in the matching procedure it is common that matrix elements of some continuum operator mix with the corresponding matrix elements of new operators that appear on lattice $\Gamma$ because $Z_{i j}$ is $\Gamma$ in general $\Gamma$ non diagonal. The contribution of these operators can be big and must be taken into account.

\subsection{One full application, $f_{B} \sqrt{m_{B}}$}

We have seen in section 1 how one could extract $f_{B} \sqrt{m_{B}}$ by computing the Path Integral on the right-hand side of eq. (1. $\left.\bar{A}_{1}\right) \Gamma$ the 2-point correlation function Tand fitting the result with

$$
C_{2}(t) \underset{t \rightarrow \infty}{=} \frac{f_{B}^{2} m_{B}}{2} e^{-m_{B} t}
$$

Program C2.C in the Appendix is an example of a real QCD program (written in $\mathrm{C++}$ ) that computes $C_{2}(t)$. It is based on the mathematical library described in ref.

This program is organized in the following way: It opens the libraries and declares the variable containing the parameters of the lattice simulation:

- beta $\equiv \beta$ Tthat fixes the size of the lattice spacing;

- $\mathrm{mq} \equiv m_{q}$ Tthe pole mass of the light quark;

- $\mathrm{mh} \equiv m_{h}$ Tthe pole mass of the heavy quark;

- gridsizeTthe lattice size (for example $16 \times 6^{3}$ );

- lattice Tthe object that contains the grid and its properties (including the functions to move on the lattice $\Gamma$ a local random number generator and functions necessary for the parallelization).

It then defines the basic fields:

- $\mathrm{U}(\mathrm{x}, \mathrm{mu})(\mathrm{i} j) \equiv U_{\mu}^{i j}(x) \Gamma$ the gauge field configuration;

- $\mathrm{Sq}(\mathrm{x}, \mathrm{a}, \mathrm{b})(\mathrm{i} j) \equiv\left\langle 0\left|\left\{q_{a}^{i}(x), \bar{q}_{b}^{j}(0)\right\}\right| 0\right\rangle \Gamma$ the light quark propagator;

- $\operatorname{Sh}(\mathrm{x}, \mathrm{a}, \mathrm{b})(\mathrm{i} j) \equiv\left\langle 0\left|\left\{h_{a}^{i}(x), \bar{h}_{b}^{j}(0)\right\}\right| 0\right\rangle \Gamma$ the heavy quark propagator; 
(they are fields of $3 \times 3$ color matrices and $a$ and $b$ are spin indices).

The program starts with a hot configuration (set_hot (U)) then computes and discards the first 100 gauge configurations of the Markov chain. The Heatbath (heatbath (U)) algorithm is used to generate the Markov chain. It is equivalent but more sofisticate than the Metropolis algorithm.

Then Teach 10 gauge configurations of the chain $\Gamma$ the program computes the light and heavy propagator and measures the 2-point correlation function

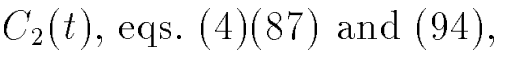

$$
\sum_{\mathbf{x}} J^{0}(x) J^{0 \dagger}(0)=\sum_{\mathbf{x}} \operatorname{tr}\left\{S_{q a b}(x, 0,[U]) S_{h a b}^{\dagger}(x, 0,[U])\right\}
$$

$\left(t=x_{0}\right.$ is kept fixed in the sum).

This is done in the following piece of code:

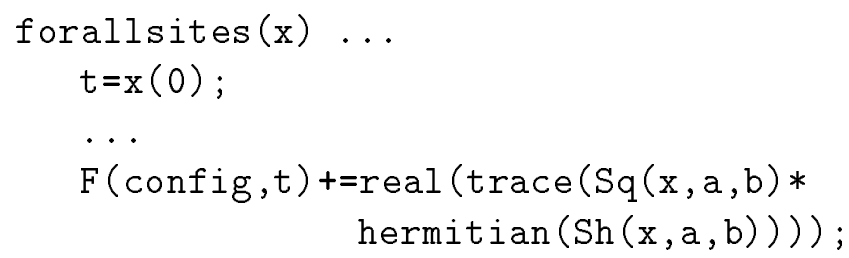

Note that the average of the trace in question is always real by virtue of the theorem of Appendix A (applied to the case $\vec{p}=0$ ). This is in agreement with naive expectations from eq. (㐬充).

Finally the program computes the average over the gauge configurations of $C_{2}(t)$ (with its the Bootstrap error) and prints out the results.

The output of the program is plotted in fig. I for some arbitrary input values of the parameters. In this example $i$ in fact we did not attempt to tune the parameters properly since our main concern was to have a fast running program for didactic purposes.

For a real state-of-the-art computation of $f_{B} \sqrt{m_{B}}$ we refer now to ref. The program used in that simulation is very similar to the one discussed here. The only operative differences are in the choice of the parameters and in the

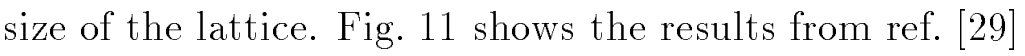

The values of $f_{B} \sqrt{m_{B}}$ are computed by fitting $C_{2}(t)$ for different sets of input parameters $m_{q}$ and $\beta \Gamma$ while $m_{h}$ is tuned to the $B$ quark pole-mass. These results are first extrapolated to $m_{q} \rightarrow 0$ and then extrapolated to $a \rightarrow 0(\beta \rightarrow \infty)$.

The results obtained in this way are renormalized in the lattice scheme at the lattice energy scale $a^{-1}$. To obtain the numbers renormalized in the 
$\overline{\mathrm{MS}}$ scheme at the $m_{B}$ energy scale they need to be corrected by a matching factor.

In the paper in exam the lattice spacing $a$ is measured (as function of $\beta$ ) by confronting the numerical result for the $1 \mathrm{P}-1 \mathrm{~S}$ mass splitting in the charmonium spectrum with experimental results. 

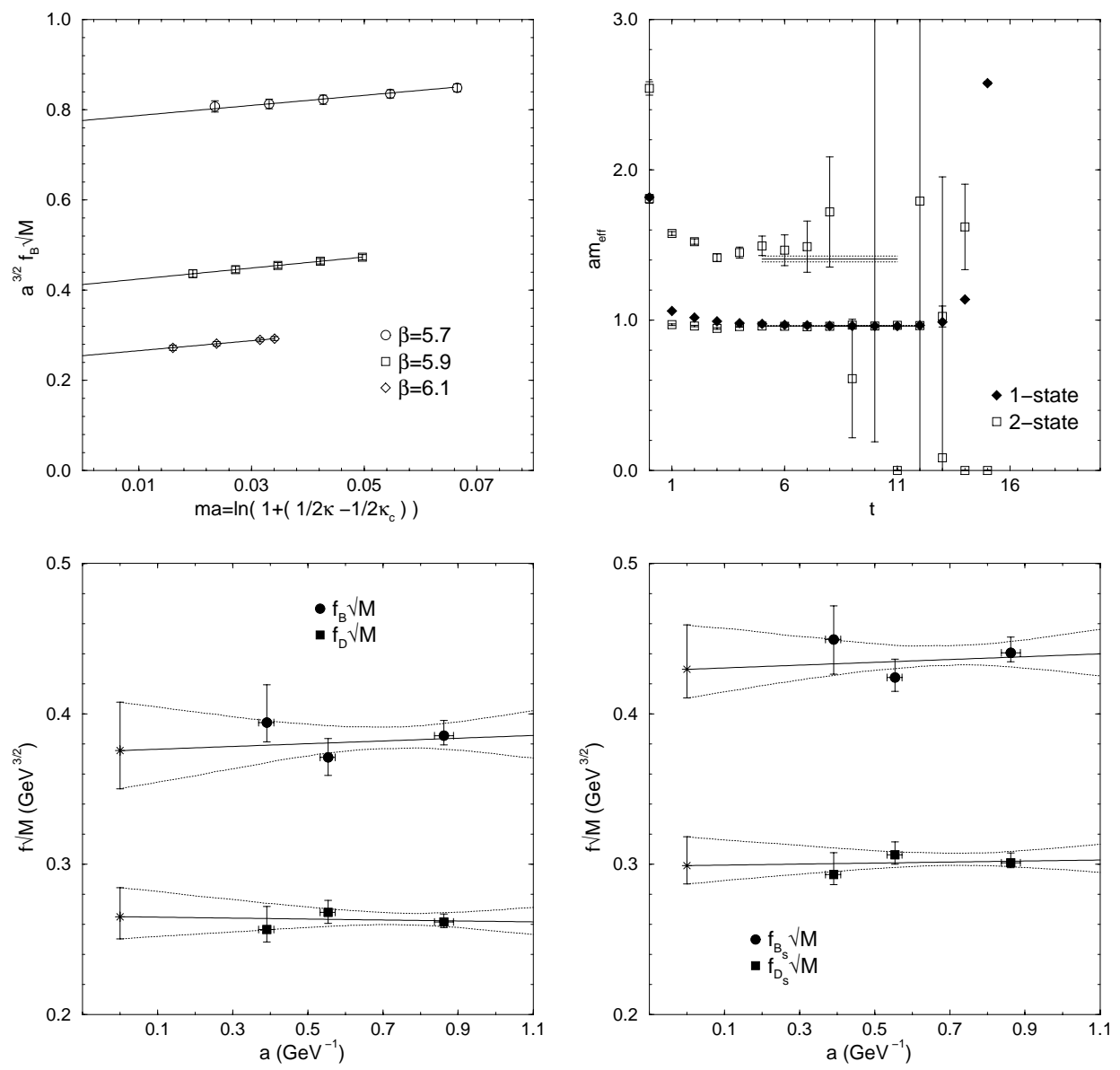

Figure 11: Determination of $f_{B} \sqrt{m_{B}} \Gamma f_{D} \sqrt{m_{D}} \Gamma f_{B_{s}} \sqrt{m_{B_{s}}}$ and $f_{D_{s}} \sqrt{m_{D_{s}}}$ (Fer-

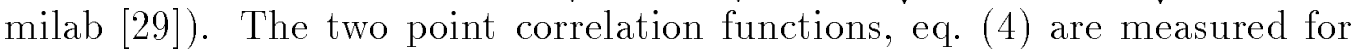
different values of the lattice spacing $a$ and different masses for the light quarks $m=1 / a \ln \left(1+1 /(2 \kappa)-1 /\left(2 \kappa_{c}\right)\right)$ and fitted to extract $f_{B} \sqrt{m_{B}}$. The results are extrapolated to the chiral limit $m \rightarrow 0$ (top-left). Then the lattice spacing is determined by measuring the $1 \mathrm{P}-1 \mathrm{~S}$ mass splitting in the charmonium spectrum (top-right). The chirally extrapolated values for $f_{B} \sqrt{m_{B}} \Gamma$ $f_{D} \sqrt{m_{D}} \Gamma f_{B_{s}} \sqrt{m_{B_{s}}}$ and $f_{D_{s}} \sqrt{m_{D_{s}}}$ are extrapolated to the continuum limit $a \rightarrow 0$ (bottom-left and bottom-right). 


\section{A Euclidean Space-Time in $d=4$ dimension}

\section{A.1 Wick rotation}

The Euclidean action is obtained from the Minkowskian one by performing a Wick rotation. Under this rotation the basic vectors of the theory transform according with the following table (E for Euclidean $\Gamma \mathrm{M}$ for Minkowski)

\begin{tabular}{|cc|cc|}
\hline $\mathrm{E}$ & $\mathrm{M}$ & $\mathrm{E}$ & $\mathrm{M}$ \\
\hline$x^{0}$ & $i x^{0}$ & $x^{i}$ & $x^{i}$ \\
$\partial^{0}$ & $-i \partial_{0}$ & $\partial^{i}$ & $\partial_{i}$ \\
$A^{4}$ & $-i A_{0}$ & $A^{i}$ & $A_{i}$ \\
$F^{0} i$ & $-i F_{0 i}$ & $F^{i j}$ & $F_{i j}$ \\
$\gamma^{0}$ & $\gamma^{0}$ & $\gamma^{i}$ & $-i \gamma^{i}$ \\
$\gamma^{5}$ & $\gamma^{5}$ & & \\
\hline
\end{tabular}

and the integration measure transforms as follow

$$
\exp \left(-\mathcal{S}_{E}\right)=\exp \left(i \mathcal{S}_{M}\right)
$$

where

$$
\mathcal{S}_{E}=\int \mathrm{d}^{4} x_{E} \mathcal{L}_{E}[\ldots]=-i \int \mathrm{d}^{4} x_{M} \mathcal{L}_{M}[\ldots]
$$

The choice $\mathrm{d}^{4} x_{E}=i \mathrm{~d}^{4} x_{M}$ can be made hhence $\mathcal{L}_{E}[\ldots]=-\mathcal{L}_{M}[\ldots]$

The Euclidean metric tensor is defined as

$$
g_{E}^{\mu \nu}=-\delta^{\mu \nu}=\operatorname{diag}(-1,-1,-1,-1)
$$

\section{A.2 Spin matrices}

- Dirac matrices (Dirac representation)

$$
\gamma^{0}=\left(\begin{array}{ll}
\mathbf{1} & 0 \\
0 & -\mathbf{1}
\end{array}\right) \Gamma \quad \gamma^{i}=\left(\begin{array}{ll}
0 & -i \sigma_{i} \\
i \sigma_{i} & 0
\end{array}\right) \Gamma \quad \gamma^{5}=\left(\begin{array}{ll}
0 & 1 \\
1 & 0
\end{array}\right)
$$

- Dirac matrices (Chiral representation)

$$
\gamma^{0}=\left(\begin{array}{ll}
0 & \mathbf{1} \\
\mathbf{1} & 0
\end{array}\right) \Gamma \quad \gamma^{i}=\left(\begin{array}{ll}
0 & -i \sigma_{i} \\
i \sigma_{i} & 0
\end{array}\right) \Gamma \quad \gamma^{5}=\left(\begin{array}{ll}
-\mathbf{1} & 0 \\
0 & \mathbf{1}
\end{array}\right)
$$


All the Euclidean Dirac matrices are hermitian. The following relations hold

$$
\begin{aligned}
g^{\mu \nu} & =\frac{1}{2}\left\{\gamma^{\mu}, \gamma^{\nu}\right\}=\delta^{\mu \nu} \\
\sigma^{\mu \nu} & =\frac{i}{2}\left[\gamma^{\mu}, \gamma^{\nu}\right] \\
\gamma^{5} & =\gamma^{0} \gamma^{1} \gamma^{2} \gamma^{3}
\end{aligned}
$$

and all the $\sigma^{\mu \nu}$ are hermitian.

- Projectors

$$
L=\frac{1-\gamma^{5}}{2} \quad R=\frac{1+\gamma^{5}}{2}
$$

- Traces

$$
\begin{aligned}
\operatorname{tr}\left(\gamma^{\mu} \gamma^{\nu}\right) & =4 \delta^{\mu \nu} \\
\operatorname{tr}\left(\gamma^{\mu} \gamma^{\nu} \gamma^{\rho}\right) & =0 \\
\operatorname{tr}\left(\gamma^{\mu} \gamma^{\nu} \gamma^{\rho} \gamma^{\sigma}\right) & =4\left(\delta^{\mu \nu} \delta^{\rho \sigma}-\delta^{\mu \rho} \delta^{\nu \sigma}+\delta^{\mu \sigma} \delta^{\rho \nu}\right) \\
\operatorname{tr}\left(\gamma^{5} \gamma^{\mu} \gamma^{\nu} \gamma^{\rho} \gamma^{\sigma}\right) & =4 \epsilon_{E}^{\mu \nu \rho \sigma}
\end{aligned}
$$

where $\epsilon_{E}^{0123}=-1$.

\section{A.3 Lattice discrete symmetries}

The lattice formulation of QCD is invariant under the following discrete symmetries of the quark propagator [3-1]

- Parity, $P$ :

$$
S_{\alpha \beta}^{i j}(x, y,[U])=\gamma_{\alpha \alpha^{\prime}}^{0} S_{\alpha^{\prime} \beta^{\prime}}^{i j}\left(x^{P}, y^{P},\left[U^{P}\right]\right) \gamma_{\beta^{\prime} \beta}^{0}
$$

- Charge conjugation, $C$ :

$$
S_{\alpha \beta}^{i j}(x, y,[U])=\left(\gamma^{0} \gamma^{2}\right)_{\alpha \alpha^{\prime}} S_{\alpha^{\prime} \beta^{\prime}}^{j i}\left(y, x,\left[U^{C}\right]\right)\left(\gamma^{2} \gamma^{0}\right)_{\beta^{\prime} \beta}
$$

- Time reversal, $T$ :

$$
S_{\alpha \beta}^{i j}(x, y,[U])=\left(\gamma^{0} \gamma^{5}\right)_{\alpha \alpha^{\prime}} S_{\alpha^{\prime} \beta^{\prime}}^{i j}\left(x^{T}, y^{T},\left[U^{T}\right]\right)\left(\gamma^{5} \gamma^{0}\right)_{\beta^{\prime} \beta}
$$




\section{- $H$ symmetry:}

$$
S_{\alpha \beta}^{i j}(x, y,[U])=\gamma_{\beta \alpha^{\prime}}^{5} S_{\alpha^{\prime} \beta^{\prime}}^{j i}(y, x,[U]) \gamma_{\beta^{\prime} \alpha}^{5}
$$

$U^{P}, U^{C}, U^{T}$ are the parity reversed $\Gamma$ charge conjugate $\Gamma$ time reversed gauge configurations respectively.

\section{A.4 Theorems about correlation functions}

These discrete symmetries play a very important role because they put contraints on the Euclidean correlation functions. In particular one can consider correlation function of the form

$$
G^{(n, m)}\left(\vec{p}_{1}, \ldots, \vec{p}_{n}\right)=\int \mathrm{d}^{4} x_{1} e^{i p_{1} x_{1}} \ldots \mathrm{d}^{4} x_{n} e^{i p_{n} x_{n}}\left\langle 0\left|\operatorname{tr}\left\{S \gamma^{\mu_{1}} S \gamma^{\mu_{2}} \ldots S \gamma^{\mu_{m}}\right\}\right| 0\right\rangle
$$

where the trace is in spin and color $\Gamma$ and $S$ are quark propagators connecting an arbitrary couple of points in the ensemble $\left\{x_{1}, \ldots, x_{n}\right\}$. Imposing invariance under $P$ (parity) Tone obtains that

$$
G^{(n, m)}\left(\vec{p}_{1}, \ldots, \vec{p}_{n}\right)=(-1)^{N} G^{(n, m)}\left(-\vec{p}_{1}, \ldots,-\vec{p}_{n}\right)
$$

where $N$ is the number of indices $\mu_{1} \Gamma$.. Г $\mu_{m}$ that differ from 0 . Eq. ( $(1 \overline{2} \overline{2} \overline{0})$ is true also in the Minkowski space.

Imposing invariance under $P C H$ one obtains that

$$
G^{(n, m)}\left(\vec{p}_{1}, \ldots, \vec{p}_{n}\right)=(-1)^{N}\left[G^{(n, m)}\left(\vec{p}_{1}, \ldots, \vec{p}_{n}\right)\right]^{*}
$$

This tells whether any correlation function is real or imaginary.

Eq. (121i) is not true in Minkowski space. It is replaced by an equivalent expression where $N$ counts the total number of indices $\mu_{1}$ Г... $\mu_{m}$ that are equal to 5 .

As an example we consider

$$
G^{(3,2)}(\vec{p})=\int \mathrm{d}^{4} x e^{i p x}\left\langle 0\left|\operatorname{tr}\left\{S(x, 0) \gamma^{1} \gamma^{5} S(0, x) \gamma^{2}\right\}\right| 0\right\rangle
$$

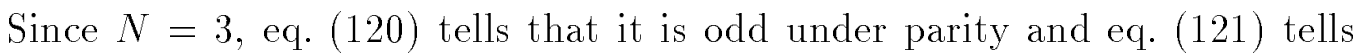
that it is imaginary. Hence for $p=0$ it must be zero (because it is odd under parity). 


\section{B Example programs}

\section{B.1 Basic integration: program1.c}

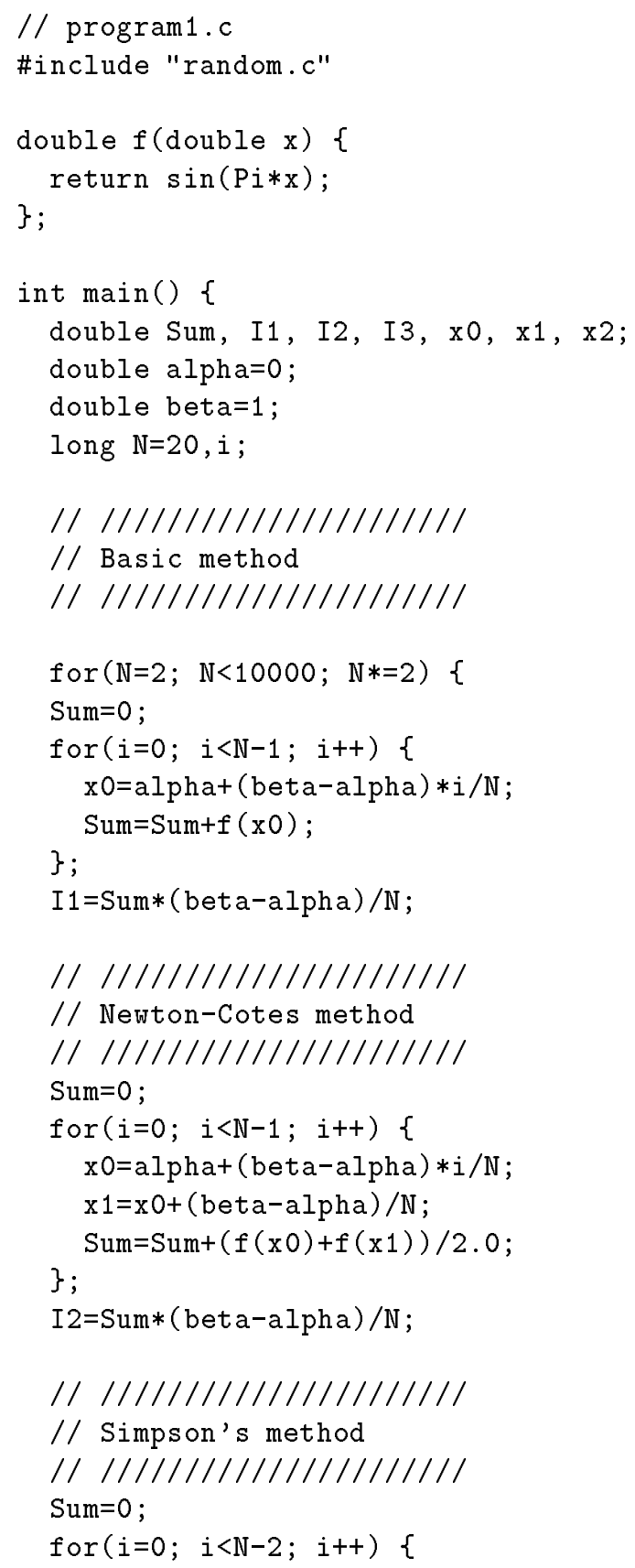




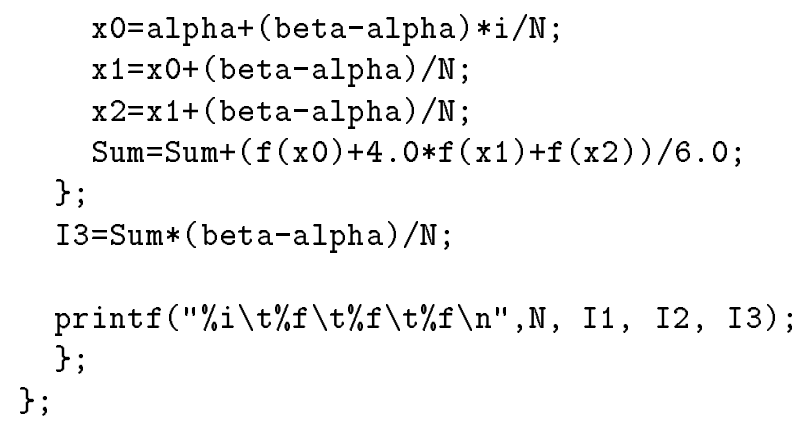

B.2 Monte Carlo integration: program2.c

// program2.c

\#include "random.c"

double f(double $x)\{$

return $\sin \left(\mathrm{P}_{i * x}\right)$;

\} ;

int $\operatorname{main}()\{$

double I, Sum, $x$;

long $\mathrm{N}$;

Sum $=0$;

for $(\mathrm{N}=1 ; ; \mathrm{N}++)\{$ $\mathrm{x}=\mathrm{Random}()$;

\section{B.3 Monte Carlo integration in 3D: program3.c}

// program3.c

\#include "random. c"

double $f($ double $* x)\{$

return $3.0 * \mathrm{x}[0] * \mathrm{x}[0] * \mathrm{x}[1]+2.0 * \mathrm{x}[2] * \mathrm{x}[2] * \mathrm{x}[2]$;

\} ;

int $\operatorname{main}()\{$

double I, Sum, $x[3]$;

long $\mathrm{N}$; 


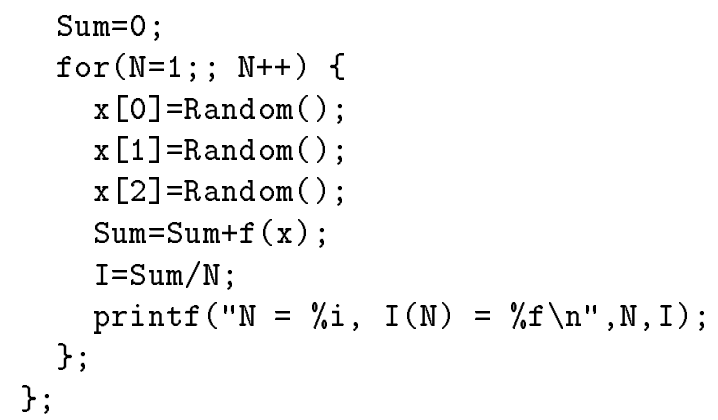

B.4 Metropolis Monte Carlo integration: program4.c

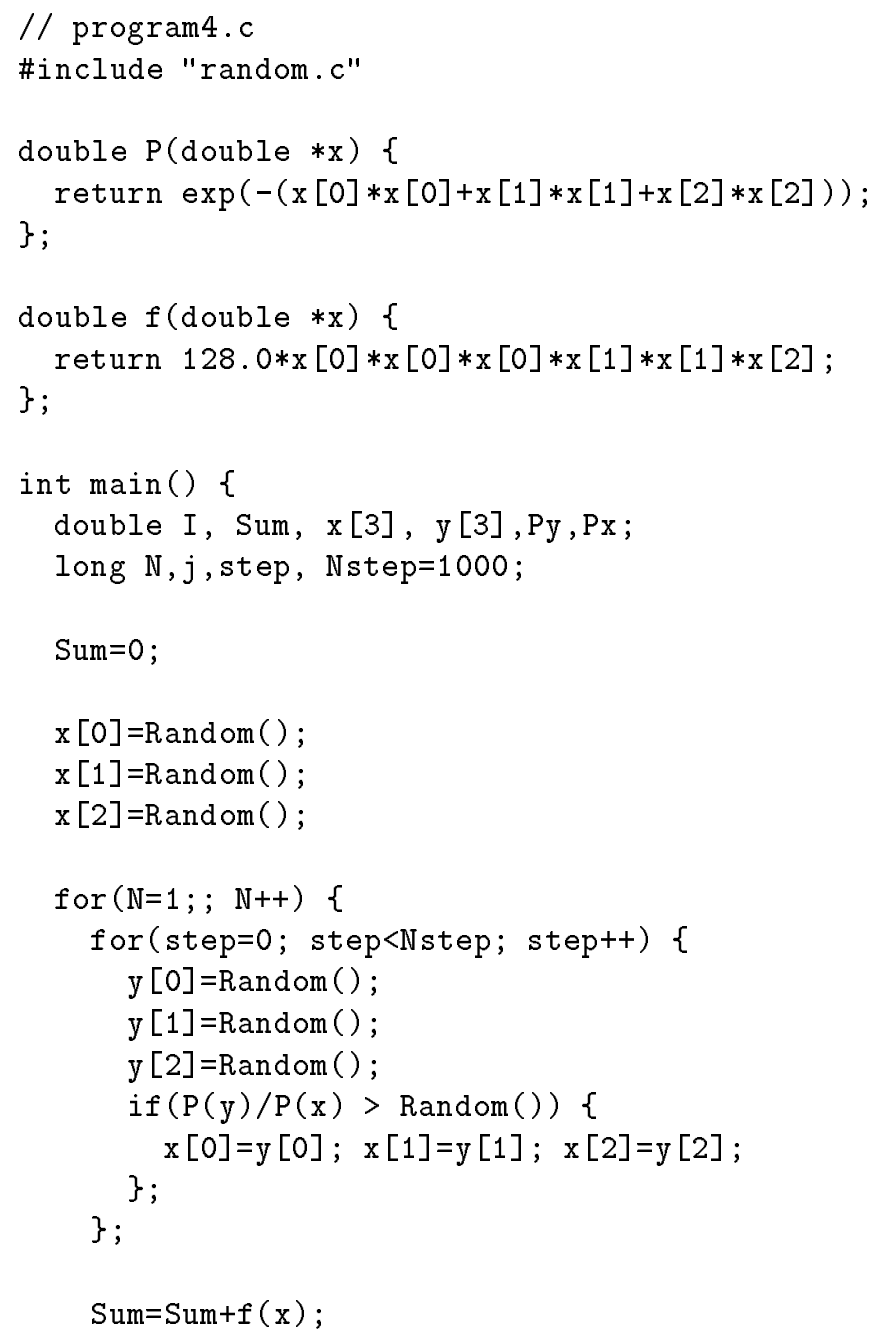




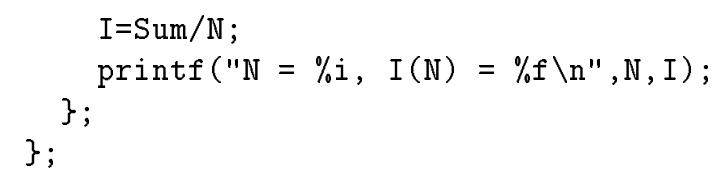

\section{B.5 Metropolis and Bootstrap: program5.c}

// program5.c

\#include "random. c"

$\begin{array}{ll}\text { long } \mathrm{D}=3 ; & / / \text { number of dimensions } \\ \text { long } \mathrm{N}=8192 ; & / / \text { number of Montecarlo samples } \\ \text { long } M=100 ; & / / \text { number of Bootstrap samples }\end{array}$

long Nstep=1000;// number of interations per config.

double $P($ double $* x)\{$

return $\exp (-(\mathrm{x}[0] * \mathrm{x}[0]+\mathrm{x}[1] * \mathrm{x}[1]+\mathrm{x}[2] * \mathrm{x}[2]))$;

\} ;

double $f($ double $* x)\{$

return $128.0 * \mathrm{x}[0] * \mathrm{x}[0] * \mathrm{x}[0] * \mathrm{x}[1] * \mathrm{x}[1] * \mathrm{x}[2]$;

\} ;

void swap (double \&a, double \&b) \{

double $c$;

$\mathrm{c}=\mathrm{a} ; \mathrm{a}=\mathrm{b} ; \mathrm{b}=\mathrm{c}$;

\} ;

int $\operatorname{main}()\{$

double I, Sum;

double $x[N][D]$;

double y $[D]$;

double Ibar $[M]$;

long i,j,d,k[N] [M], step;

// //////////////////////////////////

// generate confifgurations using Metropolis

$1 / / / / / / / / / / / / / / / / / / / / / / / / / / / / / / / / / / / / / / / / / /$

for $(d=0 ; d<D ; d++) \times[0][d]=\operatorname{Random}()$;

for $(i=1 ; i<\mathrm{N} ; i++)\{$

for $(d=0 ; d<D ; d++) x[i][d]=x[i-1][d]$;

for (step $=0$; step<Nstep; step++) \{

for $(\mathrm{d}=0 ; \mathrm{d}<\mathrm{D} ; \mathrm{d}++)$ y $[\mathrm{d}]=$ Random () ;

if $(\mathrm{P}(\mathrm{y}) / \mathrm{P}(\mathrm{x}[\mathrm{i}])>\operatorname{Random}())$ 


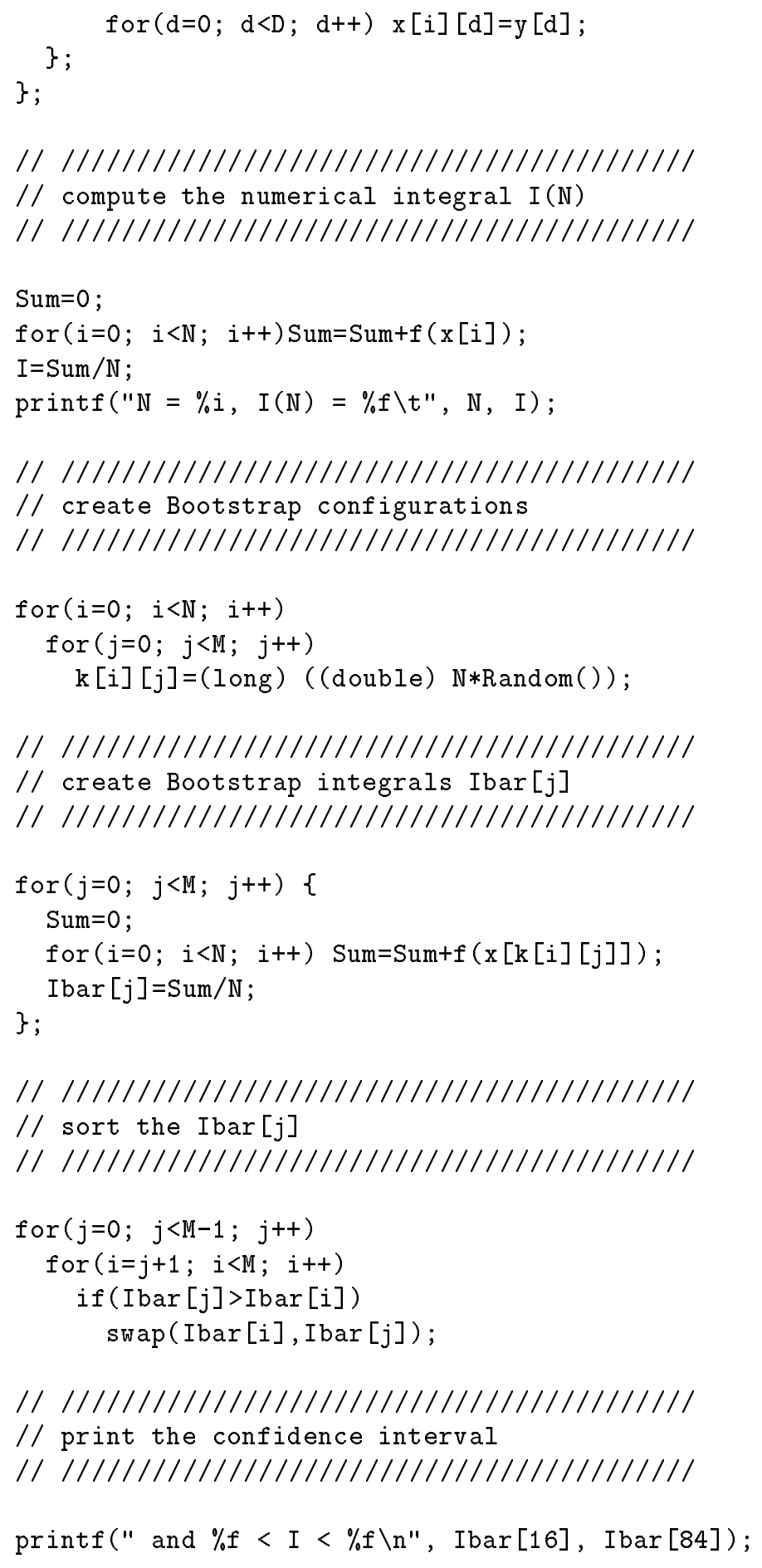




\section{B.6 Marsaglia random number generator: random.c}

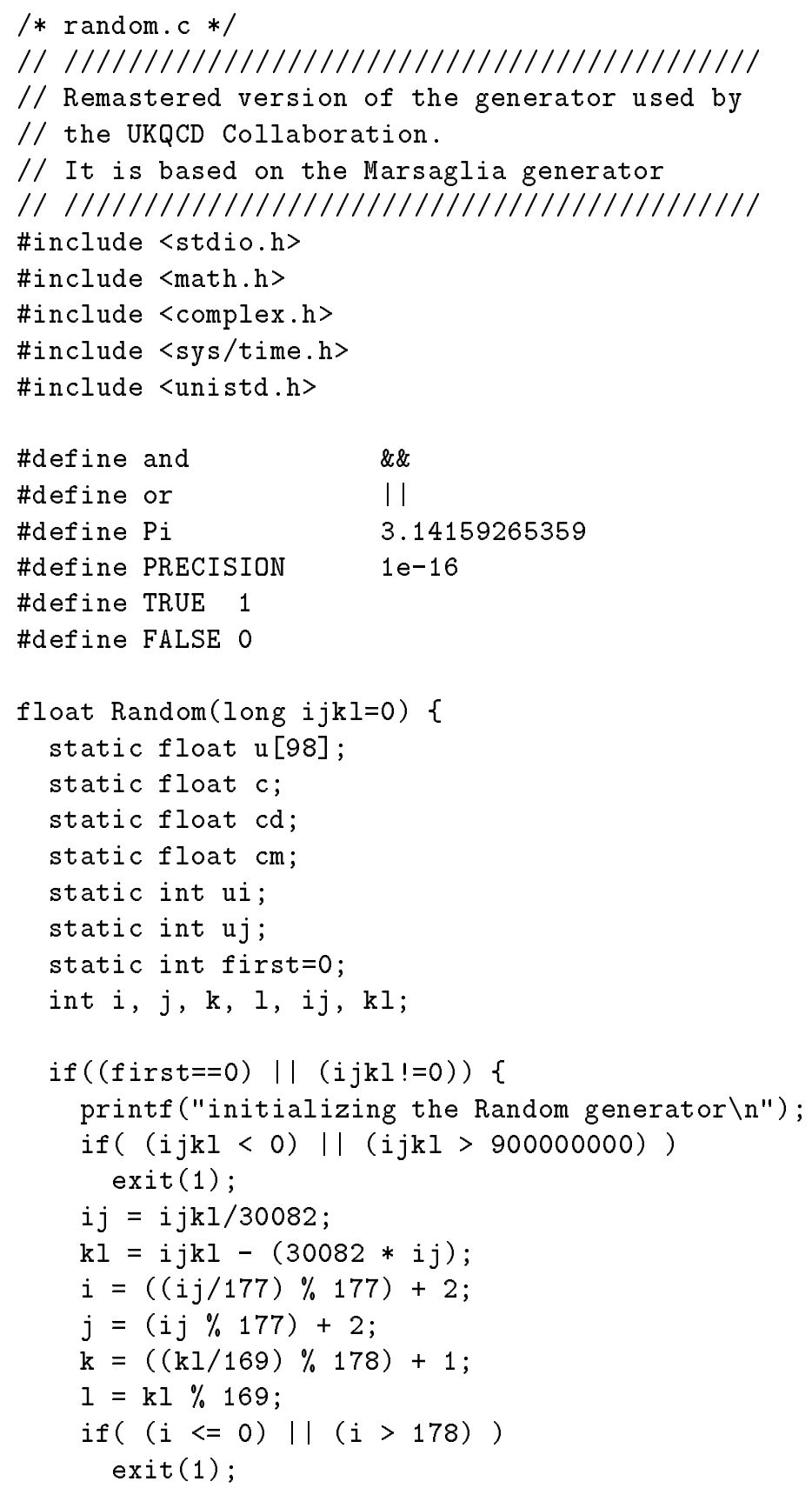




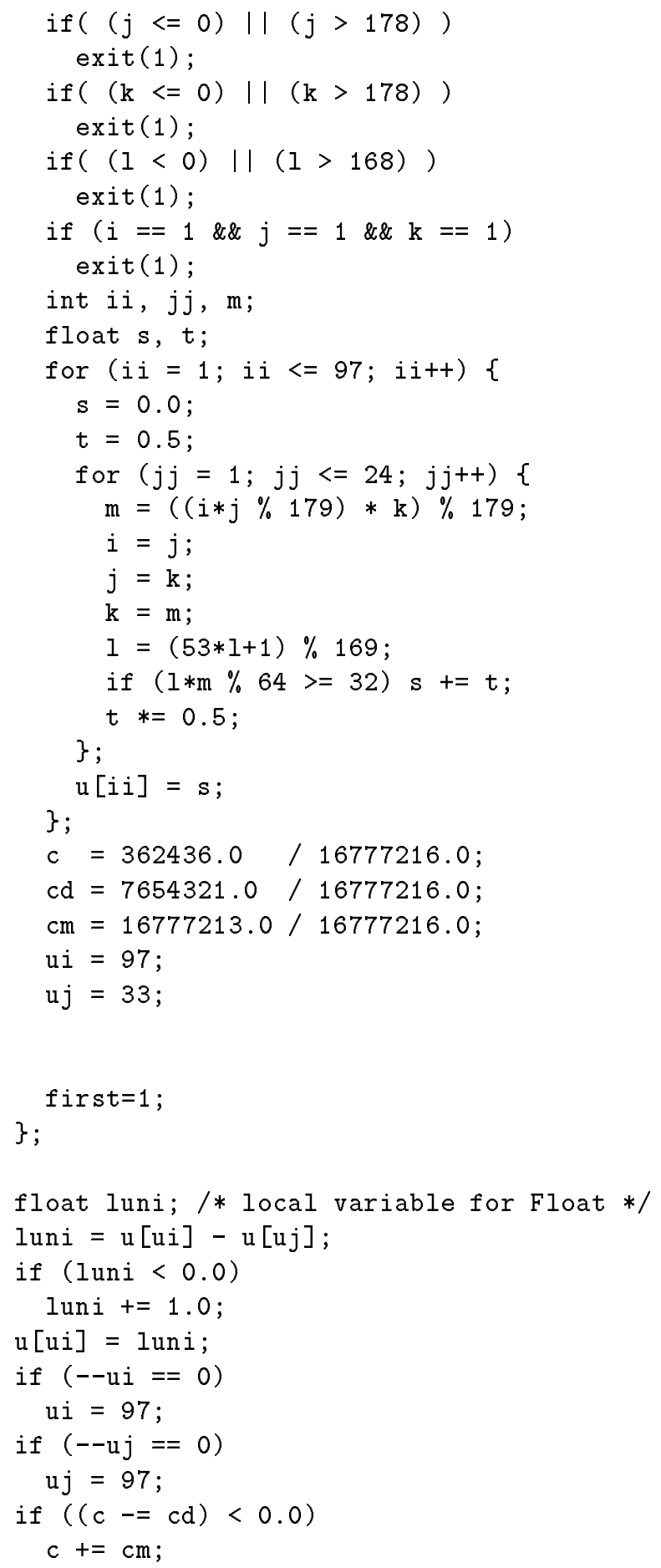




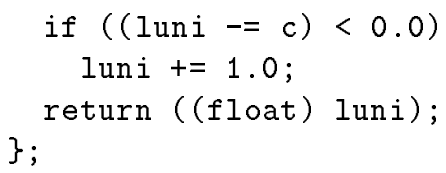

B.7 $f_{B}$ and $m_{B}$. Program: C2.C

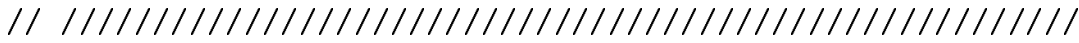

// Program C2.C written by Massimo Di Pierro O July 2000

$/ / / / / / / / / / / / / / / / / / / / / / / / / / / / / / / / / / / / / / / / / / / / / / / / / / 1$

// WORKING EXAMPLE of a Lattice QCD program to compute the

// Euclidean propagator of an Heavy-Light Meson, C2(t)

$/ /$ To extract $m_{-} B$ and $f_{-} B$ fit output with

$1 /$

$/ / C 2(t)=1 / 2 f_{-} B^{\wedge} 2 m_{-} B \exp \left(-m_{-} B t\right)+\ldots$

$1 /$

$/ /$ and extrapolate to

$/ / \mathrm{mq} \rightarrow 0$ (GeV)

$/ / \mathrm{mh} \rightarrow \mathrm{mb}$ (GeV) (the b quark pole mass)

$/ /$ a $\rightarrow 0 \quad\left(\mathrm{GeV}^{-}(-1)\right)$

// ////////////////////////////////////////////////

// \#define PARALLEL

// /////////////////////////////////////////////////

// open the libraries: Matrix Distributed Processing 1.0

$/ /$ (for a description read: hep-lat/0004007)

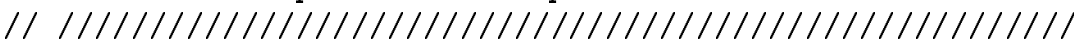

\#include "MDP_Lib2.h"

\#include "MDP_MPI.h"

$/ / / / / / / / / / / / / / / / / / / / / / / / / / / / / / / / / / / / / / / / / / / / / / / / 1$

// open the FermiQCD libraries

$/ / / / / / / / / / / / / / / / / / / / / / / / / / / / / / / / / / / / / / / / / / / / / / / / / /$ \#include "MDP_Gauge.h"

\#include "MDP_Fermi.h"

\#define $\mathrm{GeV} 1$

$/ / / / / / / / / / / / / / / / / / / / / / / / / / / / / / / / / / / / / / / / / / / / / / / /$

// main program

$/ / / / / / / / / / / / / / / / / / / / / / / / / / / / / / / / / / / / / / / / / / / / / / / /$

int main(int argc, char **argv) \{

mpi.open_wormholes(argc, argv); // open communications 


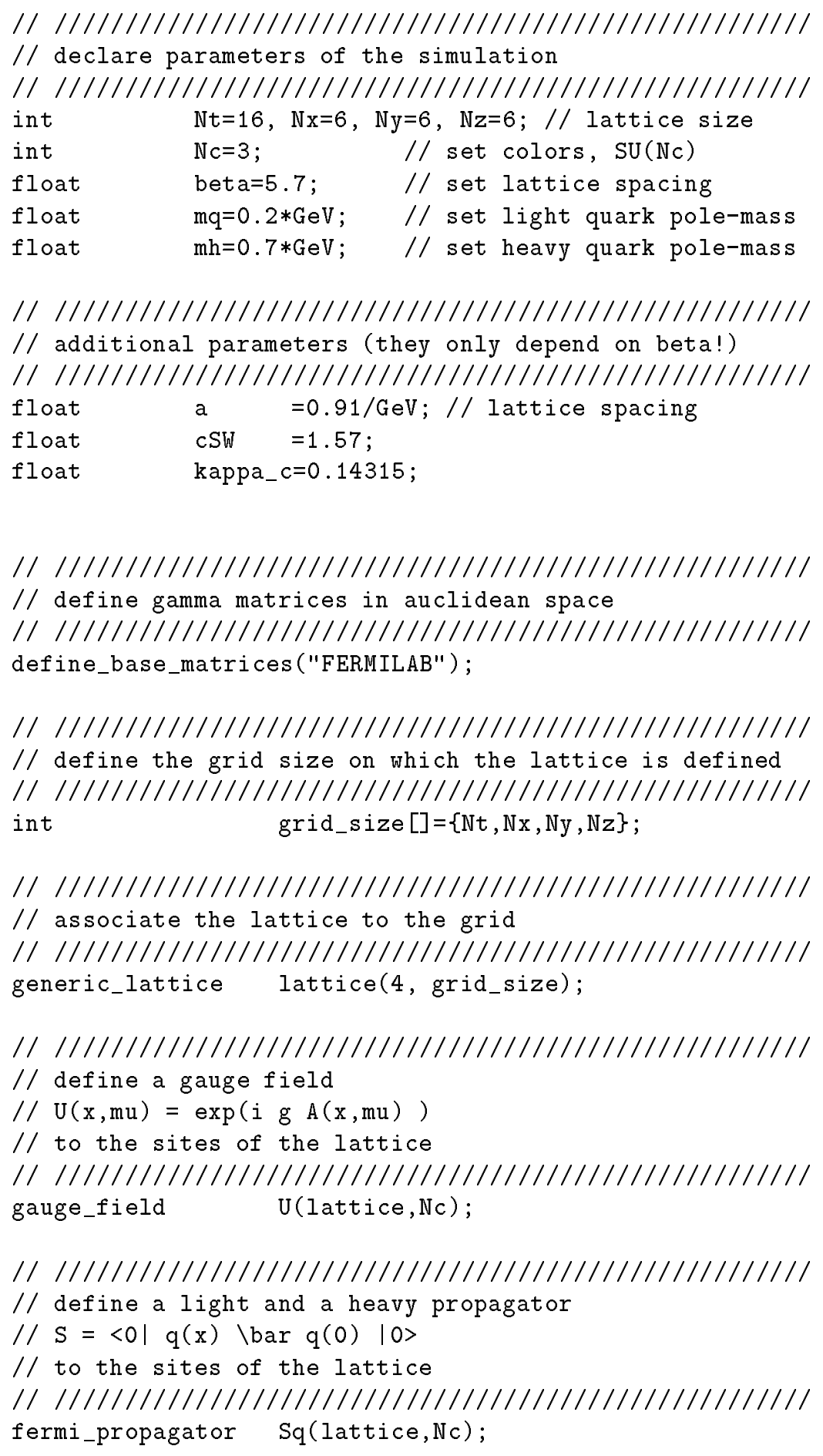




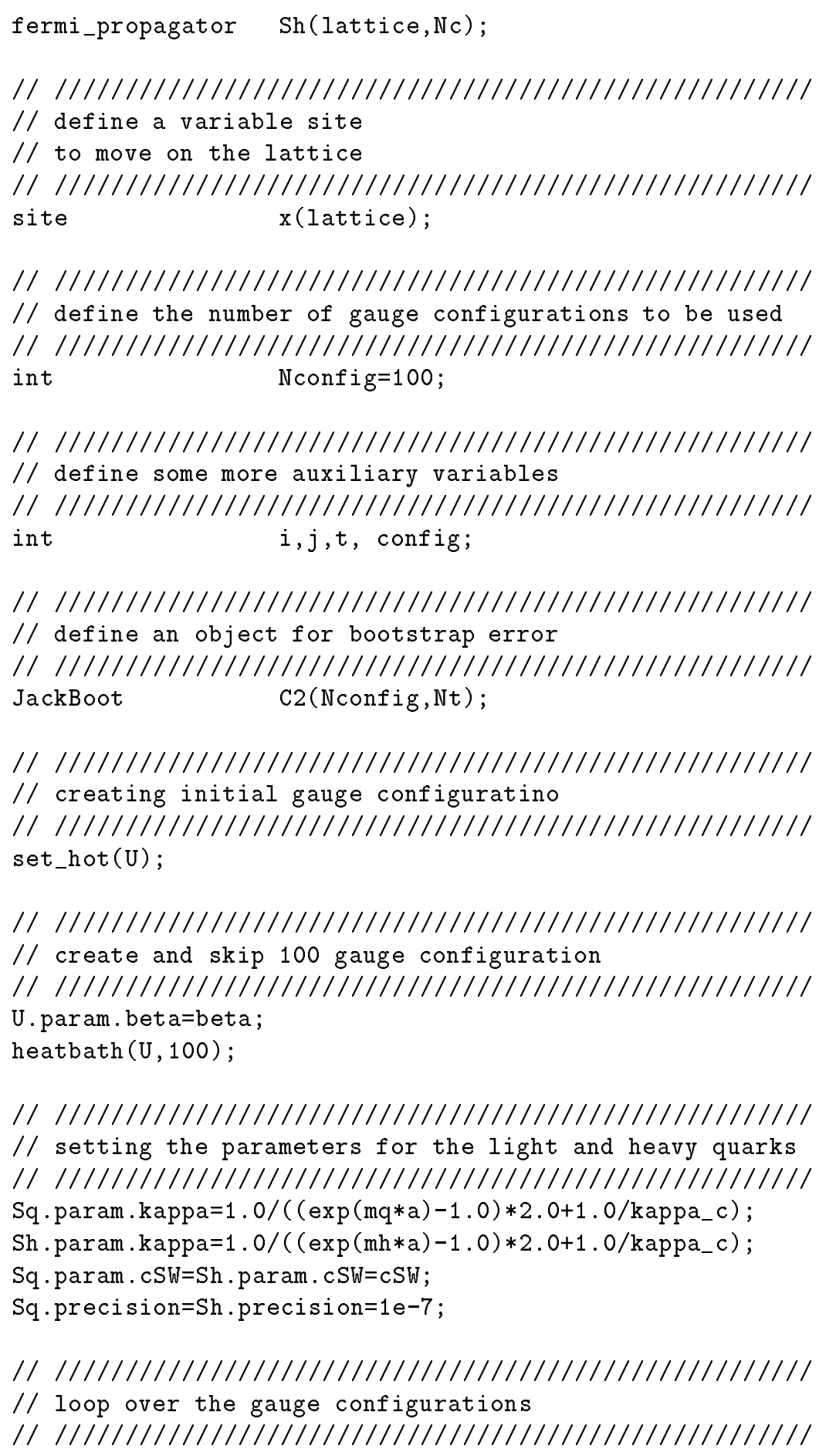




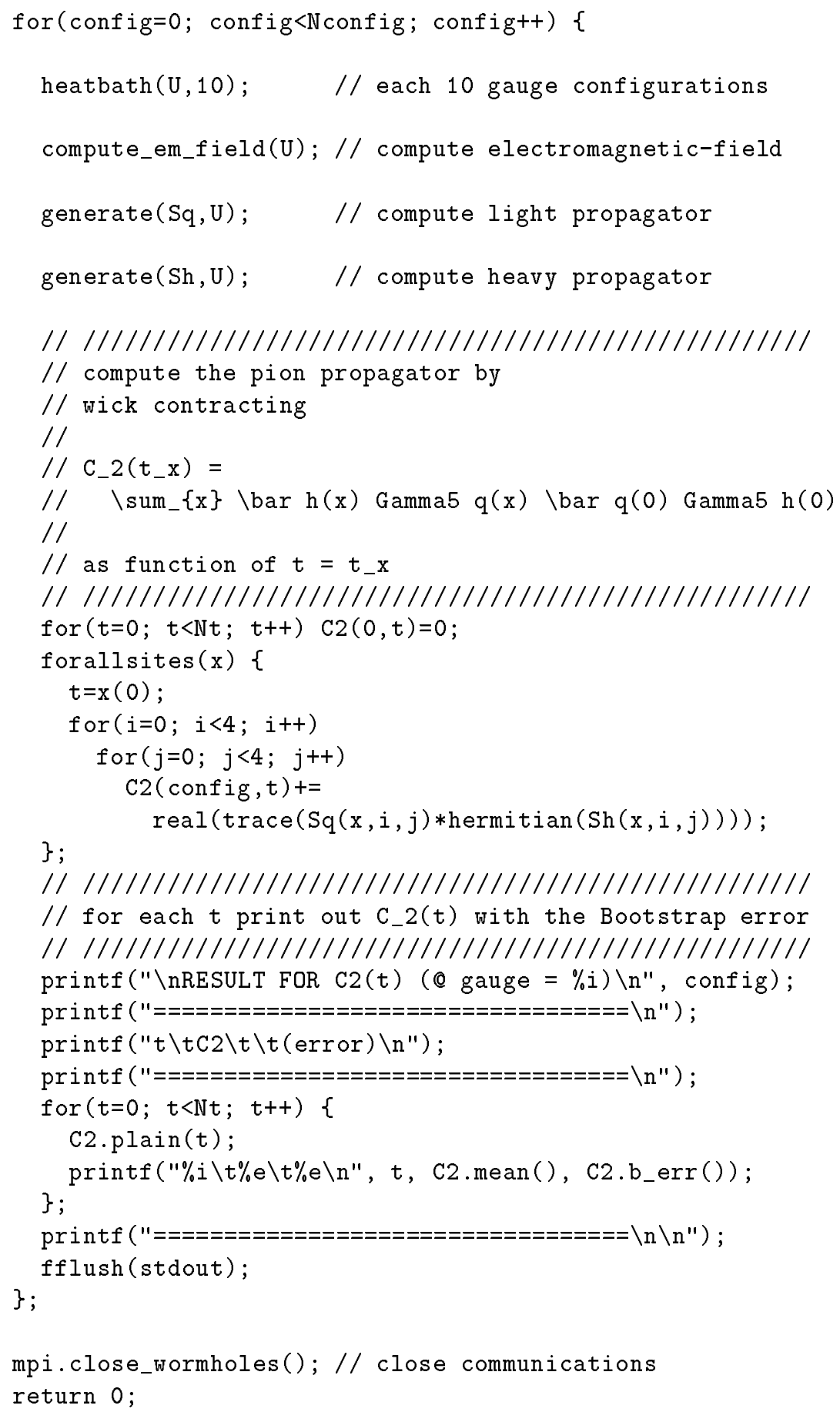




\section{Status of Lattice QCD}

In this appendix we will briefly report a very small subset of recent Lattice QCD results which have direct phenomenological interest and which provide an example of the state-of-the-art in Lattice QCD simulations ${ }_{-\ldots}^{15{ }^{\prime}}$

A huge amount of work has also been dedicated by the lattice community to the study of some theoretical and numerical aspects of field theories and the properties of their lattice regulated versions. They include the study of different possible discretizations for the Dirac operator (ID) sstudy of low energy eigenvalues of these operators $\Gamma$ the confining properties of different Yang-Mills theories and chiral symmetry breaking. These studies have given some important insights in the understanding of QCD and constitute the foundations on which any simulation of phenomenological interest relies on.

- Chiral symmetry on the Lattice. The light spectrum of QCD is dominated by spontaneous chiral symmetry breaking (in fact the pion is both a bound state and a Goldstone boson). At a classical level the continuum Dirac operator ГДD Гpreserves the chiral symmetry $\Gamma$ which we rewite as the Ginsparg-Wilson relation

$$
\gamma^{5} \not D+\not D \gamma^{5}=0
$$

The chiral symmetry is broken $\Gamma$ at the quantum level $\Gamma$ by the chiral anomaly.

The lattice regularized Dirac operator $\Gamma$ eq. $\left(\underline{\overline{6}} \underline{6}_{1}^{\prime}\right)$ Texplicitely breaks this symmetry and therefore does not provide a satisfatory description of chiral physics.

Since today no-one succeded in writing down a discretized versions of chiral fermions because of the famous Nielsen-Ninomiya no-go theorem [3-13]. Recently two solutions have been found to this problem and they are both equivalent to modify the chirality condition $\Gamma$ eq $\left(\begin{array}{l}1 \\ 2\end{array} \overline{3}-1\right) \Gamma$ into

$$
\gamma^{5} \not D+\not D \gamma^{5}=a \not D \gamma^{5} \not D
$$

where the right-hand side vanishes in the limit $a \rightarrow 0$.

\footnotetext{
${ }^{15}$ The papers quoted below are chosen as examples and we do not aim to provide a complete list of references on any of the subjects.
} 


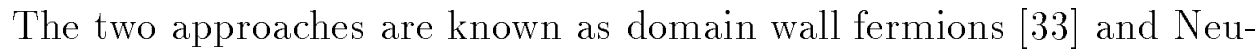
berger fermions $\left[\begin{array}{l}34 \\ 4\end{array}\right.$

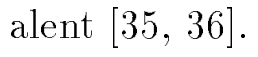

The work in this subject has still to be considered exploratory but seems very promising. Moreover it provides a theoretical testing ground for other well-estabilished lattice discretizations of fermions $\Gamma$ such as Sheikoleslami-Wolhert (also known as Clover) and Kogut-Susskind (also known as Staggered) fermions.

At present the most significant phenomenological lattice results are computed using Clover (and Staggered) fermions. Here "significant" means that simulations with Clover (and Staggered) fermions have been performed in a wide range of lattice spacings $\left(1 \mathrm{GeV}<a^{-1}<3 \mathrm{GeV}\right) \Gamma$ on relatively large lattices (up to $48 \times 24^{3}$ and even bigger) and for many different values of the light quark masses. Large statistical Monte Carlo samples (of the order of hundreds of configurations) have been created and scrutinized.

Moreover the most important results of phenomenological interest have been reproduced indipendently by different international collaborations and agree with each other within the statistical errors.

- Light hadronic spectrum. Fig. 121 shows some of the most recent lattice results for the mass specrtum of light mesons and baryons $\Gamma$ as computed by the CP-PACS collaborations. These results are obtained using Clover fermions Textrapolated to the chiral limit Tin the quenched approximation. The error includes the effect of the chiral extrapolation but does not include the unknown effect of quenching.

Fig. 1 13 shows a comparison between quenched and unquenched results for the mass of light hadrons as function of the lattice spacing. Quenched results are easier to compute therefore have a much smaller statistical error. At present their error is dominated by the systematic one. The comparison with unquenched results can be used to quantify this systematic error.

- Glueball spectrum. One sector in which lattice calculations have absolutely no competitors (because no models are available) is the computation of the glueball spectrum. Fig. ilisis shows some lattice results 
obtained in ref. [3] experiments.

- Confinement. Lattice has been the first theoretical tool to give direct evidence of the phenomenon of confinement. This is done through the computation of the Wilson loop Tfrom which one obtains the chromoelectro-magnetic potential between a couple of static quarks-antiquark. This potential (both for quenched and unqueched simulations) is shown in fig. quenched approximation $\Gamma$ grows linearly because no new particles can appear in the vacuum to screen the two static particles. The linearity is explained with the dual-superducting property of the QCD vacuum $\left[\begin{array}{l}\overline{1} \\ \overline{0}\end{array}\right]$. It forces the chromoelectric field of the system of two particles to be squeezed into a flux tube connecting them: the string. In the unquenched (full) theory ${ }^{2}$ instead $\Gamma$ this potential only grows up to the point when the string contains enough potential energy to create in the QCD vacuum a new quark-antiquark pair that breaks the string itself. Hence the potential reaches a plateau in correspondence to the treshold energy.

The deviation between the quenched and the unquenched potential has not been observed so far because $\Gamma$ for numerical reasons $\Gamma$ dynamical quark masses are still too heavy and lattice volumes too small.

The computation of the chromo-electromagnetic potential $\Gamma$ combined with experimental measurements provides the best present determination of $\alpha_{s}$ (fig. $1 \overline{0}$ ) Twhich is the expansion parameter of any perturbative QCD calculation.

- Matrix elements and decays. Almost all decays of hadrons can be parametrized in terms of matrix elements that encode the nonperturbative contribution of QCD. For many of these matrix elements lattice computations have been able to produce satisfactory results. In table ${ }_{2}^{5}$ we list $\Gamma$ as an example $\Gamma$ some of those matrix elements. Some other results are not conclusive and occasionally very controversial (for example attempts to compute $\epsilon^{\prime} / \epsilon$ ).

Matrix elements that include a contribution of final state interactions (for example $B \rightarrow \pi \pi$ ) have so far been outside the reach of lattice

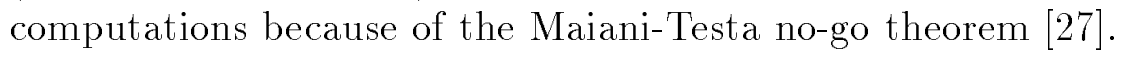




\begin{tabular}{|l|l|l|}
\hline Matrix element & Process & Parameters \\
\hline$\left\langle 0\left|b \gamma^{\mu} q\right| B\right\rangle \equiv f_{B} p^{\mu}$ & $B \rightarrow$ leptons & $\left|V_{t d}\right| \Gamma\left|V_{t s}\right|$ \\
$\left\langle 0\left|\bar{c} \gamma^{\mu} q\right| D\right\rangle \equiv f_{D} p^{\mu}$ & $D \rightarrow$ leptons & \\
$\langle D|\bar{c} \Gamma s| K\rangle$ & $D \rightarrow K+$ leptons & $\left|V_{c s}\right|$ \\
$\left\langle K|\bar{s} \Gamma u| M_{u}\right\rangle$ & $K \rightarrow M_{u}+$ leptons & $\left|V_{u s}\right|$ \\
$\left\langle D|\bar{c} \Gamma d| M_{d}\right\rangle$ & $D \rightarrow M_{d}+$ leptons & $\left|V_{c d}\right|$ \\
$\left\langle B|\bar{b} \Gamma u| M_{u}\right\rangle$ & $B \rightarrow M_{u}+$ leptons & $\left|V_{u b}\right|$ \\
$\left\langle\bar{B}|\bar{b} \Gamma c| D^{*}\right\rangle$ & $\left|V_{c b}\right|$ \\
$\left\langle B|\bar{q} \Gamma q| B^{*}\right\rangle$ & $\left|V_{u b}\right|$ \\
$\langle K|\bar{s} \Gamma q \bar{s} \Gamma q| \bar{K}\rangle \propto B_{K}$ & $(K \rightarrow \pi+$ leptons & $\left|V_{t d}\right| \Gamma\left|V_{t s}\right|$ \\
$\langle D|\bar{c} \Gamma q \bar{c} \Gamma q| \bar{D}\rangle \propto B_{D}$ mixing $)$ & $(D-\bar{D}$ mixing $)$ & $\left|V_{t d}\right| \Gamma\left|V_{t s}\right|$ \\
$\langle B|\bar{b} \Gamma q \bar{b} \Gamma q| \bar{B}\rangle \propto B_{B}$ & $(B-\bar{B}$ mixing) & \\
$\langle B|\bar{b} \Gamma b| B\rangle$ & $B$ kinetic and magnetic energy & \\
$\langle B|\bar{b} \Gamma q \bar{q} \Gamma b| B\rangle$ & inclusive $B$ decay & \\
$\left\langle\Lambda_{b}|\bar{b} \Gamma q \bar{q} \Gamma b| \Lambda_{b}\right\rangle$ & inclusive $\Lambda_{b}$ decay & \\
\hline \multicolumn{2}{|l}{}
\end{tabular}

Table 2: Examples of matrix elements usually computed on the lattice and related processes $\left(M_{q}\right.$ stands for the most general $\left(q q^{\prime}\right)$ meson $\Gamma$ for example $\pi \Gamma \rho$ or $K ; \Gamma$ is the most general spin $\otimes$ color matrix). The table also shows the $V_{C K M}$ matrix elements that are associated to the processes. 
- Lattice, effective field theories and models. Since lattice can be used to compute matrix elements and these can be confronted with predictions from effective theories or models Tit becomes possible to use the lattice results to extract their effective parameters. As an example in ref. $[\overline{4} 2]$ the effective coupling of the Heavy Meson Chiral Lagrangian is measured on the lattice using a numerical computation for the following matrix element

$$
E(r)=\frac{1}{3} \sum_{\mu=1,2,3} \int\left\langle B\left|A_{\mu}(\mathrm{x})\right| B^{*}\right\rangle \mathrm{d} \Omega_{\mathrm{x}}
$$

where $A_{\mu}(x)$ is the axial current and $\Omega_{\mathrm{x}}$ is the solid angle associated to the $3 \mathrm{D}$ vector $\mathrm{x}$. Fig. $\bar{I}_{1} \overline{\mathrm{T}}$ shows a comparison between the lattice result for $E(r)$ and a prediction of the Chiral Quark Model. The parameters of the model have been adjusted to fit the experimental mass spec-

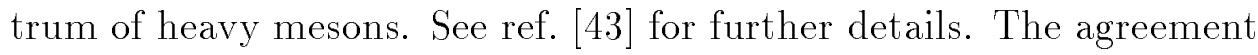
between the lattice (a first principle simulation) and the model (consequence of experimental observations and some theoretical assumptions) is remarkable.

- Heavy quarks and CKM Matrix. As we have shown in Section 1 the Cabibbo-Kobayashi-Maskawa matrix elements have to be extracted from a comparison between experimental and theoretical predictions. The latter $\Gamma a t$ present $\Gamma$ are computed on the lattice with a non-negligible uncertainty. Table $\underset{2}{2}$ includes a list of those processes that mainly contribute to the determination of the CKM matrix elements and have to be computed using lattice simulations.

Fig. I $\overline{8}_{1}($ top) shows the present constraints on the CKM mixing angle in the $(\bar{\rho} \Gamma \bar{\eta})$ plane (where $\rho$ and $\eta$ are parameters of the Wolfenstein parametrization of the CKM matrix) and should be compared with fig. il (bottom) which is obtained using the same experimental and theoretical input but assuming a possible future uncertainty instead of the "real" present one for the lattice parameters. The future uncertainty is based on being able to generate 1000 gauge configurations with a lattice spacing of $a=0.08 \mathrm{fm}$ and with $m_{\pi} / m_{\rho}=0.4[\overline{4} \overline{1}]$ (the latter constraint measures how well one is approaching the chiral limit). This estimate also assumes that systematic error due to quenching are under control. 
The comparison shows how important it is to invest in lattice simulations while $\Gamma$ contemporary investing in experimental facilities. In fact for many fundamental quantities the theoretical uncertainty is as significant (if not more) than the experimental one. 


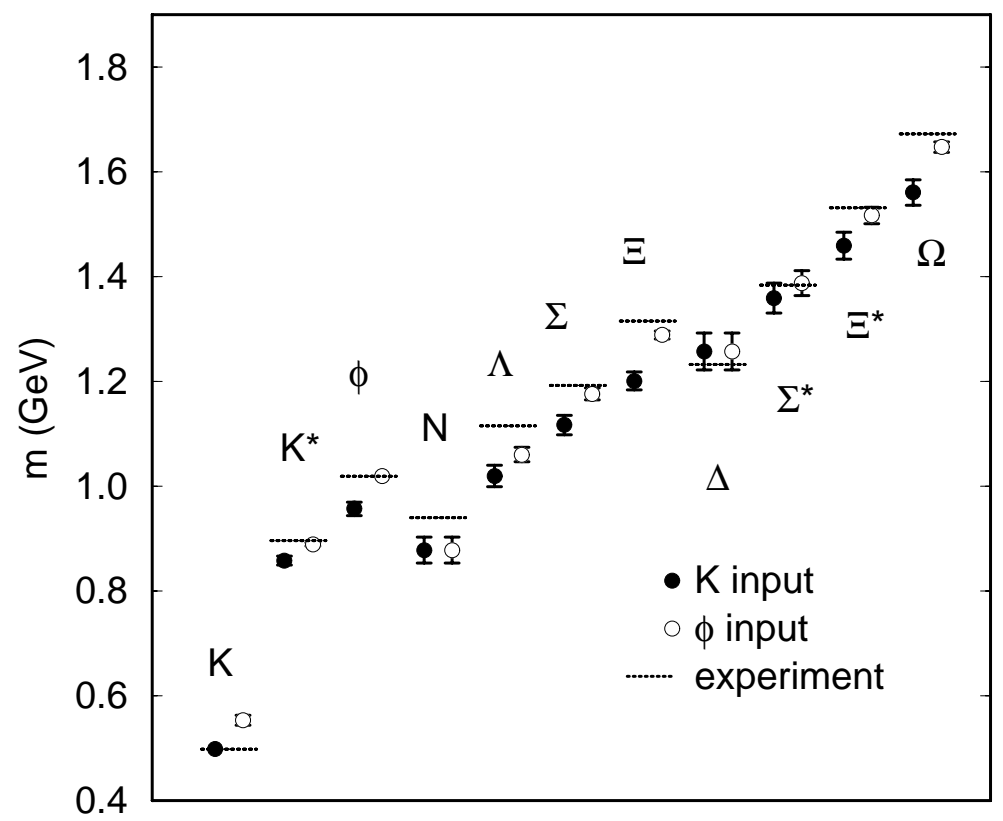

Figure 12: Light hadrons spectrum as computed by the CP-PACS collabo-

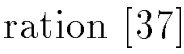




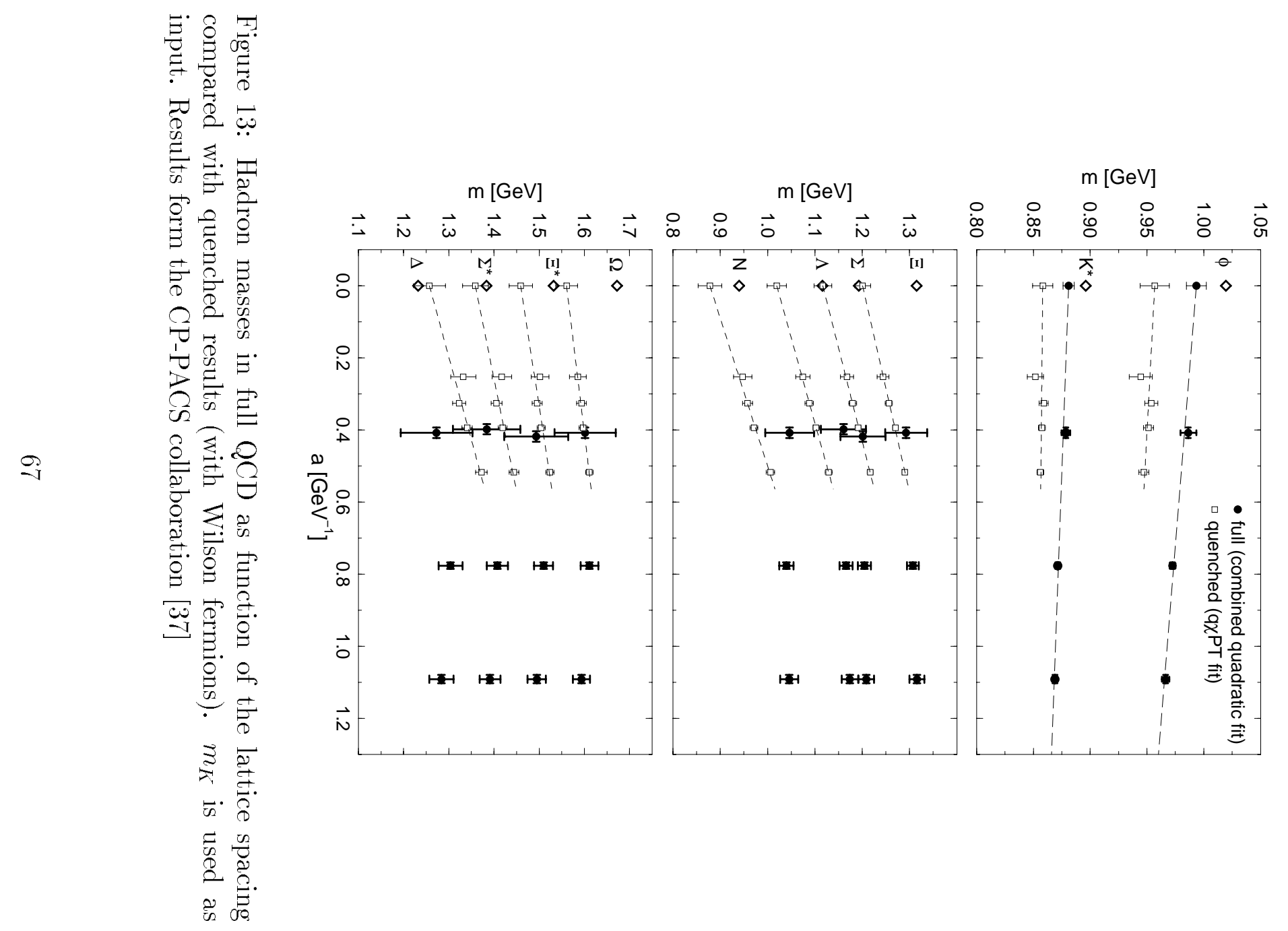




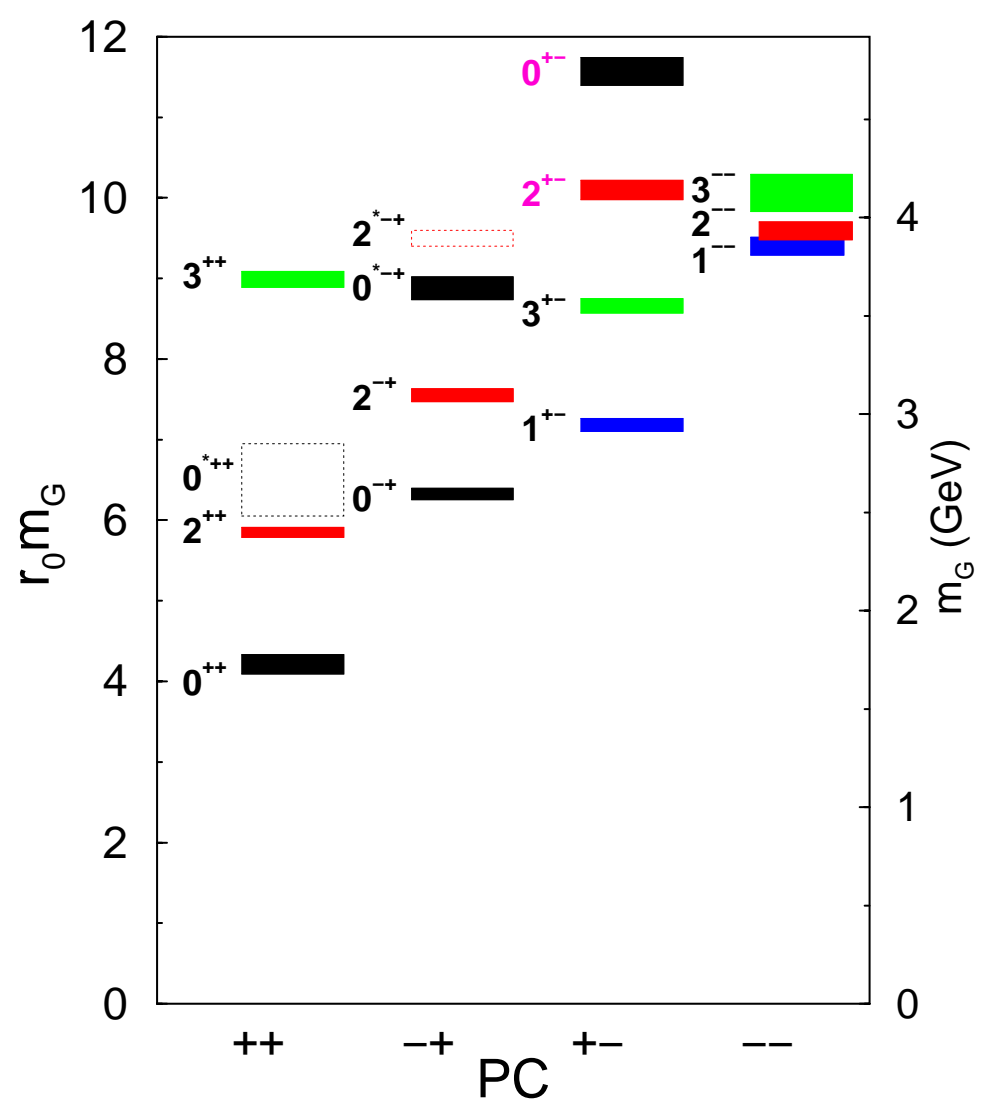

Figure 14: Mass spectrum in the SU(3) quenched theory as computed in

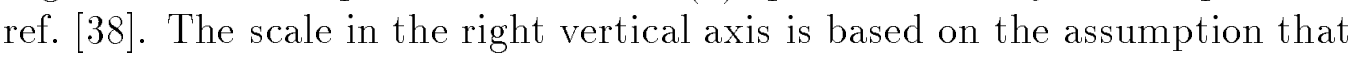
$r_{0}=410 \mathrm{MeV}$. 


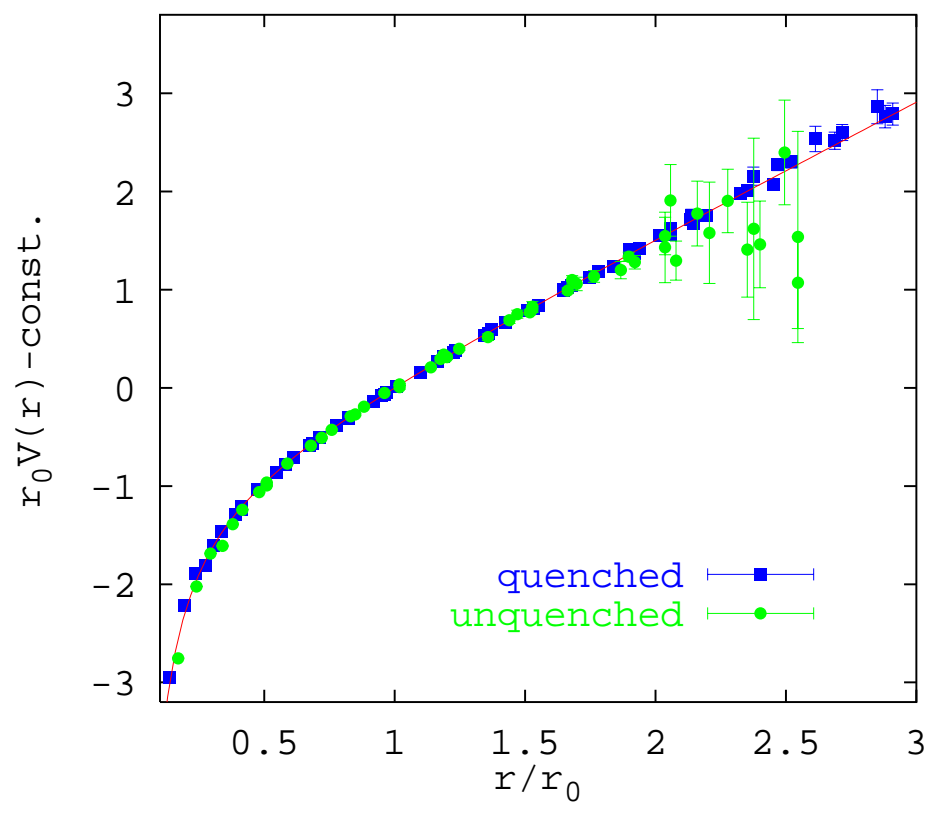

Figure 15: String tension in quenched and unquenched (two dynamical light flavours at $\kappa=0.1575$ ) Textracted from ref. [39 $\overline{9}$ 


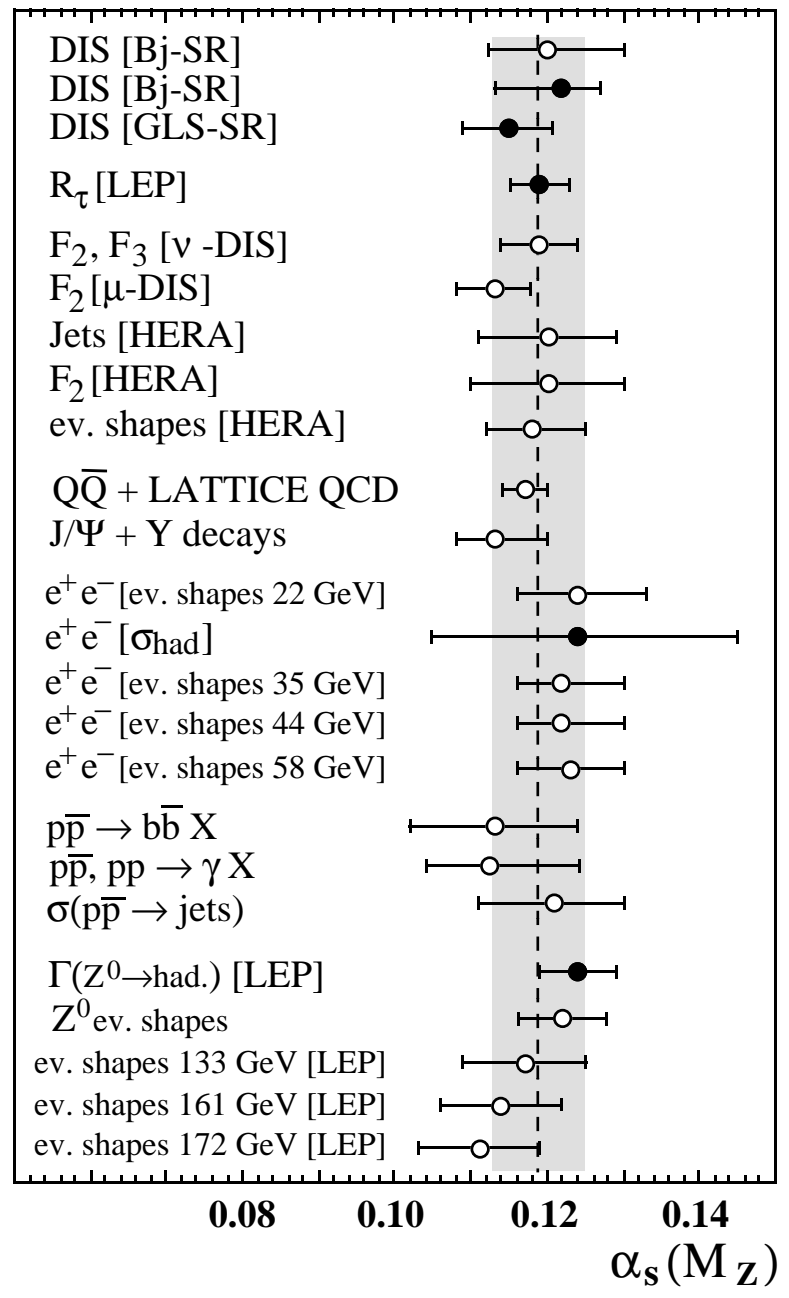

Figure 16: Comparison among different determinations for $\alpha_{s}$ at the $Z$ pole mass. 


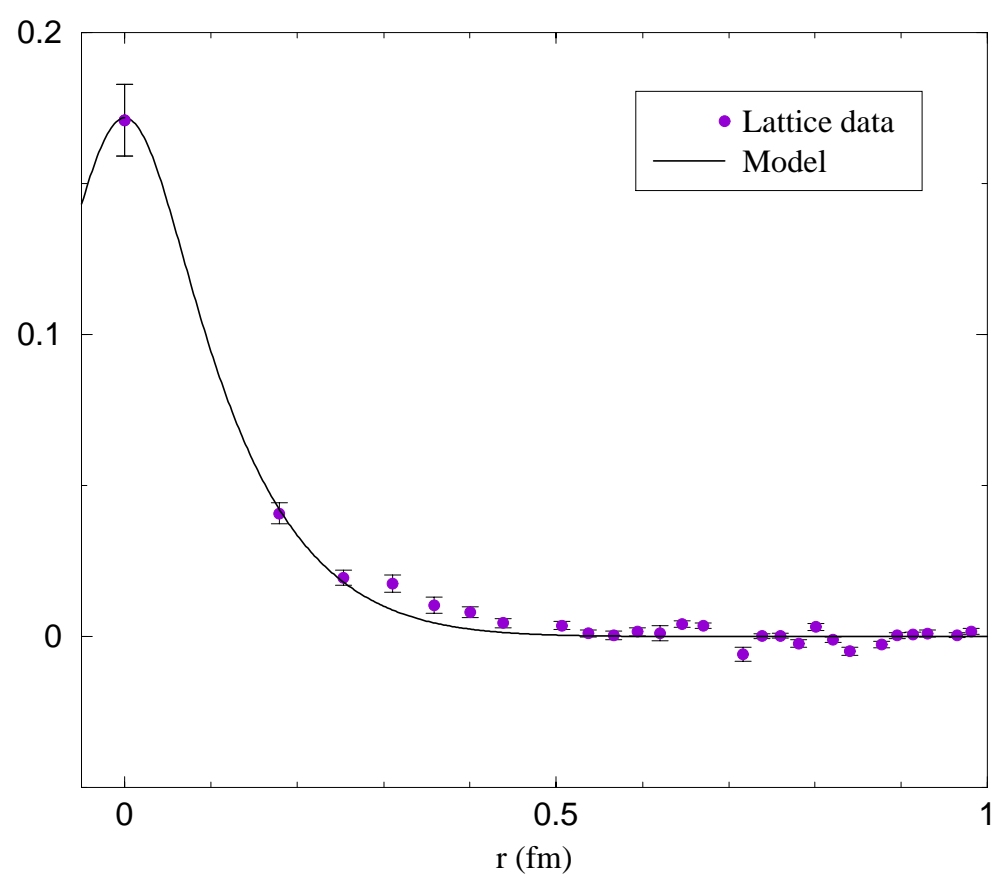

Figure 17: Comparison of a Lattice QCD matrix element $\Gamma E(r) \Gamma$ and the same matrix element evaluated in the Chiral Quark Model. 

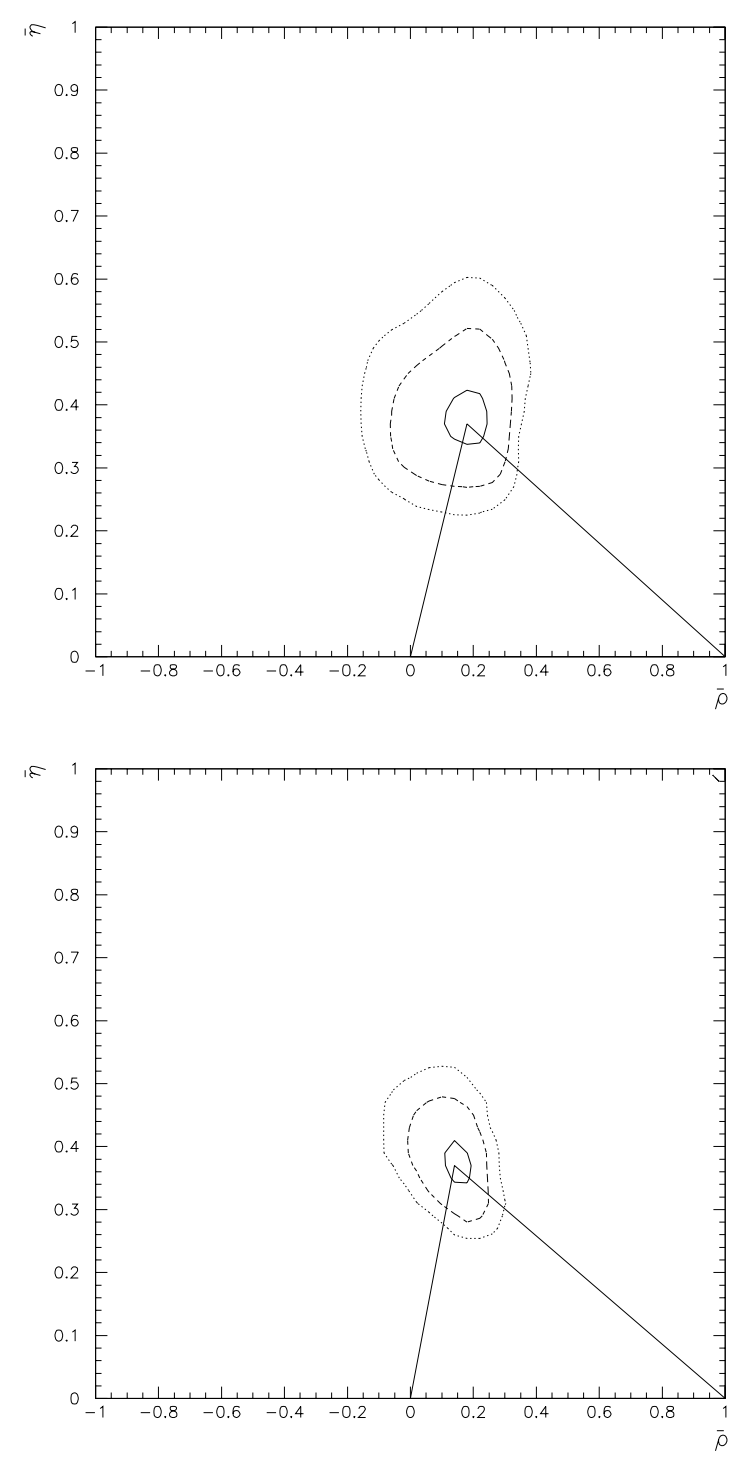

Figure 18: Current (top) and future (bottom) allowed regions for the point $(\bar{\rho} \Gamma \bar{\eta}) \Gamma$ the vertex of the unitarity triangle. The three regions correspond to $5 \%$ Г $68 \%$ and $95 \%$ confidence levels. The future estimate is based on the

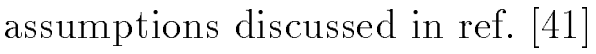




\section{References}

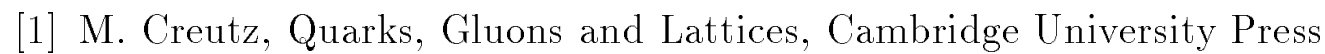
(1983)

[2] RotheCLattice Gauge TheoriesTWorld Scientific

[3] I. Montvay and G. MünsterTQuantum Fields on a LatticeГCambridge University Press (1994)

[4] M. Peskin and D.V. ShroederTQuantum Field TheoryГAddison Wesley (1994)

[5] T. Muta TFoundations of Quantum Chromo Dynamics WWorld Scientific (1987)

[6] W. Marciano and H Pagels TPhys. Rep. 36 (1978) 135

[7] D. J. Gross and F. WilczekTPhys. Rev. Lett. 30 (1973) 1343

D. J. Gross and F. WilczekГPhys. Rev. D8 (1973) 3633

H. PolitzerГPhys. Rev. Lett. 30 (1973) 1346

H. PolitzerTPhys. Rep. 14C (1974) 129

[8] G. 't Hooft TPhys. Rev. (1978) 1

[9] J. F. DonoghueГE. Golowich and B. Holstein DDynamics of the Standard Model Cambridge University Press (1996)

[10] N. CabibboГPhys Rev. 10 (1963) 1802

J. Kobayashi and M. Maskawa Prog. Theor. Phys. 49 (1973) 952

[11] G. BanhotTRep. Prog. Phys. 51 (1988) 429

[12] J. Shao and D. TuГ The Jackknife and Bootstrap $\Gamma$ Springer Verlag (1995)

[13] J. M. Rabin Introduction to Quantum Field Theory for Mathematicians ГIAS/Park City Mathematics Series Tvol. 1 (1995)

[14] J. Glimm and R. JaffeГ Quantum Physics (second edition) TSpringerVerlag (1987).

[15] G. 't Hooft and M. Veltman NNucl. Phys. B44 (1972) 189 
[16] L. D. Faddeev and V. N. Popov PPhys. Lett. B25 (1967) 29

[17] G. P. LepageГnucl-th/970629

[18] S. Weinberg Thep-th/9702027

[19] T. Applequist and J. CarazzoneГPhys.Rev. D11 (1975) 2856

[20] K. Symanzik TNucl. Phys. B226 (1983) 187205

[21] M. CreutzTQuantum Fields on the Computer TWorld Scientific (1992)

[22] K. G. WilsonГPhys. rev. D10 (1974) 2445

[23] B. Sheikoleslami and R. WolhertTNucl. Phys. B259 (1985) 572

[24] M. Luscher $\Gamma$ Advanced Lattice QCD Talk given at Les Houches Summer School in Theoretical PhysicsTSession 68Г(1998); hep-lat/9802029

[25] N. Cabibbo and E. MarinariГPhys. Lett. 119B (1982) 387

[26] C. T. SachrajdarLattice Simulations and Effective Theories TLectures presented at the Advanced School on Effective Theories $\Gamma$ Almunecar (Spain) June 1995; hep-lat/960527

[27] L. Maiani and M. TestaГPhys.Lett. B245 (1990) 585-590

[28] A. X. El-KhadraГA. S. Kronfeld and P. B. MackenzieThep-lat/9604004 $(1996)$

[29] A. X. El-KhadraГA. S. KronfeldГP. B. MackenzieГS. M. Ryan and J. N. Simone PPhys. Rev. D58 (1998) 014506

[30] M. Di PierroThep-lat/0004007; download software from: http://home.dencity.com/massimo_dipierro/mdp.html

[31] C. Bernard in "From Action to Answers" $\Gamma$ Proceedings of the 1989 TASI in Elementary Particle PhysicsTBoulderTWorld Scientific

[32] H. B. Nielsen and M. Ninomiya PPhys. Lett. B105 (1981) 219

[33] D. B. KaplanГPhys. Lett. B288 (1002) 342 
[34] R. Narayanan and H. NeubergerTPhys. Rev. Lett. 71 (193) 3251; Nucl. Phys. B41 2 (1994) 574; Nucl. Phys. B443 (1995) 305

[35] Y. ShamirTNucl. Phys. B406 (1993) 90

[36] V. Furman and Y. ShamirTNucl. Phys. B439 (1995) 54

[37] R. BurkhelterTNucl. Phys. Proc. Suppl. 73 (1999) 3

[38] C. Morningstar and M. PeardonIPhys.Rev. D60 (1999) 034509

[39] G. BaliThep-ph/9809351

[40] A. Di GiacomoГPhys.Rev. D61 (2000) 034503

[41] C. T. SachrajdaГNucl. Phys. Proc. Suppl. 83 (2000) 93

[42] G. De Divitiis et al.ГJHEP 9810 (1998) 010

[43] M. Di Pierro and E. Eichten ГProceedings of the IV International Conference on Hyperions $\Gamma$ Charm and Beauty Hadron $\Gamma$ Valcenia $\Gamma$ Spain (2000) 